(n)

Noberef tuluer

Journal of Nonlinear Mathematical Physics

ISSN (Online): 1776-0852 ISSN (Print): 1402-9251

Journal Home Page: https://www.atlantis-press.com/journals/jnmp

\title{
Semi-discrete integrable nonlinear Schrödinger system with background-controlled inter-site resonant coupling
}

Oleksiy O. Vakhnenko

To cite this article: Oleksiy O. Vakhnenko (2017) Semi-discrete integrable nonlinear Schrödinger system with background-controlled inter-site resonant coupling, Journal of Nonlinear Mathematical Physics 24:2, 250-302, DOI:

https://doi.org/10.1080/14029251.2017.1316011

To link to this article: https://doi.org/10.1080/14029251.2017.1316011

Published online: 04 January 2021 


\title{
Review ArticLe
}

\section{Semi-discrete integrable nonlinear Schrödinger system with background-controlled inter-site resonant coupling}

\author{
Oleksiy O. Vakhnenko \\ Department for Theory of Nonlinear Processes in Condensed Matter \\ Bogolyubov Institute for Theoretical Physics \\ 14-B Metrologichna Street, Kyïv, 03680-UA, Ukraine \\ vakhnenko@bitp.kiev.ua
}

Received 20 December 2016

Accepted 14 March 2017

\begin{abstract}
We summarize the most featured items characterizing the semi-discrete nonlinear Schrödinger system with background-controlled inter-site resonant coupling. The system is shown to be integrable in the Lax sense that make it possible to obtain its soliton solutions in the framework of properly parameterized dressing procedure based on the Darboux transformation. On the other hand the system integrability inspires an infinite hierarchy of local conservation laws some of which were found explicitly in the framework of generalized recursive approach. The system consists of two basic dynamic subsystems and one concomitant subsystem and it permits the Hamiltonian formulation accompanied by the highly nonstandard Poisson structure. The nonzero background level of concomitant fields mediates the appearance of an additional type of inter-site resonant coupling and as a consequence it establishes the triangular-lattice-ribbon spatial arrangement of location sites for the basic field excitations. Adjusting the background parameter we are able to switch over the system dynamics between two essentially different regimes separated by the critical point. The system criticality against the background parameter is manifested both indirectly by the auxiliary linear spectral problem and directly by the nonlinear dynamical equations themselves. The physical implications of system criticality become evident after the rather sophisticated canonization procedure of basic field variables. There are two variants of system standardization equal in their rights. Each variant is realizable in the form of two nonequivalent canonical subsystems. The broken symmetry between canonical subsystems gives rise to the crossover effect in the nature of excited states. Thus in the under-critical region the system support the bright excitations in both subsystems, while in the over-critical region one of subsystems converts into the subsystem of dark excitations.
\end{abstract}

Keywords: nonlinear lattice; integrable system; soliton; conservation laws; symmetry breaking; canonical field variables

PACS: 11.10.Lm, 45.05.+x, 02.30.Ik, 05.45.Yv, 11.30.-j, 02.30.Oz, 45.20.Jj

\section{Introduction and the system equations}

The semi-discrete integrable nonlinear Schrödinger systems on one-dimensional or quasi-onedimensional lattices $[1-5,23,52,56,57,72]$ play a significant part in modeling a wide variety of phenomena from various branches of physics inasmuch as they might give us a clue what type of nonlinear excitations could be expected when considering real physical situations. It is sufficient to mention that the concept of nonlinear excitations related to one or another model of nonlinear Schrödinger type is applicable to the investigation of nonlinear effects in discrete electric 
transmission lines [36], to the modeling of soliton-mediated energy and charge transport in macromolecules $[9,17,18,47]$ as well as to the theoretical treatment of experimentally observed light patterns in the cross-sections of coupled optical fibers [14,22].

In this respect the semi-discrete integrable nonlinear Schrödinger system with backgroundcontrolled inter-site resonant coupling $[62,66,67]$ is able to find considerable physical applications as a multi-component system with two types of free coupling parameters giving rise to rather unusual properties $[68,69]$ and as the system whose underlying lattice structure is closely related to that of $(1,1)$ boron nanotube [35] belonging to the new class of low-dimensional synthetic materials known as nanoribbons $[25,30,41]$. In present paper we consider the most representative results concerning this system.

Being written in terms of two pairs of basic field variables $q_{+}(n), r_{+}(n)$ and $q_{-}(n), r_{-}(n)$ accompanied by one pair of concomitant field variables $\mu(n), v(n)$, the semi-discrete integrable nonlinear Schrödinger system with background-controlled inter-site resonant coupling (that can be treated also as the integrable nonlinear Schrödinger system given on a ribbon of triangular lattice) reads as follows $[62,66,67]$

$$
\begin{aligned}
& +\mathrm{i} \dot{q}_{+}(n)+\beta q_{-}(n-1)\left[1+q_{+}(n) r_{+}(n)\right]+ \\
& +\alpha q_{+}(n+1)\left[q_{+}(n) r_{-}(n)-v(n)\right]+\alpha\left[q_{-}(n)+q_{+}(n) \mu(n)\right]=0 \\
& \quad-\mathrm{i} \dot{r}_{+}(n)+\alpha r_{-}(n-1)\left[1+r_{+}(n) q_{+}(n)\right]+ \\
& +\beta r_{+}(n+1)\left[r_{+}(n) q_{-}(n)-\mu(n)\right]+\beta\left[r_{-}(n)+r_{+}(n) v(n)\right]=0 \\
& \quad+\mathrm{i} \dot{q}_{-}(n)+\alpha q_{+}(n+1)\left[1+q_{-}(n) r_{-}(n)\right]+ \\
& \quad+\beta q_{-}(n-1)\left[q_{-}(n) r_{+}(n)-\mu(n)\right]+\beta\left[q_{+}(n)+q_{-}(n) v(n)\right]=0 \\
& \quad-\mathrm{i} \dot{r}_{-}(n)+\beta r_{+}(n+1)\left[1+r_{-}(n) q_{-}(n)\right]+ \\
& \quad+\alpha r_{-}(n-1)\left[r_{-}(n) q_{+}(n)-v(n)\right]+\alpha\left[r_{+}(n)+r_{-}(n) \mu(n)\right]=0 \\
& +\mathrm{i} \dot{\mu}(n)+\alpha q_{+}(n+1)\left[r_{+}(n)+r_{-}(n) \mu(n)\right]+ \\
& +\beta\left[q_{+}(n) r_{+}(n)-q_{-}(n) r_{-}(n)\right]-\alpha r_{-}(n-1)\left[q_{-}(n)+q_{+}(n) \mu(n)\right]=0 \\
& -\mathrm{i} \dot{v}(n)+\beta r_{+}(n+1)\left[q_{+}(n)+q_{-}(n) v(n)\right]+ \\
& +\alpha\left[r_{+}(n) q_{+}(n)-r_{-}(n) q_{-}(n)\right]-\beta q_{-}(n-1)\left[r_{-}(n)+r_{+}(n) v(n)\right]=0 .
\end{aligned}
$$

Here the overdot stands for the differentiation with respect to time $\tau$ while the time-dependent parameters $\alpha$ and $\beta$ describe the primary resonant coupling between the field variables on the neighboring sites belonging to the opposite chains of a ladder lattice. The arbitrariness in time dependencies of these parameters is restricted only by the general demand of their complex conjugation $\beta^{*}=\alpha$. The field variables $q_{+}(n)$ and $r_{+}(n)$ (with $r_{+}^{*}(n)=q_{+}(n)$ ) are approximately related to the probability amplitudes to find the $n$-th site on plus-labeled chain of a ladder lattice being excited. Similarly the field variables $q_{-}(n)$ and $r_{-}(n)$ (with $r_{-}^{*}(n)=q_{-}(n)$ ) are approximately related to the probability amplitudes to find the $n$-th site on minus-labeled chain being excited. These two pairs of variables $q_{+}(n), r_{+}(n)$ and $q_{-}(n), r_{-}(n)$ constitute the basic set of dynamic fields given on an 


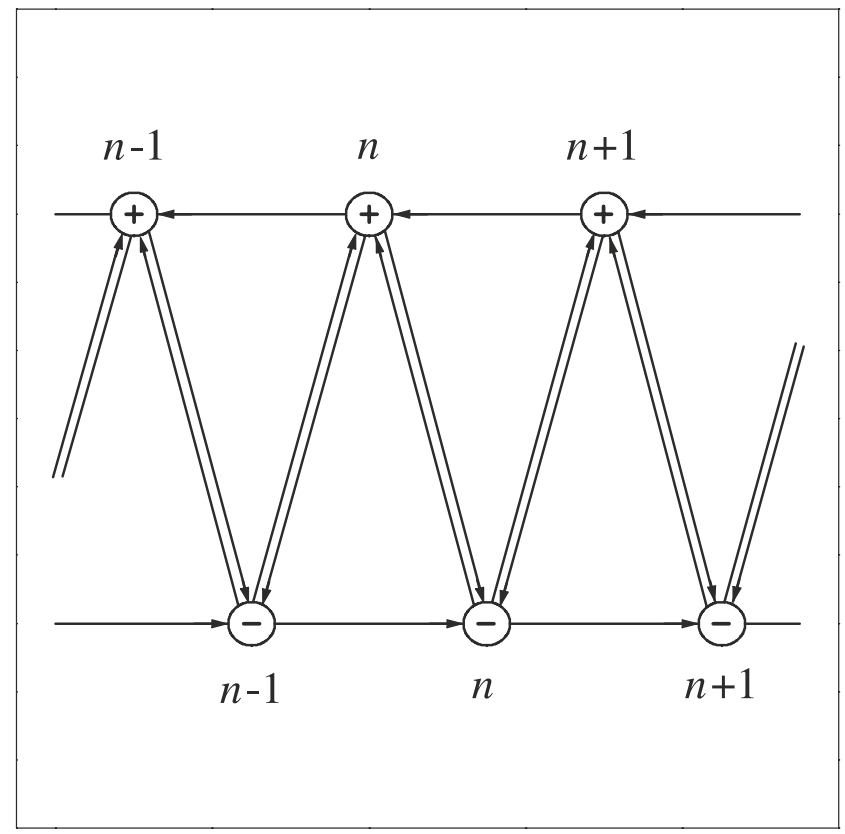

Fig. 1. The fragment of two-leg ladder lattice related to the semi-discrete integrable nonlinear Schrödinger system with background-controlled inter-site resonant coupling (1.1)-(1.6). The circles mark the sites of a ladder lattice. The oblique arrows indicate on the primary inter-site resonant coupling characterized by parameters $\alpha$ or $\beta$. The horizontal arrows indicate on the composite inter-cite resonant coupling characterized by parameters $-\alpha v$ or $-\beta \mu$, where $v=\lim _{|n| \rightarrow \infty} v(n)$ and $\mu=\lim _{|n| \rightarrow \infty} \mu(n)$.

infinite long $(-\infty<n<+\infty)$ ladder lattice with zigzag-like ordered rungs. In so doing the discrete spatial variable $n$ marks the ordinal number of lattice unit cell while the indices + and - serve to distinguish two distinct sites within the same unit cell. By another words the indices + and - can be attributed to two distinct chains (legs) of a ladder lattice. Conversely the variables $\mu(n)$ and $\nu(n)$ (with $v^{*}(n)=\mu(n)$ ) appear as the concomitant set of fields inasmuch they could be excluded from the consideration by means of so-called natural constraints

$$
\begin{aligned}
& \frac{\mu(n)-q_{-}(n) r_{+}(n)}{1+\mu(n) v(n)+q_{+}(n) r_{+}(n)+q_{-}(n) r_{-}(n)}=\frac{\mu}{1+\mu \nu} \\
& \frac{v(n)-q_{+}(n) r_{-}(n)}{1+\mu(n) v(n)+q_{+}(n) r_{+}(n)+q_{-}(n) r_{-}(n)}=\frac{v}{1+\mu \nu}
\end{aligned}
$$

dictated by the very structure of the system equations (1.1)-(1.6) through the system lowest local conservation laws (listed in Section 7). At any rate we must remember that the quantities $\mu(n)$ and $v(n)$ should be considered as functions of basic field variables $q_{+}(n), r_{+}(n)$ and $q_{-}(n), r_{-}(n)$ and do not play an independent part in the system dynamics. As for the resonant coupling between the neighboring sites belonging to the same chain of a ladder lattice it turns out to be nonzero provided the time-independent limiting values $\mu$ and $v$ of concomitant fields $\mu(n)$ and $v(n)$ at both spatial infinities $|n| \rightarrow \infty$ are nonvanishing. As a result the coupling parameters responsible for the longitudinal coupling spring up as essentially composite ones equal to $-\alpha \nu$ and $-\beta \mu$. The term "inter-site resonant coupling" borrowed from the theory of molecular (small-radius) excitations [16] 
assumes a non-dissipative (coherent) coupling responsible for the linear parts of interaction between the excitations of basic fields. The adopted symmetries between the field variables supplemented by the adopted symmetries between the coupling parameters specify the system under consideration (1.1)-(1.6) as the system with the attractive-type nonlinearities.

The spatial arrangement of lattice sites and inter-site resonant bonds related to the system of our interest (1.1)-(1.6) is visualized on Figure 1 as the two-leg ladder lattice that according to modern nanoribbon terminology [21] can be referred to as the simplest triangular-lattice ribbon with linear edges. The quasi-one-dimensionality of such a lattice appears to be a favorable property required in physical applications inasmuch as already the quasi-one-dimensionality (in contrast to the pure one-dimensionality) of real macromolecular lattice structure (in general any lattice structure considered on a spatially microscopic scale) is known to be an indispensable factor for the structure thermodynamic stability [77]. On the other hand namely due to the ladder-like geometry of its underlying lattice structure the system (1.1)-(1.6) (when dealing with the electrically charged excitations) acquires the property to experience an action of external uniform magnetic field in terms of magnetic fluxes threading the elementary (triangular) plackets of lattice ribbon and modeled by the phases of complex-valued coupling parameters treated as the Peierls phases $[44,57,63]$.

\section{System integrability in the Lax sense $[62,66,67]$}

A nonlinear evolutionary system on one-dimensional or quasi-one-dimensional lattice is said to be integrable in the Lax sense [43] provided it permits a semi-discrete zero-curvature representation

$$
\dot{L}(n \mid z)=A(n+1 \mid z) L(n \mid z)-L(n \mid z) A(n \mid z)
$$

in terms of some auxiliary spectral and evolution operators $L(n \mid z)$ and $A(n \mid z)$ with $z$ being the timeindependent complex-valued spectral parameter.

The integrability of semi-discrete integrable nonlinear Schrödinger system with backgroundcontrolled inter-site resonant coupling (1.1)-(1.6) finds its natural confirmation in either of two known choices of auxiliary operators. Originally it had been established by seeking the operators $L(n \mid z)$ and $A(n \mid z)$ as following $4 \times 4$ square matrices [62]

$$
L(n \mid z)=\left(\begin{array}{llll}
z^{2} & g_{11}(n) & f_{12}(n) z & g_{12}(n) z^{-1} \\
g_{11}(n) & z^{2} & g_{12}(n) z^{-1} & f_{12}(n) z \\
g_{21}(n) z^{-1} & f_{21}(n) z & z^{-2} & f_{22}(n) \\
f_{21}(n) z & g_{21}(n) z^{-1} & f_{22}(n) & z^{-2}
\end{array}\right)
$$

and

$$
A(n \mid z)=\left(\begin{array}{llll}
b_{11}(n) z^{2} & c_{11}(n) & b_{12}(n) z & c_{12}(n) z^{-1} \\
c_{11}(n) & b_{11}(n) z^{2} & c_{12}(n) z^{-1} & b_{12}(n) z \\
c_{21}(n) z^{-1} & b_{21}(n) z & c_{22}(n) z^{-2} & b_{22}(n) \\
b_{21}(n) z & c_{21}(n) z^{-1} & b_{22}(n) & c_{22}(n) z^{-2}
\end{array}\right) .
$$

However later the fact of system integrability has been rediscovered relying upon the more simple $2 \times 2$ square matrices $[66,67]$

$$
L(n \mid z)=\left(\begin{array}{ll}
z^{2}+g_{11}(n) & f_{12}(n) z+g_{12}(n) z^{-1} \\
f_{21}(n) z+g_{21}(n) z^{-1} & f_{22}(n)+z^{-2}
\end{array}\right)
$$


and

$$
A(n \mid z)=\left(\begin{array}{ll}
b_{11}(n) z^{2}+c_{11}(n) & b_{12}(n) z+c_{12}(n) z^{-1} \\
b_{21}(n) z+c_{21}(n) z^{-1} & b_{22}(n)+c_{22}(n) z^{-2}
\end{array}\right) .
$$

There is one-to-one correspondence between the two above-mentioned approaches so that irrespective of the adopted choice of auxiliary matrices (2.2) and (2.3) or (2.4) and (2.5) the zero-curvature relation (2.1) yields the general set of integrable nonlinear evolutionary equations [67]

$$
\begin{aligned}
\dot{g}_{11}(n)= & c_{11}(n+1) g_{11}(n)+b_{12}(n+1) g_{21}(n)+c_{12}(n+1) f_{21}(n)- \\
& -g_{11}(n) c_{11}(n)-f_{12}(n) c_{21}(n)-g_{12}(n) b_{21}(n) \\
\dot{f}_{12}(n)= & b_{11}(n+1) g_{12}(n)+c_{11}(n+1) f_{12}(n)+b_{12}(n+1) f_{22}(n)- \\
& -c_{12}(n)-g_{11}(n) b_{12}(n)-f_{12}(n) b_{22}(n) \\
\dot{g}_{12}(n)= & c_{11}(n+1) g_{12}(n)+b_{12}(n+1)+c_{12}(n+1) f_{22}(n)- \\
& -g_{11}(n) c_{12}(n)-f_{12}(n) c_{22}(n)-g_{12}(n) b_{22}(n) \\
\dot{f}_{22}(n)= & b_{21}(n+1) g_{12}(n)+c_{21}(n+1) f_{12}(n)+b_{22}(n+1) f_{22}(n)- \\
- & f_{21}(n) c_{12}(n)-g_{21}(n) b_{12}(n)-f_{22}(n) b_{22}(n) \\
\dot{g}_{21}(n)= & c_{21}(n+1) g_{11}(n)+b_{22}(n+1) g_{21}(n)+c_{22}(n+1) f_{21}(n)- \\
- & g_{21}(n) c_{11}(n)-f_{22}(n) c_{21}(n)-b_{21}(n) \\
\dot{f}_{21}(n) & =b_{21}(n+1) g_{11}(n)+c_{21}(n+1)+b_{22}(n+1) f_{21}(n)- \\
& -f_{21}(n) c_{11}(n)-g_{21}(n) b_{11}(n)-f_{22}(n) b_{21}(n)
\end{aligned}
$$

with the functions $b_{11}(n), c_{11}(n), b_{12}(n), c_{12}(n)$ and $c_{22}(n), b_{22}(n), c_{21}(n), b_{21}(n)$ being specified by the formulas

$$
\begin{gathered}
b_{11}(n)=b_{11} \\
c_{11}(n)=c_{11}-b_{11} f_{12}(n) f_{21}(n-1) \\
b_{12}(n)=b_{11} f_{12}(n) \\
c_{12}(n)=g_{12}(n-1) c_{22} \\
c_{22}(n)=c_{22} \\
b_{22}(n)=b_{22}-c_{22} g_{21}(n) g_{12}(n-1) \\
c_{21}(n)=c_{22} g_{21}(n)
\end{gathered}
$$




$$
b_{21}(n)=f_{21}(n-1) b_{11} .
$$

Here the functions $g_{11}(n), f_{12}(n), g_{12}(n)$ and $f_{22}(n), g_{21}(n), f_{21}(n)$ are understood as the prototype field variables and the parameters $b_{11}, c_{11}$ and $c_{22}, b_{22}$ are permitted to be arbitrary functions of time $\tau$.

The general integrable system (2.6)-(2.19) admits at least two types of complex-valued reductions [67] and two types of real-valued reductions [67]. In particular the reduction [66,67]

$$
\begin{gathered}
g_{11}(n)=\mu(n)=v^{*}(n) \\
f_{12}(n)=+q_{+}(n)=+r_{+}^{*}(n) \\
g_{12}(n)=-q_{-}(n)=-r_{-}^{*}(n) \\
f_{22}(n)=v(n)=\mu^{*}(n) \\
g_{21}(n)=-r_{+}(n)=-q_{+}^{*}(n) \\
f_{21}(n)=+r_{-}(n)=+q_{-}^{*}(n) \\
b_{11}=-\mathrm{i} \alpha=-\mathrm{i} \beta^{*} \\
c_{11}=0 \\
c_{22}=+\mathrm{i} \beta=+\mathrm{i} \alpha^{*} \\
b_{22}=0
\end{gathered}
$$

recovers the multi-component semi-discrete integrable nonlinear Schrödinger system of our main interest (1.1)-(1.6).

It is well known that peculiarities of actual integration of a particular integrable nonlinear system in the framework of inverse scattering transform are dictated by the order of its auxiliary spectral problem. According to Caudrey $[11,12]$ the order of auxiliary spectral problem is determined by the number of distinct eigenvalues $\eta_{j}(z)$ of limiting spectral matrix $L(z)$ (defined either as $L(z)=$ $\lim _{n \rightarrow-\infty} L(n \mid z)$ or as $\left.L(z)=\lim _{n \rightarrow+\infty} L(n \mid z)\right)$, i.e. by the number of distinct solutions of algebraic equation

$$
\operatorname{det}[L(z)-\eta I]=0,
$$

where $I$ is the unity matrix of the same rank as the rank of limiting spectral matrix $L(z)$. Thus the order of auxiliary spectral problem can not exceed the rank of limiting spectral matrix and depends on the very structure of this matrix which is essentially sensitive to the choice of boundary conditions for the field variables. For example, any auxiliary spectral problem linked with one or another of the most demanded multi-component semi-discrete integrable nonlinear Schrödinger 
systems taken at vanishing boundary conditions $[3,23,52,56,57]$ must be treated as the secondorder one despite of being some extended matrix generalization of the basic Ablowitz-Ladik spectral problem $[1,2]$. In contrast the order of auxiliary spectral problem associated with any of multicomponent integrable nonlinear Schrödinger systems taken at non-vanishing boundary conditions $[7,8]$ is usually more higher as compared with its counterpart calculated at vanishing boundary conditions.

In general, when the order of the algebraic equation (2.30) increases the relevant analytical solutions $\eta_{i}(z)$ become cumbersome (for the the cubic equation), impalpable (for the quartic equation) or even impossible (for high order equations). Hence except for some special cases, the soliton theory in practice is forced to deal with low-order auxiliary spectral problems. In this respect it is important to stress that the semi-discrete integrable nonlinear Schrödinger system with background-controlled inter-site resonant coupling (1.1)-(1.6) can be associated not only with the fourth-order auxiliary spectral problem [62] characterized by the four distinct (though explicitly given) eigenvalues

$$
\begin{aligned}
& \eta_{1}(z)=z^{2}+\mu \\
& \eta_{2}(z)=z^{-2}+v \\
& \eta_{3}(z)=z^{2}-\mu \\
& \eta_{4}(z)=z^{-2}-v
\end{aligned}
$$

but also with the second-order auxiliary spectral problem $[66,67]$ characterized only by the two distinct eigenvalues

$$
\begin{gathered}
\eta_{1}(z)=z^{2}+\mu \\
\eta_{2}(z)=z^{-2}+v .
\end{gathered}
$$

\section{Darboux and Bäcklund transformations as the basis for the dressing method of system integration $[66,67]$}

Among the general methods allowing to search for the soliton solutions of an integrable nonlinear system the approach based on the Darboux transformation looks as the most preferable due to its universality and comparative simplicity. The Darboux transformation as applied to the semi-discrete integrable systems has been introduced into soliton theory by Matveev and Salle [38, 39]. Their version of Darboux transformation technique has been successfully applied to the solution of discretized Silin-Tikhonchuk equations [33,34] describing the interaction of Langmuir plasma waves in the case of strong ion-acoustic wave attenuation [48]. Another version of Darboux transformation approach has been suggested by Neugebauer and Meinel [42] and was subsequently applied to the solution of discretized Maxwell-Bloch system [10,45].

The critical analysis of available literature on Darboux transformation method $[6,10,13,19$, $24,33,34,38-40,42,45,74,75]$ has allowed us to accumulate the basic ideas sufficient to generate explicit solutions related to any reduced integrable nonlinear system encoded in suggested general one (2.6)-(2.19). For the sake of generality we present the main steps of relevant Darboux dressing scheme in terms of prototype field variables $g_{11}(n), f_{12}(n), g_{12}(n)$ and $f_{22}(n), g_{21}(n), f_{21}(n)[66,67]$. 
We begin by reminding that the zero-curvature equation (2.1) is obtainable as the compatibility condition between two auxiliary matrix equations

$$
X(n+1 \mid z)=L(n \mid z) X(n \mid z)
$$

and

$$
\dot{X}(n \mid z)=A(n \mid z) X(n \mid z)
$$

referred to as the auxiliary linear problem. Here $X(n \mid z)$ denotes the matrix function whose determinant $\operatorname{det} X(n \mid z)$ can be equal to zero only in a limited number of points on the plane of complex spectral parameter $z$. In what follows we can safely understand the matrices $L(n \mid z)$ and $A(n \mid z)$ as the $2 \times 2$ square matrices invoking (where necessary) their explicit representations (2.4) and (2.5). Thus the matrix $X(n \mid z)$ must be assumed as $2 \times 2$ square matrix too.

Taking into account that the prototype field variables $g_{11}(n), f_{12}(n), g_{12}(n), f_{22}(n), g_{21}(n), f_{21}(n)$ completely determine the spectral $L(n \mid z)$ and evolution $A(n \mid z)$ operators it is possible to distinguish two distinct solutions $g_{11}^{-}(n), f_{12}^{-}(n), g_{12}^{-}(n), f_{22}^{-}(n), g_{21}^{-}(n), f_{21}^{-}(n)$ and $g_{11}^{+}(n), f_{12}^{+}(n), g_{12}^{+}(n), f_{22}^{+}(n)$, $g_{21}^{+}(n), f_{21}^{+}(n)$ of general integrable nonlinear system (2.6)-(2.19) by two sets of matrices $L^{-}(n \mid z)$, $A^{-}(n \mid z)$ and $L^{+}(n \mid z), A^{+}(n \mid z)$ respectively. Then we can formally deal with the minus labeled

$$
\dot{L}^{-}(n \mid z)=A^{-}(n+1 \mid z) L^{-}(n \mid z)-L^{-}(n \mid z) A^{-}(n \mid z)
$$

and plus labeled

$$
\dot{L}^{+}(n \mid z)=A^{+}(n+1 \mid z) L^{+}(n \mid z)-L^{+}(n \mid z) A^{+}(n \mid z)
$$

zero-curvature equations. In view of this agreement there are no principal restrictions to incorporate the minus labeled auxiliary linear problem

$$
\begin{gathered}
X^{-}(n+1 \mid z)=L^{-}(n \mid z) X^{-}(n \mid z) \\
\dot{X}^{-}(n \mid z)=A^{-}(n \mid z) X^{-}(n \mid z)
\end{gathered}
$$

associated with the matrices $L^{-}(n \mid z), A^{-}(n \mid z), X^{-}(n \mid z)$ and plus-labeled auxiliary linear problem

$$
\begin{gathered}
X^{+}(n+1 \mid z)=L^{+}(n \mid z) X^{+}(n \mid z) \\
\dot{X}^{+}(n \mid z)=A^{+}(n \mid z) X^{+}(n \mid z)
\end{gathered}
$$

associated with the matrices $L^{+}(n \mid z), A^{+}(n \mid z), X^{+}(n \mid z)$ into a consolidated Darboux transformation scheme.

By definition [13] the Darboux transformation

$$
X^{+}(n \mid z)=S(n \mid z) X^{-}(n \mid z)
$$

connects the solutions $X^{-}(n \mid z)$ and $X^{+}(n \mid z)$ of minus labeled (3.5), (3.6) and plus labeled (3.7), (3.8) auxiliary linear problems through the Darboux matrix $S(n \mid z)$. The Darboux matrix $S(n \mid z)$ must obey 
to the set of matrix equations

$$
\begin{gathered}
S(n+1 \mid z) L^{-}(n \mid z)=L^{+}(n \mid z) S(n \mid z) \\
\dot{S}(n \mid z)=A^{+}(n \mid z) S(n \mid z)-S(n \mid z) A^{-}(n \mid z)
\end{gathered}
$$

serving as the spatial (3.10) and temporal (3.11) compatibility conditions between the minus labeled (3.5), (3.6) and plus labeled (3.7), (3.8) auxiliary linear problems. It is necessary to stress that these conditions (3.10), (3.11) in combination with minus labeled zero-curvature equation (3.3) lead to the plus labeled one (3.4). Conversely, the temporal compatibility condition (3.11) can be safely treated as the direct consequence of the spatial one (3.10) if both the minus and plus labeled zero-curvature equations (3.3) and (3.4) are regarded as being satisfied.

According to the mnemonic rule quoted by Kuznetsov and Sklyanin [32] the ansatz for the Darboux matrix $S(n \mid z)$ should be chosen in the form similar to that of the spectral matrix $L(n \mid z)$. By another words we have to suppose that the Darboux matrix is given by the expression

$$
S(n \mid z)=\left(\begin{array}{cc}
z^{2}+u_{11}(n) & t_{12}(n) z+u_{12}(n) z^{-1} \\
t_{21}(n) z+u_{21}(n) z^{-1} & t_{22}(n)+z^{-2}
\end{array}\right) .
$$

This ansatz (3.12) prescribes presumably the simplest variant of Darboux matrix relevant to our task and its mission is expected to create one more soliton in a new (plus labeled) solution of nonlinear system as compared with the old (minus labeled) one. When we intend to create two or more solitons the ansatz for the Darboux matrix should be generalized to the form containing the spectral parameter in the same powers as the product of two or more spectral matrices respectively.

Using the adopted ansatz (3.12) for the Darboux matrix the spatial compatibility condition (3.10) can be rewritten as twelve relationships between the functions specifying the Darboux matrix $S(n \mid z)$ and functions involved into the matrices $L^{-}(n \mid z)$ and $L^{+}(n \mid z)$. The respective expressions read as follows

$$
\begin{gathered}
t_{12}(n)=f_{12}^{-}(n) \\
u_{21}(n)=g_{21}^{-}(n) \\
t_{21}(n+1)=f_{21}^{+}(n) \\
u_{12}(n+1)=g_{12}^{+}(n)
\end{gathered}
$$

and

$$
\begin{aligned}
& g_{11}^{-}(n)+t_{12}(n+1) f_{21}^{-}(n)+u_{11}(n+1)=g_{11}^{+}(n)+f_{12}^{+}(n) t_{21}(n)+u_{11}(n) \\
& \begin{aligned}
u_{11}(n+1) g_{11}^{-}(n)+ & u_{12}(n+1) f_{21}^{-}(n)+t_{12}(n+1) g_{21}^{-}(n)= \\
= & g_{11}^{+}(n) u_{11}(n)+f_{12}^{+}(n) u_{21}(n)+g_{12}^{+}(n) t_{21}(n)
\end{aligned} \\
& u_{11}(n+1) f_{12}^{-}(n)+t_{12}(n+1) f_{22}^{-}(n)+g_{12}^{-}(n)= \\
& =g_{11}^{+}(n) t_{12}(n)+f_{12}^{+}(n) t_{22}(n)+u_{12}(n)
\end{aligned}
$$




$$
\begin{aligned}
& u_{11}(n+1) g_{12}^{-}(n)+t_{12}(n+1)+u_{12}(n+1) f_{22}^{-}(n)= \\
& =g_{11}^{+}(n) u_{12}(n)+f_{12}^{+}(n)+g_{12}^{+}(n) t_{22}(n) \\
& f_{22}^{-}(n)+u_{21}(n+1) g_{12}^{-}(n)+t_{22}(n+1)=f_{22}^{+}(n)+g_{21}^{+}(n) u_{12}(n)+t_{22}(n) \\
& t_{22}(n+1) f_{22}^{-}(n)+t_{21}(n+1) g_{12}^{-}(n)+u_{21}(n+1) f_{12}^{-}(n)= \\
& =f_{22}^{+}(n) t_{22}(n)+g_{21}^{+}(n) t_{12}(n)+f_{21}^{+}(n) u_{12}(n) \\
& t_{22}(n+1) g_{21}^{-}(n)+u_{21}(n+1) g_{11}^{-}(n)+f_{21}^{-}(n)= \\
& =f_{22}^{+}(n) u_{21}(n)+g_{21}^{+}(n) u_{11}(n)+t_{21}(n) \\
& t_{22}(n+1) f_{21}^{-}(n)+u_{21}(n+1)+t_{21}(n+1) g_{11}^{-}(n)= \\
& =f_{22}^{+}(n) t_{21}(n)+g_{21}^{+}(n)+f_{21}^{+}(n) u_{11}(n) \text {. }
\end{aligned}
$$

The question arises how to employ these equations (3.13)-(3.24) to generate the new solution of general nonlinear system from the old one.

The most evident but simultaneously unpractical way is to eliminate all mediating Darboux functions $u_{11}(n), t_{12}(n), u_{12}(n), t_{22}(n), u_{21}(n), t_{21}(n)$ and to obtain six equations relating the six functions $g_{11}^{+}(n), f_{12}^{+}(n), g_{12}^{+}(n), f_{22}^{+}(n), g_{21}^{+}(n), f_{21}^{+}(n)$ of new solution with the six functions $g_{11}^{-}(n)$, $f_{12}^{-}(n), g_{12}^{-}(n), f_{22}^{-}(n), g_{21}^{-}(n), f_{21}^{-}(n)$ of old supposedly known one. By definition these six equations establish the Bäcklund transformation between the old and new solutions. Insofar as the resulting formulas for the Bäcklund transformation turn out to be impalpably big we do not present them here. However, the mere fact of their existence instills a firm confidence that the original equations (3.13)-(3.24) must be considered as the innerly noncontradictory tool in developing the Darboux dressing technique.

The standpoint of Darboux dressing method consists in recovering the Darboux functions relying upon certain general spectral properties of adopted Darboux ansatz under the premise that the seed solution (given by the minus labeled field functions) of nonlinear system was preassigned. Should this programm has been accomplished the generation of new solution (given by the plus labeled field functions) is reduced to the solution of purely linear algebraic equations properly chosen among the expanded form (3.13)-(3.24) of spatial compatibility condition (3.10). In the simplest choice (3.12) of Darboux matrix the first two equations (3.13) and (3.14) of the expanded set (3.13)-(3.24) clearly indicates that only four (namely $u_{11}(n), u_{12}(n), t_{22}(n), t_{21}(n)$ ) among six Darboux functions may claim to be initially truly unknown.

To succeed with the Darboux dressing method as applied to our general nonlinear system (2.6)(2.19) it is necessary to analyze the contracted evolution equation

$$
\frac{d}{d \tau} \ln [\operatorname{det} S(n \mid z)]=\operatorname{Sp} A^{+}(n \mid z)-\operatorname{Sp} A^{-}(n \mid z)
$$

deducible from the properly prepared temporal compatibility condition (3.11) by virtue of trace operation. In particular, observing that the combination $\operatorname{Sp} A^{+}(n \mid z)-\operatorname{Sp} A^{-}(n \mid z)$ does not depend on 
the spectral parameter $z$ we can readily conclude that the quantities

$$
\begin{gathered}
W_{+}(n)=t_{22}(n)-t_{21}(n) t_{12}(n) \\
W_{0}(n)=1+t_{22}(n) u_{11}(n)-t_{21}(n) u_{12}(n)-t_{12}(n) u_{21}(n) \\
W_{-}(n)=u_{11}(n)-u_{12}(n) u_{21}(n)
\end{gathered}
$$

entering the expression

$$
\operatorname{det} S(n \mid z)=W_{+}(n) z^{2}+W_{0}(n)+W_{-}(n) z^{-2}
$$

must evolve in a manner leading to the chain of equalities

$$
\frac{d}{d \tau} \ln W_{+}(n)=\frac{d}{d \tau} \ln W_{0}(n)=\frac{d}{d \tau} \ln W_{-}(n) .
$$

As a consequence the functions $W_{+}(n), W_{0}(n), W_{-}(n)$ must differ only by the factors independent of time. In addition the requirement of space uniformity restricts these time independent factors to be independent of space coordinate. Thereafter the formula (3.29) for the determinant of Darboux matrix acquires the form

$$
\operatorname{det} S(n \mid z)=\left(z^{2}+u_{11}\right)\left(z^{-2}+t_{22}\right) \frac{W_{0}(n)}{1+u_{11} t_{22}},
$$

where the parameters $u_{11}$ and $t_{22}$ are set to be time independent, while the functions $t_{12}(n), u_{12}(n)$ and $u_{21}(n), t_{21}(n)$ are assumed to be rapidly vanishing at both spatial infinities. The obtained expression (3.31) shows that all four roots $z_{r}$ of equation $\operatorname{det} S(n \mid z)=0$ are fixed to be independent both of time and coordinate variables.

By virtue of evident identity $\operatorname{det} S\left(n \mid z_{r}\right)=0$ the definition of Darboux transformation (3.9) immediately yields $\operatorname{det} X^{+}\left(n \mid z_{r}\right)=0$. The last equality implies that

$$
\sum_{k=1}^{2} X_{j k}^{+}\left(n \mid z_{r}\right) \varepsilon_{k}\left(z_{r}\right)=0
$$

or the same in more detail

$$
\sum_{i=1}^{2} \sum_{k=1}^{2} S_{j i}\left(n \mid z_{r}\right) X_{i k}^{-}\left(n \mid z_{r}\right) \varepsilon_{k}\left(z_{r}\right)=0,
$$

where $X_{j k}^{+}(n \mid z)$ and $S_{j k}(n \mid z)$ are the matrix elements of matrices $X^{+}(n \mid z)$ and $S(n \mid z)$ respectively. Here all eight spectral parameters $\varepsilon_{k}\left(z_{r}\right) \quad(k=1,2 ; r=1,2,3,4)$ are obliged being independent both of time $\tau$ and coordinate $n$. This statement has a status of rigorously proved theorem.

The obtained formula (3.33) contains the nonuniform set of eight linear algebraic equations $(j=1,2 ; r=1,2,3,4)$ allowing to express the unknown Darboux functions $u_{11}(n), u_{12}(n), t_{22}(n)$, $t_{21}(n)$ via the a priori known constituent parts of Darboux matrix and through the solution $X^{-}(n \mid z)$ of minus labeled auxiliary linear problem (3.5) and (3.6) with the spectral parameters $\varepsilon_{k}\left(z_{r}\right)$ and roots $z_{r}$ taken into account. The solution $X^{-}(n \mid z)$ in turn is completely determined by the minus labeled (seed) solution $g_{11}^{-}(n), f_{12}^{-}(n), g_{12}^{-}(n), f_{22}^{-}(n), g_{21}^{-}(n), f_{21}^{-}(n)$ to the general nonlinear integrable 
system of our interest (2.6)-(2.19). Thus in combination with early obtained expanded formulas (3.13)-(3.24) for the spatial compatibility condition (3.10) we come to the closed Darboux dressing procedure permitting to generate the new solution $g_{11}^{+}(n), f_{12}^{+}(n), g_{12}^{+}(n), f_{22}^{+}(n), g_{21}^{+}(n), f_{21}^{+}(n)$ of general nonlinear system (2.6)-(2.19) from the already known one $g_{11}^{-}(n), f_{12}^{-}(n), g_{12}^{-}(n), f_{22}^{-}(n)$, $g_{21}^{-}(n), f_{21}^{-}(n)$.

In passing it should be mentioned that the spectral coefficients $\varepsilon_{k}\left(z_{r}\right)$ and roots $z_{r}$ arising in Darboux dressing approach play the same part as the discrete spectral data in the method of inverse scattering transform $[2,12,49,51,52,60,61,64]$.

\section{Symmetries of the discrete spectral data $\varepsilon_{k}\left(z_{r}\right)$ and $z_{r}$ related to the nonlinear Schrödinger system with attractive type nonlinearities $[66,67]$}

Considering the multi-component nonlinear Schrödinger system with attractive type nonlinearities (1.1)-(1.6) and taking into account the pairwise complex conjugation of its field variables (2.20)(2.25) as well as its coupling parameters (2.26)-(2.29), it is easy to reveal that the spectral $L(n \mid z)$ and evolution $A(n \mid z)$ matrices (2.4) and (2.5) (with matrix $A(n \mid z)$ (2.5) specified by formulas (2.12)(2.19)) exhibit the following symmetry relations

$$
\begin{gathered}
\sigma_{y}\left[L\left(n \mid 1 / z^{*}\right)\right]^{*} \sigma_{y}=L(n \mid z) \\
\sigma_{z}[L(n \mid-z)] \sigma_{z}=L(n \mid z)
\end{gathered}
$$

and

$$
\begin{gathered}
\sigma_{y}\left[A\left(n \mid 1 / z^{*}\right)\right]^{*} \sigma_{y}=A(n \mid z) \\
\sigma_{z}[A(n \mid-z)] \sigma_{z}=A(n \mid z),
\end{gathered}
$$

where the symbols $\sigma_{y}$ and $\sigma_{z}$ denote the second

$$
\sigma_{y}=\left(\begin{array}{cc}
0 & -\mathrm{i} \\
+\mathrm{i} & 0
\end{array}\right)
$$

and third

$$
\sigma_{z}=\left(\begin{array}{cc}
+1 & 0 \\
0 & -1
\end{array}\right)
$$

Pauli matrices. As a consequence the analogous symmetries

$$
\begin{gathered}
\sigma_{y}\left[X\left(n \mid 1 / z^{*}\right)\right]^{*} \sigma_{y}=X(n \mid z) \\
\sigma_{z}[X(n \mid-z)] \sigma_{z}=X(n \mid z)
\end{gathered}
$$

are valid also for the matrix function $X(n \mid z)$. The similar properties must be true for the plus and minus labeled variants of all three above matrices as well as for the Darboux matrix $S(n \mid z)$ :

$$
\begin{gathered}
\sigma_{y}\left[S\left(n \mid 1 / z^{*}\right)\right]^{*} \sigma_{y}=S(n \mid z) \\
\sigma_{z}[S(n \mid-z)] \sigma_{z}=S(n \mid z) .
\end{gathered}
$$

Below we list several the most representative implications of just established symmetries. 
For instance we have

$$
\begin{aligned}
& t_{21}^{*}(n)=-u_{12}(n) \\
& t_{22}^{*}(n)=+u_{11}(n) \\
& t_{12}^{*}(n)=-u_{21}(n) .
\end{aligned}
$$

These formulas can be shown to support the equality $t_{22}^{*}=u_{11}$ that prompts the following parameterizations

$$
\begin{aligned}
& u_{11}=-\exp (+2 \gamma+2 \mathrm{i} \varkappa) \\
& t_{22}=-\exp (+2 \gamma-2 \mathrm{i} x),
\end{aligned}
$$

where $\gamma$ and $\varkappa$ are real parameters independent of time and coordinate. Then for the roots $z_{r}$ of equation $\operatorname{det} S(n \mid z)=0$ (with $\operatorname{det} S(n \mid z)$ given by the formula (3.31)) we obtain

$$
\begin{array}{r}
z_{1}=\exp (+\gamma+\mathrm{i} \varkappa)=-z_{3} \\
z_{2}=\exp (-\gamma+\mathrm{i} \varkappa)=-z_{4} .
\end{array}
$$

Another important properties

$$
\begin{gathered}
\varepsilon_{1}\left(-z_{r}\right) / \varepsilon_{2}\left(-z_{r}\right)=-\varepsilon_{1}\left(+z_{r}\right) / \varepsilon_{2}\left(+z_{r}\right) \\
\varepsilon_{2}^{*}\left(z_{2}\right) / \varepsilon_{1}^{*}\left(z_{2}\right)=-\varepsilon_{1}\left(z_{1}\right) / \varepsilon_{2}\left(z_{1}\right) \\
\varepsilon_{2}^{*}\left(z_{4}\right) / \varepsilon_{1}^{*}\left(z_{4}\right)=-\varepsilon_{1}\left(z_{3}\right) / \varepsilon_{2}\left(z_{3}\right)
\end{gathered}
$$

ensure the parameterizations

$$
\begin{aligned}
& \varepsilon_{2}\left(z_{2}\right) / \varepsilon_{1}\left(z_{2}\right)=+\exp (+d+\mathrm{i} e)=-\varepsilon_{2}\left(z_{4}\right) / \varepsilon_{1}\left(z_{4}\right) \\
& \varepsilon_{2}\left(z_{1}\right) / \varepsilon_{1}\left(z_{1}\right)=-\exp (-d+\mathrm{i} e)=-\varepsilon_{2}\left(z_{3}\right) / \varepsilon_{1}\left(z_{3}\right)
\end{aligned}
$$

with $d$ and $e$ being real parameters independent of time and coordinate.

The analysis of all available symmetry properties provides a number of simplifications. The most significant among them is the possibility to reduce by half the number of linear algebraic equations encoded in formula (3.33) that contains both known $t_{12}(n), u_{21}(n)$ and unknown $u_{11}(n)$, $u_{12}(n), t_{22}(n), t_{21}(n)$ Darboux functions. Thereafter the number of linear algebraic equations coincides with the number of unknown Darboux functions and the reduced set of equations become readily solvable. 


\section{Multi-component soliton solution for the nonlinear Schrödinger system with attractive type nonlinearities $[66,67]$}

Relying upon the results of previous two sections let us construct the one-soliton solution to the nonlinear Schrödinger system on a triangular-lattice ribbon (1.1)-(1.6) characterized by the attractive type nonlinearities.

For this purpose we adopt the seed (minus labeled) solution as the vacuum one

$$
\begin{aligned}
& f_{12}^{-}(n)=+q_{+}^{-}(n)=0 \\
& g_{12}^{-}(n)=-q_{-}^{-}(n)=0 \\
& g_{11}^{-}(n)=\mu^{-}(n)=\mu \\
& g_{21}^{-}(n)=-r_{+}^{-}(n)=0 \\
& f_{21}^{-}(n)=+r_{-}^{-}(n)=0 \\
& f_{22}^{-}(n)=v^{-}(n)=v .
\end{aligned}
$$

Then the minus labeled spectral and evolution matrices become independent of coordinate variable

$$
\begin{aligned}
& L^{-}(n \mid z)=L^{-}(z) \\
& A^{-}(n \mid z)=A^{-}(z)
\end{aligned}
$$

while the spectral matrix $L^{-}(z)$ is seen to be independent of time variable in addition.

As a consequence the minus labeled zero-curvature equation (3.3) is reduced to the strict commutativity relation

$$
A^{-}(z) L^{-}(z)=L^{-}(z) A^{-}(z)
$$

implying that matrices

$$
L^{-}(z)=\left(\begin{array}{cc}
z^{2}+\mu & 0 \\
0 & v+z^{-2}
\end{array}\right)
$$

and

$$
A^{-}(z)=\left(\begin{array}{cc}
-\mathrm{i} \alpha z^{2} & 0 \\
0 & +\mathrm{i} \beta z^{-2}
\end{array}\right)
$$

share the same set of eigenfunctions

$$
\left|\mathrm{x}_{k}^{-}(z)\right\rangle=\left(\begin{array}{c}
\left\langle 1 \mid \mathrm{x}_{k}^{-}(z)\right\rangle \\
\left\langle 2 \mid \mathrm{x}_{k}^{-}(z)\right\rangle
\end{array}\right)=\left(\begin{array}{c}
\delta_{1 k} \\
\delta_{2 k}
\end{array}\right)
$$

with index $k$ running through the integers 1 and 2 and quantity $\delta_{j k}$ standing for the Kronecker symbol. The eigenvalues $\eta_{k}(z)$ and $\dot{\varphi}_{k}(z)$ of matrices $L^{-}(z)$ and $A^{-}(z)$ are respectively as follows

$$
\eta_{1}(z)=z^{2}+\mu
$$




$$
\eta_{2}(z)=v+z^{-2}
$$

and

$$
\begin{gathered}
\dot{\varphi}_{1}(z)=-\mathrm{i} \alpha z^{2} \\
\dot{\varphi}_{2}(z)=+\mathrm{i} \beta z^{-2} .
\end{gathered}
$$

The former ones $\eta_{1}(z)$ and $\eta_{2}(z)$ are seen to coincide with the eigenvalues (2.35) and (2.36) of limiting spectral operator $L(z)$ defined in Section 2. This fact underlines the generic property of eigenvalues $\eta_{j}(z)$ to be insensitive to the choice of a preferred integration scheme.

Now it can be readily verified that the components $X_{j k}^{-}(n \mid z)$ of solution $X^{-}(n \mid z)$ to the minus labeled auxiliary problem (3.5) and (3.6) should be taken in the form

$$
X_{j k}^{-}(n \mid z)=\left\langle j \mid \mathrm{x}_{k}(z)\right\rangle\left[\eta_{k}(z)\right]^{n} \exp \left[\varphi_{k}(z)\right] \equiv \delta_{j k}\left[\eta_{k}(z)\right]^{n} \exp \left[\varphi_{k}(z)\right]
$$

with the functions $\varphi_{k}(z)$ determined by expressions

$$
\begin{gathered}
\varphi_{1}(z)=-\mathrm{i} A z^{2}+\mathrm{i} \psi \\
\varphi_{2}(z)=+\mathrm{i} B z^{-2}-\mathrm{i} \psi .
\end{gathered}
$$

Here $\psi$ is real constant parameter, while functions $A$ and $B$ are the solutions of first order ordinary differential equations

$$
\dot{A}=\alpha
$$

and

$$
\dot{B}=\beta
$$

restricted by the condition of complex conjugation $B^{*}=A$.

Once the components $X_{j k}^{-}(n \mid z)$ of matrix function $X^{-}(n \mid z)$ are known we have to find the four required Darboux functions $u_{11}(n), u_{12}(n)$ and $t_{22}(n), t_{21}(n)$. This task is realizable by solving two nonuniform sets of reduced equations involved into conditions (3.33) that support the identities $\operatorname{det} X^{+}\left(n \mid z_{r}\right)=0(r=1,2,3,4)$. The results of calculations are as follows

$$
\begin{aligned}
& u_{11}(n)=U_{11}(n) / U_{00}(n) \\
& u_{12}(n)=U_{12}(n) / U_{00}(n)
\end{aligned}
$$

and

$$
\begin{gathered}
t_{22}(n)=T_{22}(n) / T_{00}(n) \\
t_{21}(n)=T_{21}(n) / T_{00}(n),
\end{gathered}
$$

where

$$
\begin{gathered}
U_{11}(n)=X_{11}^{-}\left(n \mid z_{2}\right) X_{22}^{-}\left(n \mid z_{1}\right) \varepsilon_{2}\left(z_{1}\right) \varepsilon_{1}\left(z_{2}\right) z_{2}^{2} / z_{1}-X_{11}^{-}\left(n \mid z_{1}\right) X_{22}^{-}\left(n \mid z_{2}\right) \varepsilon_{2}\left(z_{2}\right) \varepsilon_{1}\left(z_{1}\right) z_{1}^{2} / z_{2} \\
\text { Co-published by Atlantis Press and Taylor \& Francis } \\
\text { Copyright: the authors } \\
264
\end{gathered}
$$


O.O. Vakhnenko / Semi-discrete integrable nonlinear Schrödinger system

$$
\begin{gathered}
U_{12}(n)=X_{11}^{-}\left(n \mid z_{1}\right) X_{11}^{-}\left(n \mid z_{2}\right) \varepsilon_{1}\left(z_{2}\right) \varepsilon_{1}\left(z_{1}\right)\left[z_{1}^{2}-z_{2}^{2}\right] \\
U_{00}(n)=X_{11}^{-}\left(n \mid z_{1}\right) X_{22}^{-}\left(n \mid z_{2}\right) \varepsilon_{2}\left(z_{2}\right) \varepsilon_{1}\left(z_{1}\right) / z_{2}-X_{11}^{-}\left(n \mid z_{2}\right) X_{22}^{-}\left(n \mid z_{1}\right) \varepsilon_{2}\left(z_{1}\right) \varepsilon_{1}\left(z_{2}\right) / z_{1}
\end{gathered}
$$

and

$$
\begin{gathered}
T_{22}(n)=X_{22}^{-}\left(n \mid z_{1}\right) X_{11}^{-}\left(n \mid z_{2}\right) \varepsilon_{1}\left(z_{2}\right) \varepsilon_{2}\left(z_{1}\right) z_{2} / z_{1}^{2}-X_{22}^{-}\left(n \mid z_{2}\right) X_{11}^{-}\left(n \mid z_{1}\right) \varepsilon_{1}\left(z_{1}\right) \varepsilon_{2}\left(z_{2}\right) z_{1} / z_{2}^{2} \\
T_{21}(n)=X_{22}^{-}\left(n \mid z_{2}\right) X_{22}^{-}\left(n \mid z_{1}\right) \varepsilon_{2}\left(z_{1}\right) \varepsilon_{2}\left(z_{2}\right)\left[1 / z_{2}^{2}-1 / z_{1}^{2}\right] \\
T_{00}(n)=X_{22}^{-}\left(n \mid z_{2}\right) X_{11}^{-}\left(n \mid z_{1}\right) \varepsilon_{1}\left(z_{1}\right) \varepsilon_{2}\left(z_{2}\right) z_{1}-X_{22}^{-}\left(n \mid z_{1}\right) X_{11}^{-}\left(n \mid z_{2}\right) \varepsilon_{1}\left(z_{2}\right) \varepsilon_{2}\left(z_{1}\right) z_{2} .
\end{gathered}
$$

Inasmuch as the Darboux functions $u_{11}(n), u_{12}(n)$ and $t_{22}(n), t_{21}(n)$ are already found we have to substitute them into the last ten equations (3.15)-(3.24) derived from the spatial compatibility condition (3.10) and to recover all six functions $q_{+}^{+}(n), q_{-}^{+}(n), \mu^{+}(n)$ and $r_{+}^{+}(n), r_{-}^{+}(n), v^{+}(n)$ involved into one-soliton solution. During the announced calculations we have operated only with six properly chosen equations while the rest four ones have been used for the verification of obtained results. The final formulas for the multi-component one-soliton solution read as follows

$$
\begin{gathered}
q_{+}^{+}(n)=+\sinh (2 \gamma) \frac{\exp \left[+2 \mathrm{i}\left(\varkappa_{+}+\varkappa_{-}\right)(n-\xi-y)+\mathrm{i} \chi\right]}{\cosh \left[2\left(\gamma_{+}+\gamma_{-}\right)(n-s-x)\right]} \\
q_{-}^{+}(n)=-\sinh (2 \gamma) \frac{\exp \left[+2 \mathrm{i}\left(\varkappa_{+}+\varkappa_{-}\right)(n+\xi-y)+\mathrm{i} \chi\right]}{\cosh \left[2\left(\gamma_{+}+\gamma_{-}\right)(n+s-x)\right]} \\
\mu^{+}(n)=\mu-\frac{\exp (+2 \mathrm{i} \varkappa) \sinh (2 \gamma) \sinh \left[2\left(\gamma_{+}+\gamma_{-}-\gamma\right)\right]}{\cosh \left[2\left(\gamma_{+}+\gamma_{-}\right)(n-s-x)\right] \cosh \left[2\left(\gamma_{+}+\gamma_{-}\right)(n+s-x)\right]}
\end{gathered}
$$

and

$$
\begin{gathered}
r_{+}^{+}(n)=+\sinh (2 \gamma) \frac{\exp \left[-2 \mathrm{i}\left(\varkappa_{+}+\varkappa_{-}\right)(n-\xi-y)-\mathrm{i} \chi\right]}{\cosh \left[2\left(\gamma_{+}+\gamma_{-}\right)(n-s-x)\right]} \\
r_{-}^{+}(n)=-\sinh (2 \gamma) \frac{\exp \left[-2 \mathrm{i}\left(\varkappa_{+}+\varkappa_{-}\right)(n+\xi-y)-\mathrm{i} \chi\right]}{\cosh \left[2\left(\gamma_{+}+\gamma_{-}\right)(n+s-x)\right]} \\
v^{+}(n)=v-\frac{\exp (-2 \mathrm{i} x) \sinh (2 \gamma) \sinh \left[2\left(\gamma_{+}+\gamma_{-}-\gamma\right)\right]}{\cosh \left[2\left(\gamma_{+}+\gamma_{-}\right)(n-s-x)\right] \cosh \left[2\left(\gamma_{+}+\gamma_{-}\right)(n+s-x)\right]} .
\end{gathered}
$$

Here the two pairs of real constant parameters $\gamma_{+}, x_{+}$and $\gamma_{-}, x_{-}$are defined by the two sets of equations

$$
\begin{aligned}
& \exp \left(+2 \gamma_{+}+2 \mathrm{i} \varkappa_{+}\right)=\exp (+2 \gamma+2 \mathrm{i} \varkappa)+\mu \\
& \exp \left(+2 \gamma_{+}-2 \mathrm{i} \varkappa_{+}\right)=\exp (+2 \gamma-2 \mathrm{i} \varkappa)+v
\end{aligned}
$$

and

$$
\exp \left(-2 \gamma_{-}+2 \mathrm{i} \varkappa_{-}\right)=\exp (-2 \gamma+2 \mathrm{i} \varkappa)+\mu
$$


O.O. Vakhnenko / Semi-discrete integrable nonlinear Schrödinger system

$$
\exp \left(-2 \gamma_{-}-2 \mathrm{i} \varkappa_{-}\right)=\exp (-2 \gamma-2 \mathrm{i} \varkappa)+v
$$

respectively. Another three real constant parameters $s, \xi, \chi$ are determined by formulas

$$
\begin{gathered}
2 s=1-\frac{\gamma}{\gamma_{+}+\gamma_{-}} \\
2 \xi=1-\frac{\varkappa}{\varkappa_{+}+\varkappa_{-}} \\
\chi=\varkappa_{+}+\varkappa_{-}+4 \varkappa+2 \psi-e .
\end{gathered}
$$

In order to specify the real quantities $x$ and $y$ it was necessary to separate the functions $A$ and $B$ into their real and imaginary parts:

$$
\begin{aligned}
& A=\operatorname{Re}(A)+\mathrm{i} \operatorname{Im}(A) \\
& B=\operatorname{Re}(A)-\mathrm{i} \operatorname{Im}(A) .
\end{aligned}
$$

Having been written at length the functions $x$ and $y$ are given by the expressions

$$
x=-\frac{1}{2}-\frac{\gamma+d / 2}{\gamma_{+}+\gamma_{-}}-\frac{\sinh (2 \gamma)}{\gamma_{+}+\gamma_{-}}[\operatorname{Re}(A) \sin (2 \varkappa)+\operatorname{Im}(A) \cos (2 \varkappa)]
$$

and

$$
y=\frac{\cosh (2 \gamma)}{\varkappa_{+}+\varkappa_{-}}[\operatorname{Re}(A) \cos (2 \varkappa)-\operatorname{Im}(A) \sin (2 \varkappa)]
$$

respectively. Thus the time dependencies of purely real functions $\operatorname{Re}(A)$ and $\operatorname{Im}(A)$ determine the whole soliton dynamics.

In obtained one-soliton solution (5.32)-(5.37) the quantity $\sinh (2 \gamma)$ is seen to characterize the amplitude of each basic soliton component while the parameter $4 \gamma$ manifests itself as the total number of excitations contained in one-soliton solution. The latter fact follows from the formula for the total number of excitations

$$
N=\sum_{m=-\infty}^{\infty} \ln \left[\frac{1+\mu(m) v(m)+q_{+}(m) r_{+}(m)+q_{-}(m) r_{-}(m)}{1+\mu v}\right]
$$

as applied to the one-soliton solution (5.32)-(5.37).

The quantity $x$ is closely related to the mean longitudinal coordinate of soliton wave packet. Assuming the interpretation of quantity $x$ being correct the quantity

$$
v=-\frac{\sinh (2 \gamma)}{\gamma_{+}+\gamma_{-}}[\operatorname{Re}(\alpha) \sin (2 \varkappa)+\operatorname{Im}(\alpha) \cos (2 \varkappa)]
$$

defined by formula $v=\dot{x}$ should be understood as the longitudinal soliton velocity.

The quantity $1 / 2\left|\gamma_{+}+\gamma_{-}\right|$when considered at $\left|\gamma_{+}+\gamma_{-}\right| \ll 1$ estimates the typical width of each basic soliton component in longitudinal direction, though at $\left|\gamma_{+}+\gamma_{-}\right| \gtrsim 1$ such an estimation fails to be correct due to the pronounced manifestation of lattice discreteness through the so-called longitudinal breathing of soliton width caused by soliton longitudinal movement $[59,64]$. The parameter $2\left(x_{+}+x_{-}\right)$is closely related to the soliton momentum. It is also necessary to mention that 
the parameter $2 s$ describes the coordinate splitting between the upper-chain and lower-chain basic soliton components, while the parameter $4\left(\varkappa_{+}+\varkappa_{-}\right) \xi$ determines the phase splitting between those components.

In the case of vanishing background $\mu=0=v$ for the concomitant field variables $\mu(n)$ and $v(n)$ the definitions (5.38)-(5.41) of parameters $\gamma_{+}, x_{+}$and $\gamma_{-}, x_{-}$show that $\gamma_{+}=\gamma=\gamma_{-}$and $\varkappa_{+}=x=x_{-}$. By another words we can understand the parameters $1 / 4 \gamma$ and $4 \varkappa$ as some priming characteristics related to the soliton width and soliton momentum respectively. These priming parameters coincide with the physical ones $1 / 2\left|\gamma_{+}+\gamma_{-}\right|$and $2\left(\varkappa_{+}+\varkappa_{-}\right)$only at $\mu=0=v$.

\section{Recurrent scheme for the successive Darboux transformations [66]}

In order to make the Darboux dressing procedure suitable for successive applications, it is reasonable to treat the superscripts - and + related to the seed and crop solutions as the running ones. In so doing we adopt the following replacements

$$
\begin{aligned}
L^{-}(n \mid z) & \rightarrow L^{(s)}(n \mid z) \\
L^{+}(n \mid z) & \rightarrow L^{(c)}(n \mid z) \\
X^{-}(n \mid z) & \rightarrow X^{(s)}(n \mid z) \\
X^{+}(n \mid z) & \rightarrow X^{(c)}(n \mid z) \\
S(n \mid z) & \rightarrow S^{(c)}(n \mid z) \\
z_{r} & \rightarrow z_{r}(c) \\
\varepsilon_{k}\left(z_{r}\right) & \rightarrow \varepsilon_{k}^{(c)}\left(z_{r}(c)\right)
\end{aligned}
$$

with $c \equiv s+1$ being an arbitrary positive integer. Here the first two records (6.1) and (6.2) assume the respective replacements to be inserted into the involved field amplitudes. As for the $c$-th Darboux matrix $S^{(c)}(n \mid z)$ it must be given by the expression

$$
S^{(c)}(n \mid z)=\left(\begin{array}{cc}
z^{2}+u_{11}^{(c)}(n) & f_{12}^{(s)}(n) z+u_{12}^{(c)}(n) z^{-1} \\
t_{21}^{(c)}(n) z+g_{21}^{(s)}(n) z^{-1} & t_{22}^{(c)}(n)+z^{-2}
\end{array}\right) .
$$

in complete accord with the properties $t_{12}^{(c)}(n)=f_{12}^{(s)}(n)$ and $u_{21}^{(c)}(n)=g_{21}^{(s)}(n)$ that have been established earlier.

Provided the zeroth seed solution $g_{11}^{(0)}(n), f_{12}^{(0)}(n), g_{12}^{(0)}(n), f_{22}^{(0)}(n), g_{21}^{(0)}(n), f_{21}^{(0)}(n)$ and the related zeroth auxiliary matrix-function $X^{(0)}(n \mid z)$ are known, the $N$-th crop solution $g_{11}^{(N)}(n), f_{12}^{(N)}(n), g_{12}^{(N)}(n)$, 
$f_{22}^{(N)}(n), g_{21}^{(N)}(n), f_{21}^{(N)}(n)$ is determined by the following recurrent formulas

$$
\begin{gathered}
g_{11}^{(c)}(n)=G_{11}^{(c)}(n) / D_{00}^{(c)}(n) \\
f_{12}^{(c)}(n)=F_{12}^{(c)}(n) / D_{00}^{(c)}(n) \\
g_{12}^{(c)}(n)=u_{12}^{(c)}(n+1)
\end{gathered}
$$

and

$$
\begin{gathered}
f_{22}^{(c)}(n)=F_{22}^{(c)}(n) / D_{00}^{(c)}(n) \\
g_{21}^{(c)}(n)=G_{21}^{(c)}(n) / D_{00}^{(c)}(n) \\
f_{21}^{(c)}(n)=t_{21}^{(c)}(n+1),
\end{gathered}
$$

where

$$
\begin{aligned}
G_{11}^{(c)}(n) & =\left[u_{11}^{(c)}(n+1) g_{11}^{(s)}(n)+u_{12}^{(c)}(n+1) f_{21}^{(s)}(n)+f_{12}^{(s)}(n+1) g_{21}^{(s)}(n)-u_{12}^{(c)}(n+1) t_{21}^{(c)}(n)\right] t_{22}^{(c)}(n)- \\
& -g_{21}^{(s)}(n)\left[u_{11}^{(c)}(n+1) f_{12}^{(s)}(n)+f_{12}^{(s)}(n+1) f_{22}^{(s)}(n)+g_{12}^{(s)}(n)-u_{12}^{(c)}(n)\right] \\
F_{12}^{(c)}(n) & =\left[u_{11}^{(c)}(n+1) f_{12}^{(s)}(n)+f_{12}^{(s)}(n+1) f_{22}^{(s)}(n)+g_{12}^{(s)}(n)-u_{12}^{(c)}(n)\right] u_{11}^{(c)}(n)- \\
& -f_{12}^{(s)}(n)\left[u_{11}^{(c)}(n+1) g_{11}^{(s)}(n)+u_{12}^{(c)}(n+1) f_{21}^{(s)}(n)+f_{12}^{(s)}(n+1) g_{21}^{(s)}(n)-u_{12}^{(c)}(n+1) t_{21}^{(c)}(n)\right] \\
D_{00}^{(c)}(n)=u_{11}^{(c)}(n) t_{22}^{(c)}(n)-g_{21}^{(s)}(n) f_{12}^{(s)}(n) & \\
F_{22}^{(c)}(n)= & {\left[t_{22}^{(c)}(n+1) f_{22}^{(s)}(n)+t_{21}^{(c)}(n+1) g_{12}^{(s)}(n)+g_{21}^{(s)}(n+1) f_{12}^{(s)}(n)-t_{21}^{(c)}(n+1) u_{12}^{(c)}(n)\right] u_{11}^{(c)}(n)-} \\
& -f_{12}^{(s)}(n)\left[t_{22}^{(c)}(n+1) g_{21}^{(s)}(n)+g_{21}^{(s)}(n+1) g_{11}^{(s)}(n)+f_{21}^{(s)}(n)-t_{21}^{(c)}(n)\right] \\
G_{21}^{(c)}(n) & =\left[t_{22}^{(c)}(n+1) g_{21}^{(s)}(n)+g_{21}^{(s)}(n+1) g_{11}^{(s)}(n)+f_{21}^{(s)}(n)-t_{21}^{(c)}(n)\right] t_{22}^{(c)}(n)- \\
& -g_{21}^{(s)}(n)\left[t_{22}^{(c)}(n+1) f_{22}^{(s)}(n)+t_{21}^{(c)}(n+1) g_{12}^{(s)}(n)+g_{21}^{(s)}(n+1) f_{12}^{(s)}(n)-t_{21}^{(c)}(n+1) u_{12}^{(c)}(n)\right]
\end{aligned}
$$

and the superscript $c$ runs through positive integers from 1 to $N$, while $s=c-1$. Here the $c$-th Darboux functions $u_{11}^{(c)}(n), u_{12}^{(c)}(n)$ and $t_{22}^{(c)}(n), t_{21}^{(c)}(n)$ are given by formulas

$$
\begin{aligned}
& u_{11}^{(c)}(n)=U_{11}^{(c)}(n) / U_{00}^{(c)}(n) \\
& u_{12}^{(c)}(n)=U_{12}^{(c)}(n) / U_{00}^{(c)}(n)
\end{aligned}
$$

and

$$
t_{22}^{(c)}(n)=T_{22}^{(c)}(n) / T_{00}^{(c)}(n)
$$




$$
t_{21}^{(c)}(n)=T_{21}^{(c)}(n) / T_{00}^{(c)}(n)
$$

where

$$
\begin{aligned}
U_{11}^{(c)}(n) & =Y_{1}^{(s)}\left(n \mid z_{2}(c)\right) Y_{2}^{(s)}\left(n \mid z_{1}(c)\right) z_{2}^{2}(c) / z_{1}(c)-Y_{1}^{(s)}\left(n \mid z_{1}(c)\right) Y_{2}^{(s)}\left(n \mid z_{2}(c)\right) z_{1}^{2}(c) / z_{2}(c)+ \\
& +f_{12}^{(s)}(n) Y_{2}^{(s)}\left(n \mid z_{1}(c)\right) Y_{2}^{(s)}\left(n \mid z_{2}(c)\right)\left[z_{2}(c) / z_{1}(c)-z_{1}(c) / z_{2}(c)\right] \\
U_{12}^{(c)}(n) & =Y_{1}^{(s)}\left(n \mid z_{1}(c)\right) Y_{1}^{(s)}\left(n \mid z_{2}(c)\right)\left[z_{1}^{2}(c)-z_{2}^{2}(c)\right]+ \\
& +f_{12}^{(s)}(n)\left[Y_{2}^{(s)}\left(n \mid z_{1}(c)\right) Y_{1}^{(s)}\left(n \mid z_{2}(c)\right) z_{1}(c)-Y_{2}^{(s)}\left(n \mid z_{2}(c)\right) Y_{1}^{(s)}\left(n \mid z_{1}(c)\right) z_{2}(c)\right] \\
U_{00}^{(c)}(n)= & Y_{1}^{(s)}\left(n \mid z_{1}(c)\right) Y_{2}^{(s)}\left(n \mid z_{2}(c)\right) / z_{2}(c)-Y_{1}^{(s)}\left(n \mid z_{2}(c)\right) Y_{2}^{(s)}\left(n \mid z_{1}(c)\right) / z_{1}(c)
\end{aligned}
$$

and

$$
\begin{aligned}
T_{22}^{(c)}(n) & =Y_{2}^{(s)}\left(n \mid z_{1}(c)\right) Y_{1}^{(s)}\left(n \mid z_{2}(c)\right) z_{2}(c) / z_{1}^{2}(c)-Y_{2}^{(s)}\left(n \mid z_{2}(c)\right) Y_{1}^{(s)}\left(n \mid z_{1}(c)\right) z_{1}(c) / z_{2}^{2}(c)+ \\
& +g_{21}^{(s)}(n) Y_{1}^{(s)}\left(n \mid z_{2}(c)\right) Y_{1}^{(s)}\left(n \mid z_{1}(c)\right)\left[z_{2}(c) / z_{1}(c)-z_{1}(c) / z_{2}(c)\right] \\
T_{21}^{(c)}(n) & =Y_{2}^{(s)}\left(n \mid z_{2}(c)\right) Y_{2}^{(s)}\left(n \mid z_{1}(c)\right)\left[1 / z_{2}^{2}(c)-1 / z_{1}^{2}(c)\right]+ \\
& +g_{21}^{(s)}(n)\left[Y_{1}^{(s)}\left(n \mid z_{2}(c)\right) Y_{2}^{(s)}\left(n \mid z_{1}(c)\right) / z_{2}(c)-Y_{1}^{(s)}\left(n \mid z_{1}(c)\right) Y_{2}^{(s)}\left(n \mid z_{2}(c)\right) / z_{1}(c)\right] \\
& T_{00}^{(c)}(n)=Y_{2}^{(s)}\left(n \mid z_{2}(c)\right) Y_{1}^{(s)}\left(n \mid z_{1}(c)\right) z_{1}(c)-Y_{2}^{(s)}\left(n \mid z_{1}(c)\right) Y_{1}^{(s)}\left(n \mid z_{2}(c)\right) z_{2}(c)
\end{aligned}
$$

with the shorthand

$$
Y_{j}^{(s)}\left(n \mid z_{r}(c)\right)=\sum_{k=1}^{2} X_{j k}^{(s)}\left(n \mid z_{r}(c)\right) \varepsilon_{k}^{(c)}\left(z_{r}(c)\right)
$$

being introduced. All these formulas (6.8)-(6.30) should be supplemented with the Darboux transformation relation

$$
X^{(c)}(n \mid z)=S^{(c)}(n \mid z) X^{(s)}(n \mid z)
$$

as an indispensable part of the whole recurrent procedure.

In the case of vacuum zeroth seed solution (5.1)-(5.6) related for the system with attractive type nonlinearities (1.1)-(1.6), when

$$
\begin{gathered}
f_{j k}^{(0)}(n)=v \delta_{j k} \\
g_{j k}^{(0)}(n)=\mu \delta_{j k} \\
X_{j k}^{(0)}(n \mid z)=\delta_{j k}\left[\eta_{k}(z)\right]^{n} \exp \left[\varphi_{k}(z)\right],
\end{gathered}
$$

the general formulas (6.8)-(6.30) yield the first crop solution $g_{11}^{(1)}(n), f_{12}^{(1)}(n), g_{12}^{(1)}(n), f_{22}^{(1)}(n), g_{21}^{(1)}(n)$, $f_{21}^{(1)}(n)$ of one-soliton type (5.32)-(5.37). The question arises whether the $N$-th crop solution based 
on the vacuum zeroth seed solution (6.32), (6.33) can be identified as the $N$-soliton one. Below we provide some convincing arguments in favor of an affirmative answer.

First of all let us parameterize the roots $z_{1}(c)$ and $z_{2}(c)$ of equation $\operatorname{det} S^{(c)}(n \mid z)=0$ by means of the formulas

$$
\begin{gathered}
z_{1}(c)=\exp [+\gamma(c)+\mathrm{i} \varkappa(c)] \\
z_{2}(c)=\exp [-\gamma(c)+\mathrm{i} \gamma(c)],
\end{gathered}
$$

where index $c$ may run through positive integers from 1 to $N$. Taking into account that the quantity $\sinh [2 \gamma(1)]$ determines the amplitude of any basic field function in the case of purely one-soliton solution, we concentrate on the second crop solution characterized by the parameters $\gamma(1)$ and $\gamma(2)$, with parameter $\gamma(1)$ being inherited from the one-soliton seed solution. In order to comprehend the two-soliton structure of the second crop solution, it is sufficient to reveal that in the limiting case of vanishing seed parameter $\gamma(1)$ the solution survives as a newly generated soliton with the amplitude of any basic field function determined by the quantity $\sinh [2 \gamma(2)]$. The simplest way to verify this statement is supported by the one-to-one correspondence between the fundamental set

$$
\sum_{k=1}^{2} S_{j k}^{(1)}\left(n \mid z_{r}(1)\right) X_{k k}^{(0)}\left(n \mid z_{r}(1)\right) \varepsilon_{k}^{(1)}\left(z_{r}(1)\right)=0
$$

associated with the purely one-soliton solution and its counterpart

$$
\sum_{k=1}^{2} S_{j k}^{(2)}\left(n \mid z_{r}(2)\right) X_{k k}^{(0)}\left(n \mid z_{r}(2)\right) \omega_{k}^{(2)}\left(z_{r}(2)\right)=0
$$

associated with the second crop solution taken in the limiting case of vanishing $\gamma(1)$. Precisely, the the structures of Darboux matrices in the former (6.37) and latter (6.38) sets are essentially similar inasmuch as $f_{12}^{(0)}(n)=0=g_{21}^{(0)}(n)$ and $f_{12}^{(1)}(n)=0=g_{21}^{(1)}(n)$. Moreover, the renormalized parameters $\omega^{(2)}\left(z_{r}(2)\right)$ contained in the second set (6.38) are given by the formulas

$$
\begin{gathered}
\omega_{1}^{(2)}\left(z_{r}(2)\right)=\left\{z_{r}^{2}(2)-\exp [+2 \mathrm{i} \varkappa(1)]\right\} \varepsilon_{1}^{(2)}\left(z_{r}(2)\right) \\
\omega_{2}^{(2)}\left(z_{r}(2)\right)=\left\{z_{r}^{-2}(2)-\exp [-2 \mathrm{i} \varkappa(1)]\right\} \varepsilon_{2}^{(2)}\left(z_{r}(2)\right)
\end{gathered}
$$

which are seen to guarantee the symmetry

$$
\left[\omega_{2}^{(2)}\left(z_{2}(2)\right) / \omega_{1}^{(2)}\left(z_{2}(2)\right)\right]^{*}=-\omega_{1}^{(2)}\left(z_{1}(2)\right) / \omega_{2}^{(2)}\left(z_{1}(2)\right)
$$

isomorphic to the original one

$$
\left[\varepsilon_{2}^{(2)}\left(z_{2}(2)\right) / \varepsilon_{1}^{(2)}\left(z_{2}(2)\right)\right]^{*}=-\varepsilon_{1}^{(2)}\left(z_{1}(2)\right) / \varepsilon_{2}^{(2)}\left(z_{1}(2)\right) .
$$

Hence, in view of the symmetry

$$
\left[\varepsilon_{2}^{(1)}\left(z_{2}(1)\right) / \varepsilon_{1}^{(1)}\left(z_{2}(1)\right)\right]^{*}=-\varepsilon_{1}^{(1)}\left(z_{1}(1)\right) / \varepsilon_{2}^{(1)}\left(z_{1}(1)\right),
$$

the properties of parameters $\varepsilon_{k}^{(1)}\left(z_{r}(1)\right)$ and $\omega_{k}^{(2)}\left(z_{r}(2)\right)$ turn out to be similar. At last, the correspondence between the functions $X_{k k}^{(0)}\left(n \mid z_{r}(1)\right)$ and $X_{k k}^{(0)}\left(n \mid z_{r}(2)\right)$ is self-evident.

By induction we can conclude that the $N$-th crop solution based on the vacuum zeroth seed solution should be treated as a $N$-soliton one. 


\section{Infinite set of local densities as a premise for the system integrability in the Liouville sense $[65,67]$}

The Liouville integrability of a nonlinear system given on an infinite spatial support assumes that there exists an infinite set of conserved quantities being in involution in pairs [53]. However already the sole knowledge at least of several local densities provides a good opportunity to study the system dynamics. Here the term "local density" implies the density $\rho(n)$ governed by the local conservation law (discrete-space analogue of continuity equation)

$$
\dot{\rho}(n)=J(n \mid n-1)-J(n+1 \mid n),
$$

where the quantity $J(n+1 / 2 \mid n-1 / 2)$ should be treated as a current. As for the quantity $\sum_{n=-\infty}^{+\infty} \rho(n)$ it is seen to be a conserved one if the values of a current at both spatial infinities coincide. When considering our general integrable system (2.6)-(2.19) we suppose the latter demand being satisfied for the whole infinite set of currents via the proper choice of boundary conditions for the prototype field variables.

In this Section we present explicitly several lowest local conservation laws from the infinite hierarchy related to the general integrable system (2.6)-(2.19). Three of them follow from the universal local conservation law [67]

$$
\frac{d}{d \tau} \ln [\operatorname{det} L(n \mid z)]=\operatorname{Sp} A(n+1 \mid z)-\operatorname{Sp} A(n \mid z)
$$

(being a straightforward consequence of the zero-curvature equation (2.1) under condition that $\operatorname{det} L(n \mid z) \neq 0$ ) while the rest are generated by the direct recursive procedure [67] whose generalized version [65] is suitable for any semi-discrete integrable system linked with the spatially onedimensional auxiliary spectral problem of an arbitrary order.

Here it is worth noticing that the idea to use the direct recursive technique for generating conservation laws had been originated in the pioneering works by Konno, Sanuki and Ichikawa [28] and Wadati, Sanuki and Konno [73] dealing with the continuous integrable nonlinear systems. When having been applied to the semi-discrete integrable nonlinear systems the approach has been reformulated by Tsuchida, Ujino and Wadati [51,52]. However in contrast to the generalized recursive procedure [65] all these articles [28,51,52,73] consider only the simplest versions of direct recursive procedure relying upon the second-order auxiliary spectral problems in the Caudrey sense.

We begin with the local conservation laws emanated from the universal local conservation law (7.2). In so doing we shall use the $2 \times 2$ matrix representations (2.4) and (2.5) for the spectral $L(n \mid z)$ and evolution $A(n \mid z)$ operators with the matrix elements of $A(n \mid z)$ being specified in terms of prototype field variables (2.12)-(2.19). Insofar as the quantity $\operatorname{det} L(n \mid z)$ is a Laurent polynomial in even powers of spectral parameter while the quantity $\operatorname{Sp} A(n+1 \mid z)-\operatorname{Sp} A(n \mid z)$ does not depend of spectral parameter at all the local conservation laws generated by the universal local conservation law (7.2) arise as follows

$$
\begin{gathered}
\dot{\rho}_{+}(n)=J_{0}(n \mid n-1)-J_{0}(n+1 \mid n) \\
\dot{\rho}_{0}(n)=J_{0}(n \mid n-1)-J_{0}(n+1 \mid n) \\
\dot{\rho}_{-}(n)=J_{0}(n \mid n-1)-J_{0}(n+1 \mid n) .
\end{gathered}
$$


Here the local densities $\rho_{+}(n), \rho_{0}(n), \rho_{-}(n)$ are given by expressions

$$
\begin{gathered}
\rho_{+}(n)=\ln \left[f_{22}(n)-f_{21}(n) f_{12}(n)\right] \\
\rho_{0}(n)=\ln \left[1+g_{11}(n) f_{22}(n)-f_{12}(n) g_{21}(n)-g_{12}(n) f_{21}(n)\right] \\
\rho_{-}(n)=\ln \left[g_{11}(n)-g_{12}(n) g_{21}(n)\right]
\end{gathered}
$$

while the currents turn out to be the same for all three local conservation laws since each of them is related to the common quantity

$$
J_{0}(n \mid n-1)=b_{11} f_{12}(n) f_{21}(n-1)+c_{22} g_{21}(n) g_{12}(n-1) .
$$

The structure of lowest local conservation laws (7.3)-(7.5) ensures the chain of equalities $\dot{\rho}_{+}(n)=\dot{\rho}_{0}(n)=\dot{\rho}_{-}(n)$. As a consequence the functions $\exp \left[\rho_{+}(n)\right], \exp \left[\rho_{0}(n)\right], \exp \left[\rho_{-}(n)\right]$ must differ from each other only by the factors independent of time variable $\tau$. To preserve the uniformity of space these factors must be regarded as independent of spatial variable $n$. The statements of this paragraph when applied to the system with the attractive type nonlinearities (1.1)-(1.6) give rise to the early announced natural constrains (1.7) and (1.8) with time-independent background parameters $\mu$ and $v$.

To proceed with the subsequent local conservation laws we briefly summarize the main points of direct recursive technique in its general form [65] dealing with the spectral $L(n \mid z)$ and evolution $A(n \mid z)$ operators given by $R \times R$ square matrices (where $\operatorname{det} L(n \mid z) \neq 0$ ) without any reference on the scattering data of auxiliary spectral problem or on the Hamiltonian structure of an integrable system. Here we do not assume any simplifying restrictions on the order of spectral operator so that it even can coincide with the rank $R$ of respective matrix.

First of all we are obliged to operate with a set of auxiliary functions $\Gamma_{j k}(n \mid z)$ which are proved to comply with the rule

$$
\Gamma_{j i}(n \mid z) \Gamma_{i k}(n \mid z)=\Gamma_{j k}(n \mid z)
$$

and must satisfy to the following set of spatial Riccati equations

$$
\Gamma_{j k}(n+1 \mid z) \sum_{i=1}^{R} L_{k i}(n \mid z) \Gamma_{i k}(n \mid z)=\sum_{i=1}^{R} L_{j i}(n \mid z) \Gamma_{i k}(n \mid z),
$$

where the quantities $L_{j k}(n \mid z)$ denote the matrix elements of spectral operator $L(n \mid z)$. The general property (7.10) imposes constraints onto the auxiliary functions $\Gamma_{j k}(n \mid z)$ so that only $R-1$ of them can be considered as truly independent. For example, dealing with the spectral operator of second order $(R=2)$ it is sufficient to use the single auxiliary function, while relying upon the spectral operator of third order $(R=3)$ we must invoke already two auxiliary functions. 
Another key ingredient of generalized approach is the collection of generating equations

$$
\frac{d}{d \tau} \ln M_{j j}(n \mid z)=B_{j j}(n+1 \mid z)-B_{j j}(n \mid z)
$$

with $M_{j j}(n \mid z)$ and $B_{j j}(n \mid z)$ given by the expressions

$$
M_{j j}(n \mid z)=\sum_{i=1}^{R} L_{j i}(n \mid z) \Gamma_{i j}(n \mid z)
$$

and

$$
B_{j j}(n \mid z)=\sum_{i=1}^{R} A_{j i}(n \mid z) \Gamma_{i j}(n \mid z),
$$

where $A_{j k}(n \mid z)$ are the matrix elements of evolution operator $A(n \mid z)$.

In order to generate the hierarchy of local conservation laws we must seek the solutions to the Riccati equations (7.11) as some appropriate power series with respect to spectral parameter $z$ or inverse spectral parameter $1 / z$. Then inserting the result of calculation into the generating equations (7.12) and collecting the terms with the same powers of $z$ or $1 / z$ we should come to the infinite hierarchy of local conservation laws. The local density in each local conservation law is dictated exclusively by the spectral operator, while the local current by the spectral and evolution operators combined inasmuch as the quantities $\ln M_{j j}(n \mid z)$ should be understood as the generating functions of local densities, while the quantities $B_{j j}(n \mid z)$ should be qualified as the generating functions of local currents.

In the present case of $2 \times 2$ spectral (2.4) and evolution (2.5) matrices $L(n \mid z)$ and $A(n \mid z)$ the set of spatial Riccati equations is reduced to two mutually equivalent equations

$$
\begin{aligned}
& \Gamma_{12}(n+1 \mid z)\left[f_{21}(n) z+g_{21}(n) z^{-1}\right] \Gamma_{12}(n \mid z)+\Gamma_{12}(n+1 \mid z)\left[f_{22}(n)+z^{-2}\right] \\
= & {\left[z^{2}+g_{11}(n)\right] \Gamma_{12}(n \mid z)+f_{12}(n) z+g_{12}(n) z^{-1} }
\end{aligned}
$$

and

$$
\begin{aligned}
& \Gamma_{21}(n+1 \mid z)\left[f_{12}(n) z+g_{12}(n) z^{-1}\right] \Gamma_{21}(n \mid z)+\Gamma_{21}(n+1 \mid z)\left[z^{2}+g_{11}(n)\right] \\
= & {\left[f_{22}(n)+z^{-2}\right] \Gamma_{21}(n \mid z)+f_{21}(n) z+g_{21}(n) z^{-1} . }
\end{aligned}
$$

Thus developing the recurrent procedure at $|z| \rightarrow 0$ it is reasonable to consider the first (7.15) of above two equations and seek the function $\Gamma_{12}(n \mid z)$ in the form

$$
\Gamma_{12}(n \mid z)=z \sum_{k=0}^{\infty} \gamma_{12}(n|0| k) z^{2 k}
$$

Conversely developing the recurrent procedure at $|z| \rightarrow \infty$ it is reasonable to use the second (7.16) of two alternative equations and seek the function $\Gamma_{21}(n \mid z)$ in the form

$$
\Gamma_{21}(n \mid z)=z^{-1} \sum_{k=0}^{\infty} \gamma_{21}(n|\infty| k) z^{-2 k}
$$


The appropriate calculations within each of just announced recurrent schemes yield for the two simplest (but very significant) local conservation laws the following expressions

$$
\begin{aligned}
& \dot{\rho}_{12+}(n)=J_{12+}(n \mid n-1)-J_{12+}(n+1 \mid n) \\
& \dot{\rho}_{21-}(n)=J_{21-}(n \mid n-1)-J_{21-}(n+1 \mid n),
\end{aligned}
$$

where

$$
\begin{aligned}
& \rho_{12+}(n)=f_{22}(n)+g_{21}(n) g_{12}(n-1) \\
& \rho_{21-}(n)=g_{11}(n)+f_{12}(n) f_{21}(n-1)
\end{aligned}
$$

and

$$
\begin{aligned}
J_{12+}(n+1 \mid n) & =c_{22} g_{21}(n+1) g_{12}(n) f_{22}(n) \\
& -c_{22} g_{21}(n+1) f_{12}(n)-f_{21}(n) b_{11} g_{12}(n) \\
& -c_{22} g_{21}(n+1)\left[g_{11}(n)-g_{12}(n) g_{21}(n)\right] g_{12}(n-1) \\
J_{21-}(n+1 \mid n) & =b_{11} f_{12}(n+1) f_{21}(n) g_{11}(n) \\
& -b_{11} f_{12}(n+1) g_{21}(n)-g_{12}(n) c_{22} f_{21}(n) \\
& -b_{11} f_{12}(n+1)\left[f_{22}(n)-f_{21}(n) f_{12}(n)\right] f_{21}(n-1) .
\end{aligned}
$$

Another two admissible but more cumbersome recurrent procedures are based upon the expansion

$$
\Gamma_{21}(n \mid z)=z \sum_{k=0}^{\infty} \gamma_{21}(n|0| k) z^{2 k}
$$

taken at $|z| \rightarrow 0$ and the expansion

$$
\Gamma_{12}(n \mid z)=z^{-1} \sum_{k=0}^{\infty} \gamma_{12}(n|\infty| k) z^{-2 k}
$$

taken at $|z| \rightarrow \infty$ that must be applicable to the second (7.16) and first (7.15) equivalent Riccati equation respectively. The results of calculations reproduce the two local conservation laws (7.3) and (7.5) having already been found in the framework of universal local conservation law (7.2) and yield additionally a series of new ones. The most simple but still significant among them are the 
local conservation laws

$$
\begin{gathered}
\dot{\rho}_{21+}(n)=J_{21+}(n \mid n-1)-J_{21+}(n+1 \mid n) \\
\dot{\rho}_{12-}(n)=J_{12-}(n \mid n-1)-J_{12-}(n+1 \mid n),
\end{gathered}
$$

where

$$
\begin{aligned}
& \rho_{21+}(n)=f_{22}(n)+g_{21}(n+1) g_{12}(n) \\
& \rho_{12-}(n)=g_{11}(n)+f_{12}(n+1) f_{21}(n)
\end{aligned}
$$

and

$$
\begin{aligned}
J_{21+}(n \mid n-1) & =c_{22} f_{22}(n) g_{21}(n) g_{12}(n-1) \\
& -c_{22} f_{21}(n) g_{12}(n-1)-g_{21}(n) b_{11} f_{12}(n) \\
& -c_{22} g_{21}(n+1)\left[g_{11}(n)-g_{12}(n) g_{21}(n)\right] g_{12}(n-1) \\
J_{12-}(n \mid n-1) & =b_{11} g_{11}(n) f_{12}(n) f_{21}(n-1) \\
& -b_{11} g_{12}(n) f_{21}(n-1)-f_{12}(n) c_{22} g_{21}(n) \\
& -b_{11} f_{12}(n+1)\left[f_{22}(n)-f_{21}(n) f_{12}(n)\right] f_{21}(n-1) .
\end{aligned}
$$

Inasmuch as any function of the form $F(n+1 \mid n)-F(n \mid n-1)$ can be always taken as a density in some trivial local conservation law the local densities $\rho_{12+}(n)$ and $\rho_{21+}(n)$ should be considered as physically equivalent. Evidently the same statement concerns the local densities $\rho_{21-}(n)$ and $\rho_{12-}(n)$ too.

\section{Hamiltonian representation of primary nonlinear Schrödinger system with the attractive type nonlinearities $[66,67]$}

Now when the lowest local densities of general semi-discrete integrable nonlinear system are known it is possible to adapt them to the needs of a particular reduced system in order to find out its Hamiltonian representation in terms of properly defined Hamiltonian function and relevant Poisson bracket. In this article we restrict ourselves to the case of nonlinear Schrödinger system on a triangular-lattice ribbon (1.1)-(1.6) characterized by the attractive type nonlinearities.

To be consistent we must remember that the property $\dot{\rho}_{+}(n)=\dot{\rho}_{0}(n)=\dot{\rho}_{-}(n)$ inspires two natural constraints (1.7) and (1.8) implying that the field variables $\mu(n)$ and $v(n)$ (referred to as the concomitant ones) are actually dependent on the basic field variables $q_{+}(n), q_{-}(n)$ and $r_{+}(n), r_{-}(n)$.

As a consequence when starting to apply the general principles of Hamiltonian consideration $[20,37,76]$ we must operate only with the basic equations (i.e. equations (1.1) - (1.4)) and try to rewrite them in the unified form

$$
\dot{\mathrm{y}}_{\lambda}(n)=\sum_{\varkappa=1}^{4} \sum_{m=-\infty}^{\infty} \mathrm{J}_{\lambda \varkappa}(n \mid m) \frac{\partial H}{\partial \mathrm{y}_{\varkappa}(m)}
$$

with the quantity $H$ being the Hamiltonian function and the metric elements $\mathrm{J}_{\lambda \varkappa}(n \mid m)$ subjected to the skew symmetry $\mathrm{J}_{\mathcal{}}(m \mid n)=-\mathrm{J}_{\lambda \varkappa}(n \mid m)$, where $\mathrm{y}_{1}(n), \mathrm{y}_{2}(n), \mathrm{y}_{3}(n), \mathrm{y}_{4}(n)$ is some complete set of 
independent field variables written in the unified form. The above record (8.1) is rather universal inasmuch as it permits a number of particular functional relationships between the unified and basic field variables. In this Section we adopt the simplest linear correspondence between these two sets of field variables given by the relations

$$
\begin{aligned}
& p \mathrm{y}_{1}(n)=q_{-}(n) \\
& p \mathrm{y}_{2}(n)=q_{+}(n) \\
& p \mathrm{y}_{3}(n)=r_{-}(n) \\
& { }_{p} \mathrm{y}_{4}(n)=r_{+}(n) .
\end{aligned}
$$

Here the front-index at quantities $p_{\lambda} \mathrm{y}_{\lambda}$ indicates that actually we deal with the primary basic field variables disguised as the unified ones (i.e. with the basic field variables as they appear in the primary version (1.1)-(1.6) of the semi-discrete integrable nonlinear Schrödinger system with background-controlled inter-site resonant coupling). We shall keep the same front-index at all quantities characterizing the primary system (1.1)-(1.6) in the framework of Hamiltonian treatment.

The experience given by other semi-discrete integrable systems of nonlinear Schrödinger type $[26,31,46,58]$ prompts us to seek the Hamiltonian density of the primary system (1.1)-(1.6) as some superposition of second local densities entering the local conservation laws (see formulas (7.21), (7.22) and (7.29), (7.30) of Section 7 accompanied by the reduction formulas (2.20)-(2.25) of Section 2). This observation gives rise to the following candidate [66, 67]

$$
{ }_{p} H=-\sum_{m=-\infty}^{\infty} \alpha\left[q_{+}(m) r_{-}(m-1)+\mu(m)-\mu\right]-\sum_{m=-\infty}^{\infty} \beta\left[r_{+}(m) q_{-}(m-1)+v(m)-v\right]
$$

on the Hamiltonian function. Then comparing the original basic equations (1.1)-(1.6) with their required unified form (8.1) we obtain the candidates on metric elements ${ }_{p} \mathrm{~J}_{\lambda \varkappa}(n \mid m)$ in terms of basic and concomitant field variables. Their nonzero specimens are written below [67]:

$$
\begin{gathered}
{ }_{p} \mathrm{~J}_{13}(n \mid m)=-\mathrm{i}\left[1+q_{-}(n) r_{-}(n)\right] \delta_{n m} \\
{ }_{p} \mathrm{~J}_{14}(n \mid m)=-\mathrm{i}\left[q_{-}(n) r_{+}(n)-\mu(n)\right] \delta_{n m} \\
{ }_{p} \mathrm{~J}_{23}(n \mid m)=-\mathrm{i}\left[q_{+}(n) r_{-}(n)-v(n)\right] \delta_{n m} \\
\mathrm{~J}_{24}(n \mid m)=-\mathrm{i}\left[1+q_{+}(n) r_{+}(n)\right] \delta_{n m} \\
{ }_{p} \mathrm{~J}_{31}(n \mid m)=+\mathrm{i}\left[1+r_{-}(n) q_{-}(n)\right] \delta_{n m} \\
{ }_{p} \mathrm{~J}_{32}(n \mid m)=+\mathrm{i}\left[r_{-}(n) q_{+}(n)-v(n)\right] \delta_{n m} \\
{ }_{p} \mathrm{~J}_{41}(n \mid m)=+\mathrm{i}\left[r_{+}(n) q_{-}(n)-\mu(n)\right] \delta_{n m}
\end{gathered}
$$


O.O. Vakhnenko / Semi-discrete integrable nonlinear Schrödinger system

$$
{ }_{p} \mathrm{~J}_{42}(n \mid m)=+\mathrm{i}\left[1+r_{+}(n) q_{+}(n)\right] \delta_{n m} .
$$

The rest of metric elements are equal to zero. Once the candidates on metric elements are found we must verify that the quantity $\{F, G\}$ given by formula

$$
\{F, G\}=-\sum_{\lambda=1}^{4} \sum_{\chi=1}^{4} \sum_{n=-\infty}^{\infty} \sum_{m=-\infty}^{\infty} \frac{\partial F}{\partial \mathrm{y}_{\lambda}(n)} \mathrm{J}_{\lambda \varkappa}(n \mid m) \frac{\partial G}{\partial \mathrm{y}_{\varkappa}(m)}
$$

meets all requirements supporting the definition of Poisson bracket $[20,37,76]$. The most critical of them is the demand

$$
\{E,\{F, G\}\}+\{F,\{G, E\}\}+\{G,\{E, F\}\}=0
$$

claiming to be the Jacobi identity. According to the general rule $[20,37,76]$ the above demand $(8.16)$ is tantamount to the set of equations

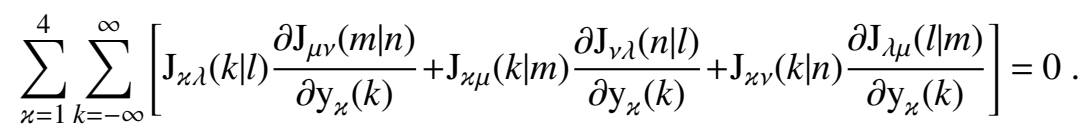

N.B. Here the indices marked by letters $\mu$ and $v$ have nothing to do with the background parameters defined as $\mu=\lim _{|n| \rightarrow \infty} \mu(n)$ and $v=\lim _{|n| \rightarrow \infty} v(n)$.

The direct substitution of early obtained expressions (8.7)-(8.14) for the quantities ${ }_{p} \mathrm{~J}_{\lambda \varkappa}(n \mid m)$ with the use of expressions for derivatives $\partial \mu(n) / \partial_{p} \mathrm{y}_{\varkappa}(n)$ and $\partial v(n) / \partial_{p} \mathrm{y}_{\varkappa}(n)$ following from the natural constraints (1.7) and (1.8) reduces the set of requirements (8.17) into the collection of identities. As a result the Jacobi identity (8.16) turns out to be valid thereby justifying the definition of Poisson bracket (8.15) as well as the choice of Hamiltonian function (8.6).

Using the definition of Poisson bracket (8.15) specified by the formulas for the metric elements (8.7)-(8.14) and taking into account the relationships between the unified and basic field variables (8.2)-(8.5) one can readily calculate all possible Poisson brackets related to the basic $q_{+}(n), r_{+}(n)$, $q_{-}(n), r_{-}(n)$ and concomitant $\mu(n), v(n)$ field variables. Their list is as follows

$$
\begin{gathered}
\left\{q_{+}(m), r_{+}(n)\right\}=+\mathrm{i}\left[1+q_{+}(n) r_{+}(n)\right] \delta_{n m} \\
\left\{q_{+}(m), r_{-}(n)\right\}=+\mathrm{i}\left[q_{+}(n) r_{-}(n)-v(n)\right] \delta_{n m} \\
\left\{q_{-}(m), r_{-}(n)\right\}=+\mathrm{i}\left[1+q_{-}(n) r_{-}(n)\right] \delta_{n m} \\
\left\{q_{-}(m), r_{+}(n)\right\}=+\mathrm{i}\left[q_{-}(n) r_{+}(n)-\mu(n)\right] \delta_{n m} \\
\left\{q_{+}(m), q_{+}(n)\right\}=0=\left\{r_{+}(m), r_{+}(n)\right\} \\
\left\{q_{+}(m), q_{-}(n)\right\}=0=\left\{r_{+}(m), r_{-}(n)\right\} \\
\left\{q_{-}(m), q_{-}(n)\right\}=0=\left\{r_{-}(m), r_{-}(n)\right\} \\
\{\mu(m), v(n)\}=+\mathrm{i}\left[q_{+}(n) r_{+}(n)-q_{-}(n) r_{-}(n)\right] \delta_{n m} \\
\text { Co-published by Atlantis Press and Taylor \& Francis } \\
\text { Copyright: the authors }
\end{gathered}
$$


O.O. Vakhnenko / Semi-discrete integrable nonlinear Schrödinger system

$$
\begin{gathered}
\{\mu(m), \mu(n)\}=0=\{v(m), v(n)\} \\
\left\{\mu(m), r_{-}(n)\right\}=+\mathrm{i}\left[r_{+}(n)+r_{-}(n) \mu(n)\right] \delta_{n m} \\
\left\{\mu(m), q_{+}(n)\right\}=-\mathrm{i}\left[q_{-}(n)+q_{+}(n) \mu(n)\right] \delta_{n m} \\
\left\{v(m), q_{-}(n)\right\}=-\mathrm{i}\left[q_{+}(n)+q_{-}(n) v(n)\right] \delta_{n m} \\
\left\{v(m), r_{+}(n)\right\}=+\mathrm{i}\left[r_{-}(n)+r_{+}(n) v(n)\right] \delta_{n m} \\
\left\{\mu(m), r_{+}(n)\right\}=0=\left\{v(m), q_{+}(n)\right\} \\
\left\{\mu(m), q_{-}(n)\right\}=0=\left\{v(m), r_{-}(n)\right\} .
\end{gathered}
$$

Relying upon these results (8.18)-(8.32) it is not difficult to verify that the nonlinear Schrödinger system on a triangular-lattice ribbon (1.1)-(1.6) permits the concise Hamiltonian representation

$$
\begin{gathered}
\dot{q}_{+}(n)=\left\{{ }_{p} H, q_{+}(n)\right\} \\
\dot{r}_{+}(n)=\left\{{ }_{p} H, r_{+}(n)\right\} \\
\dot{q}_{-}(n)=\left\{{ }_{p} H, q_{-}(n)\right\} \\
\dot{r}_{-}(n)=\left\{{ }_{p} H, r_{-}(n)\right\} \\
\dot{\mu}(n)=\left\{{ }_{p} H, \mu(n)\right\} \\
\dot{v}(n)=\left\{{ }_{p} H, v(n)\right\}
\end{gathered}
$$

with the Hamiltonian function ${ }_{p} H$ given by early adopted formula (8.6).

Though the Hamiltonian function itself (8.6) does not manifests any nonlinear interaction due to be given by the quadratic form with respect to the field variables however the nonlinear interactions still appear in primary dynamic system (1.1)-(1.6) thanks to the highly nonstandard form of relevant Poisson brackets (8.18)-(8.32). The question arises whether it possible to standardize the form of Poisson structure and to carry over all nonlinear interactions directly into the standardized Hamiltonian function. Under some (terminologically veiled but plausible) conditions the positive statement on this problem proclaims the Darboux theorem $[15,20,37,76]$ however it does not give any reasonable prescription how to perform such a standardization. The first rational hint in resolving the puzzle of standardization has been prompted to us by the fact of pronounced criticality of the primary (unstandardized) nonlinear system (1.1)-(1.6) against the governing background parameter $\mu \nu$. We consider the theme of system criticality in the next Section.

It is worth noticing that the nonstandard form of Poisson structure is rather typical property of integrable nonlinear systems associated with the discrete spectral problems. As an example we would like to mention the Ablowitz-Ladik system [1,2] whose nonstandard Poisson structure [26, $31,46]$ hinders the tangible interpretation of its field variables in clear physical terms. 


\section{Criticality of primary dynamic system against the background parameter $[62,68]$}

As the matter of fact the criticality of semi-discrete integrable nonlinear Schrödinger system with background-controlled inter-site resonant coupling (1.1)-(1.6) against the background parameter $\mu \nu$ has been discovered inspecting the spectrum of its low-amplitude excitations [62]. The similar result has been obtained when constructing the analyticity domains of Jost functions of the auxiliary scattering problem associated with the system equations [62].

According to the Caurdey approach to the inverse scattering transform $[12,60,61]$ the lines separating the analyticity domains of distinct Jost functions in the plane of complex-valued spectral parameter $z$ are determined by the collection of equations

$$
\left|\eta_{j}(z)\right|=\left|\eta_{k}(z)\right|,
$$

where $\eta_{j}(z)$ stands for the $j$-th eigenvalue of limiting spectral operator $L(z)$ and indices $j$ and $k$ span all possible combinations in such a way as to prevent their mutual coincidence.

Having been applied to the system of our interest (1.1)-(1.6) and relying upon the $4 \times 4$ matrix representation (2.2) of auxiliary spectral operator $L(n \mid z)$ we come to the set of four distinct eigenvalues (2.31)-(2.34). As a consequence the conditions (9.1) determining the boundaries between the domains of analyticity consist of six equations. The proper analysis of these equations shows the crucial qualitative structure rearrangement of analyticity domains occurring when the background parameter $\mu \nu$ crosses its critical value $\mu \nu=1$ [62]. The Figures 2 and 3 demonstrate the typical subdivisions into the analyticity domains in the plane of phase-adjusted spectral parameter $z \exp (-i \delta)$ respectively at under-critical $\mu \nu<1$ and over-critical $\mu \nu>1$ values of background parameter $\mu \nu$ in the case of fourth order auxiliary spectral operator. Here the real phase parameter $\delta$ is fixed by the relation $\mu / v=\exp (+4 i \delta)$.

The crossover in the arrangement of analyticity domains of the Jost functions is the generic property of the system under study (1.1)-(1.6) and it inevitably takes place also in case of the $2 \times 2$ matrix representation (2.4) of auxiliary spectral operator $L(n \mid z)$ when we have only two distinct eigenvalues (2.35) and (2.36) and hence only one condition (9.1) determining the boundaries between the domains of analyticity. The Figures 4 and 5 demonstrate the typical subdivisions into the analyticity domains in the plane of phase-adjusted spectral parameter $z \exp (-i \delta)$ respectively at under-critical $\mu \nu<1$ and over-critical $\mu \nu>1$ values of background parameter $\mu \nu$ in the case of second order auxiliary spectral operator.

In order to observe the criticality against background parameter $\mu v$ directly in the framework the primary (unstandardized) nonlinear system (1.1)-(1.6) let us rewrite the system two natural constraints (1.7) and (1.8) by means of three formulas

$$
\begin{gathered}
\mu(n)-q_{-}(n) r_{+}(n)=\mu \exp [+\rho(n)] \\
1+\mu(n) v(n)+q_{+}(n) r_{+}(n)+q_{-}(n) r_{-}(n)=(1+\mu v) \exp [+\rho(n)] \\
v(n)-q_{+}(n) r_{-}(n)=v \exp [+\rho(n)],
\end{gathered}
$$

where the common real quantity $\rho(n)$ is supposed to be the total density of excitations on both chains of a ladder lattice. Then combining the above relations (9.2)-(9.4) we come to the expression [68]

$$
\begin{gathered}
2\left[q_{+}(n)+v(n) q_{-}(n)\right]\left[r_{+}(n)+\mu(n) r_{-}(n)\right]+2\left[q_{-}(n)+\mu(n) q_{+}(n)\right]\left[r_{-}(n)+v(n) r_{+}(n)\right]+ \\
+\left[q_{+}(n) r_{+}(n)-q_{-}(n) r_{-}(n)\right]^{2}+[1-\mu(n) v(n)]^{2}=(1-\mu v)^{2} \exp [+2 \rho(n)]
\end{gathered}
$$




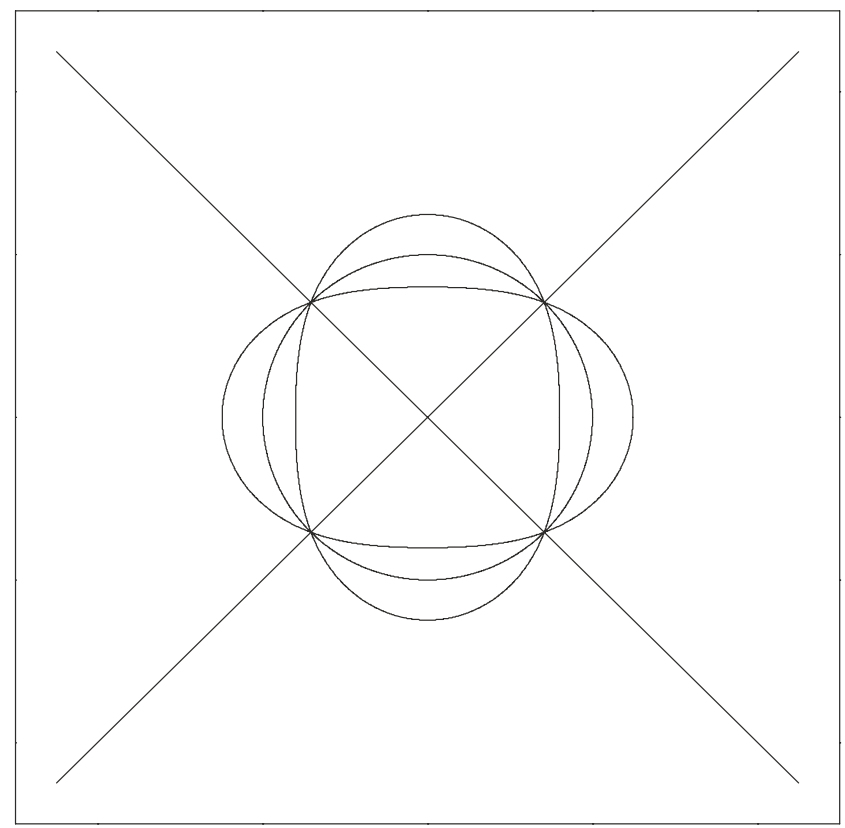

Fig. 2. The typical subdivision into domains of analyticity of Jost functions in the plane of phase-adjusted spectral parameter $z \exp (-i \delta)$ at under-critical values of background parameter $\mu \nu<1$ (s.s. $\mu \nu=\exp (-\pi / 2))$. The case of fourth order auxiliary spectral operator.

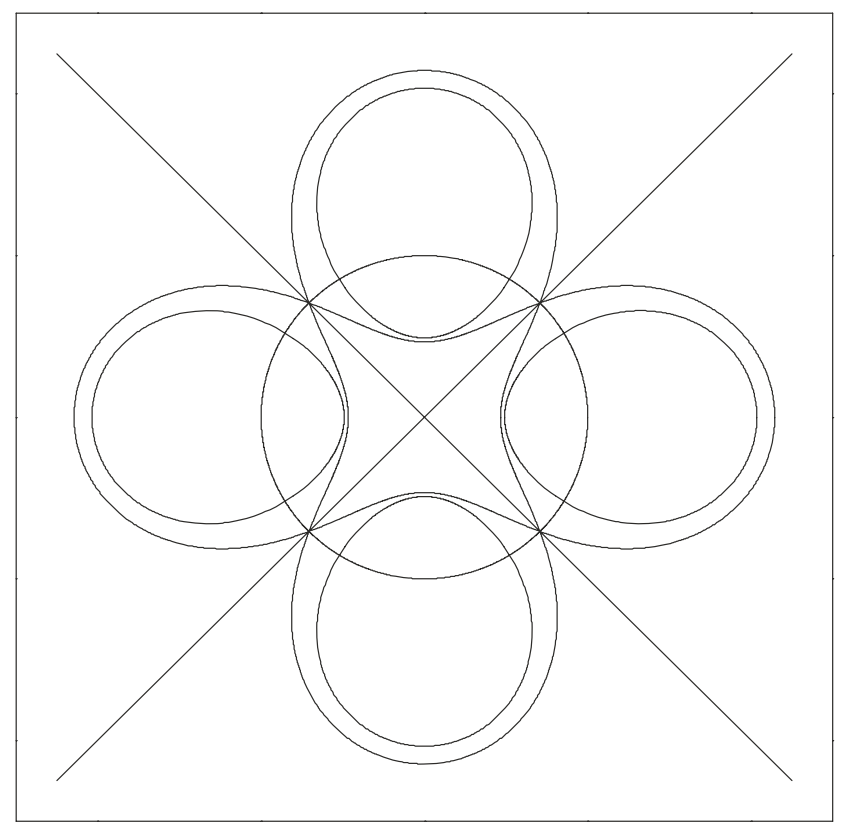

Fig. 3. The typical subdivision into domains of analyticity of Jost functions in the plane of phase-adjusted spectral parameter $z \exp (-i \delta)$ at over-critical values of background parameter $\mu \nu>1$ (s.s. $\mu v=\exp (+\pi / 2)$. The case of fourth order auxiliary spectral operator. 


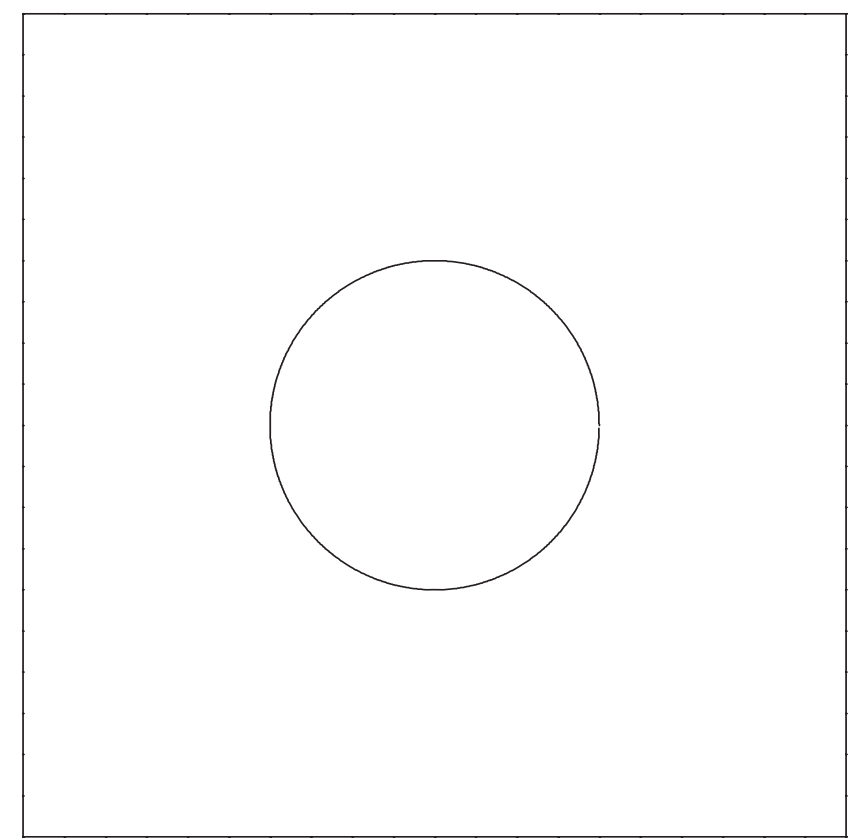

Fig. 4. The subdivision into domains of analyticity of Jost functions in the plane of phase-adjusted spectral parameter $z \exp (-i \delta)$ at under-critical values of background parameter $\mu \nu<1$. The case of second order auxiliary spectral operator.

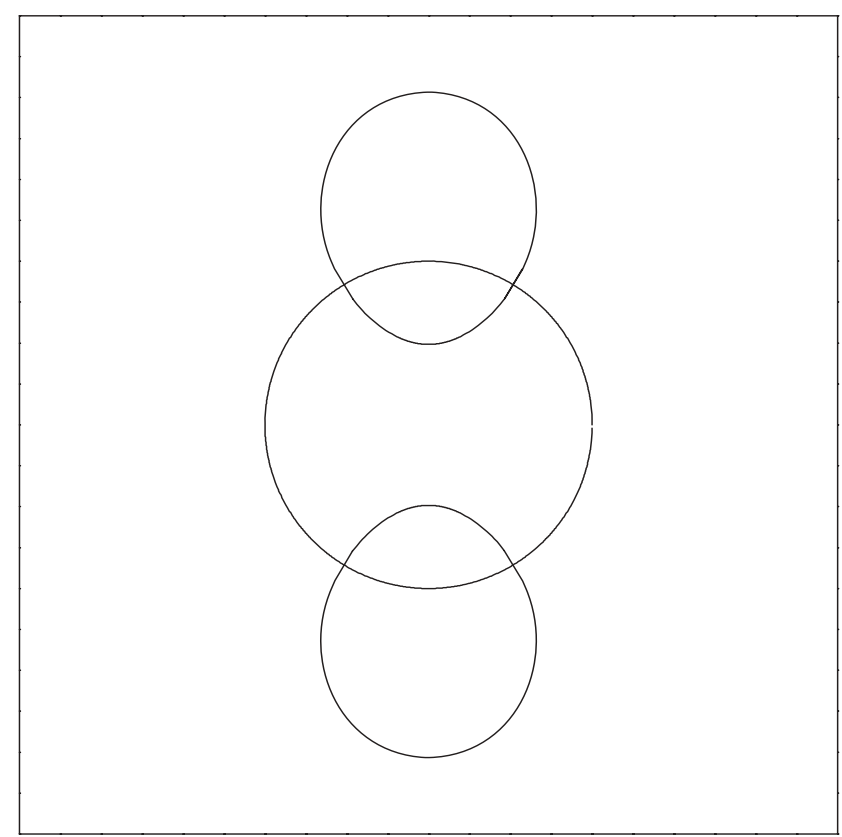

Fig. 5. The typical subdivision into domains of analyticity of Jost functions in the plane of phase-adjusted spectral parameter $z \exp (-i \delta)$ at over-critical values of background parameter $\mu \nu>1($ s.s. $\mu \nu=\exp (+\pi / 2))$. The case of second order auxiliary spectral operator. 
that is seen to be essentially critical against the value of background parameter $\mu \nu$. Precisely at $\mu \nu=$ 1 its right-hand-side part vanishes identically and we are obliged to equalize each term in left-handside part to zero due to the nonnegativity of each such term evident from the inherent symmetries $r_{+}^{*}(n)=q_{+}(n), r_{-}^{*}(n)=q_{-}(n)$ and $v^{*}(n)=\mu(n)$ of primary field amplitudes. These demands valid only at the very critical point $\mu \nu=1$ are tantamount to the extra set of constraints

$$
\begin{gathered}
q_{+}(n)+v(n) q_{-}(n)=0=r_{+}(n)+\mu(n) r_{-}(n) \\
q_{-}(n)+\mu(n) q_{+}(n)=0=r_{-}(n)+v(n) r_{+}(n) \\
\mu(n) v(n)=1
\end{gathered}
$$

that contract the primary multi-component nonlinear dynamic system (1.1)-(1.6) given on a ribbon of triangular lattice with two sites in the unit cell into the two-component nonlinear dynamic system

$$
\begin{aligned}
& +\mathrm{i} \dot{q}(n)+[\alpha q(n+1)+\beta q(n-1)][1+q(n) r(n)]=0 \\
& -\mathrm{i} \dot{r}(n)+[\beta r(n+1)+\alpha r(n-1)][1+r(n) q(n)]=0
\end{aligned}
$$

given on a purely one-dimensional lattice with one site in the unit cell. Here the contracted field variables $q(n)$ and $r(n)$ are defined according to the parametrization formulas

$$
\begin{gathered}
q_{+}(n) \exp [-\mathrm{i}(2 \delta-\pi)(n-1 / 2)]=q(n)=q_{-}(n) \exp [-\mathrm{i}(2 \delta-\pi)(n+1 / 2)] \\
r_{+}(n) \exp [+\mathrm{i}(2 \delta-\pi)(n-1 / 2)]=r(n)=r_{-}(n) \exp [+\mathrm{i}(2 \delta-\pi)(n+1 / 2)] \\
\mu(n) \exp [-2 \mathrm{i} \delta]=1=v(n) \exp [+2 \mathrm{i} \delta]
\end{gathered}
$$

where the real phase parameter $\delta$ is assumed to be time-independent.

Thus in the critical point $\mu \nu=1$ the primary nonlinear integrable system (1.1)-(1.6) shrinks into the more simple system (9.9) and (9.10) that can be referred to as the generalization of integrable Ablowitz-Ladik system [1,2] to the case of time-dependent coupling parameters $\alpha$ and $\beta$ [29,59]. As a result the number of independent field variables is reduced by half while the concomitant field variables are trivialized to the mere constants. However either in under-critical region $\mu \nu<1$ or in over-critical region $\mu v>1$ the system remains being multi-component and it can not be reduced to the more simple (presumably Ablowitz-Ladik) one by any transformation. This statement is in lines with the fact that the structure matrix related to the primary integrable system (1.1)-(1.6) (i.e. structure matrix with the matrix elements ${ }_{p} \mathrm{~J}_{\lambda x}(n \mid m)$ given by formulas (8.7)-(8.14)) degenerates as it will be seen precisely at the critical point $\mu \nu=1$.

Indeed due to the diagonality of primary structure matrix with respect to the spatial indices $n$ and $m$ it is sufficient to deal solely with the determinant ${ }_{p} D(n)$ of local structure matrix, i.e. with the determinant of $4 \times 4$ square matrix whose elements ${ }_{p} \mathrm{~J}_{\lambda \varkappa}(n \mid n)$ are marked by $\lambda$ and $\varkappa$ as the 
only running indices. According to the relations (8.7)-(8.14) specifying the elements ${ }_{p} \mathrm{~J}_{\lambda x}(n \mid m)$ of structure matrix the explicit expression for the local determinant ${ }_{p} D(n)$ is given by formula

$$
{ }_{p} D(n)=\left\{\left[1+q_{+}(n) r_{+}(n)\right]\left[1+q_{-}(n) r_{-}(n)\right]-\left[\mu(n)-q_{-}(n) r_{+}(n)\right]\left[v(n)-q_{+}(n) r_{-}(n)\right]\right\}^{2} .
$$

This expression (9.14) clearly indicates that at critical value $\mu \nu=1$ of background parameter $\mu \nu$ the determinant ${ }_{p} D(n)$ of the local structure matrix and hence the determinant $\prod_{m=-\infty}^{\infty} p(m)$ of the whole structure matrix both turn to zero identically by the virtue of criticality constraints (9.6)-(9.8).

\section{Symmetric sets of intermediate basic field variables and their fundamental Poisson brackets [70]}

Inasmuch as both left-hand-side and right-hand-side parts of criticality-detecting formula (9.5) are essentially nonnegative and moreover the right-hand-side part is a perfect square of purely real function the idea arises to organize the perfect square of some real-valued expression in the lefthand-side part too. This suggestion gives rise to the following substitutions

$$
\begin{aligned}
& q_{+}(n)+v(n) q_{-}(n)=\frac{1-\mu(n) v(n)}{1-\mathrm{u}_{+}(n) \mathrm{v}_{+}(n) \mathrm{u}_{-}(n) \mathrm{v}_{-}(n)}\left[1+\mathrm{u}_{-}(n) \mathrm{v}_{-}(n)\right] \mathrm{u}_{+}(n) \\
& r_{+}(n)+\mu(n) r_{-}(n)=\frac{1-\mu(n) v(n)}{1-\mathrm{u}_{+}(n) \mathrm{v}_{+}(n) \mathrm{u}_{-}(n) \mathrm{v}_{-}(n)}\left[1+\mathrm{u}_{-}(n) \mathrm{v}_{-}(n)\right] \mathrm{v}_{+}(n) \\
& q_{-}(n)+\mu(n) q_{+}(n)=\frac{1-\mu(n) v(n)}{1-\mathrm{u}_{+}(n) \mathrm{v}_{+}(n) \mathrm{u}_{-}(n) \mathrm{v}_{-}(n)}\left[1+\mathrm{u}_{+}(n) \mathrm{v}_{+}(n)\right] \mathrm{u}_{-}(n) \\
& r_{-}(n)+v(n) r_{+}(n)=\frac{1-\mu(n) v(n)}{1-\mathrm{u}_{+}(n) \mathrm{v}_{+}(n) \mathrm{u}_{-}(n) \mathrm{v}_{-}(n)}\left[1+\mathrm{u}_{+}(n) \mathrm{v}_{+}(n)\right] \mathrm{v}_{-}(n)
\end{aligned}
$$

serving to introduce the new basic field variables $\mathrm{u}_{+}(n), \mathrm{v}_{+}(n)$ and $\mathrm{u}_{-}(n), \mathrm{v}_{-}(n)$ instead of the original ones $q_{+}(n), r_{+}(n)$ and $q_{-}(n), r_{-}(n)$. Here the complex conjugation symmetries $r_{+}^{*}(n)=q_{+}(n)$, $r_{-}^{*}(n)=q_{-}(n)$ and $v^{*}(n)=\mu(n)$ of primary field amplitudes ensure the same symmetries $\mathrm{v}_{+}^{*}(n)=$ $\mathrm{u}_{+}(n), \mathrm{v}_{-}^{*}(n)=\mathrm{u}_{-}(n)$ of new basic field amplitudes thereby supporting the nonnegativity of quantities $\mathrm{u}_{+}(n) \mathrm{v}_{+}(n)$ and $\mathrm{u}_{-}(n) \mathrm{v}_{-}(n)$. These new basic field variables $\mathrm{u}_{+}(n), \mathrm{v}_{+}(n)$ and $\mathrm{u}_{-}(n), \mathrm{v}_{-}(n)$ will play an intermediate but very significant part in our forthcoming consideration.

Thus taking the square root of the criticality-detecting formula (9.5) with the use of definitions (10.1)-(10.4) we obtain

$$
\frac{1-\mu(n) v(n)}{1-\mathrm{u}_{+}(n) \mathrm{v}_{+}(n) \mathrm{u}_{-}(n) \mathrm{v}_{-}(n)}\left[1+\mathrm{u}_{+}(n) \mathrm{v}_{+}(n)\right]\left[1+\mathrm{u}_{-}(n) \mathrm{v}_{-}(n)\right]=(1-\mu v) \exp [+\rho(n)] .
$$

Here the signs of square roots have been chosen to ensure the correct limiting values $\mathrm{u}_{+}(n)=q_{+}(n)$, $\mathrm{v}_{+}(n)=r_{+}(n)$ and $\mathrm{u}_{-}(n)=q_{-}(n), \mathrm{v}_{-}(n)=r_{-}(n)$ of intermediate basic field variables $\mathrm{u}_{+}(n), \mathrm{v}_{+}(n)$ and $\mathrm{u}_{-}(n), \mathrm{v}_{-}(n)$ at zero background values $\mu=0=v$ of concomitant fields (see formulas (9.2), (9.4) and (10.1)-(10.4) for the elucidation). Though being the direct consequence of seven previously written formulas (9.2)-(9.4) and (10.1)-(10.4) the above relation (10.5) makes it possible to simplify considerably the calculations of quantities $q_{+}(n), r_{+}(n), q_{-}(n), r_{-}(n), \mu(n), v(n)$ and $\exp [+\rho(n)]$ in 
terms of intermediate field amplitudes $\mathrm{u}_{+}(n), \mathrm{v}_{+}(n)$ and $\mathrm{u}_{-}(n), \mathrm{v}_{-}(n)$. The respective results are as follows

$$
\begin{gathered}
q_{+}(n)=\frac{\mathrm{u}_{+}(n)\left[1+\mu v \mathrm{u}_{-}(n) \mathrm{v}_{-}(n)\right]-v \mathrm{u}_{-}(n)\left[1+\mathrm{u}_{+}(n) \mathrm{v}_{+}(n)\right]}{1-\mu v \mathrm{u}_{+}(n) \mathrm{v}_{+}(n) \mathrm{u}_{-}(n) \mathrm{v}_{-}(n)} \\
r_{+}(n)=\frac{\mathrm{v}_{+}(n)\left[1+\mu v \mathrm{u}_{-}(n) \mathrm{v}_{-}(n)\right]-\mu \mathrm{v}_{-}(n)\left[1+\mathrm{u}_{+}(n) \mathrm{v}_{+}(n)\right]}{1-\mu \nu \mathrm{u}_{+}(n) \mathrm{v}_{+}(n) \mathrm{u}_{-}(n) \mathrm{v}_{-}(n)} \\
q_{-}(n)=\frac{\mathrm{u}_{-}(n)\left[1+\mu v \mathrm{u}_{+}(n) \mathrm{v}_{+}(n)\right]-\mu \mathrm{u}_{+}(n)\left[1+\mathrm{u}_{-}(n) \mathrm{v}_{-}(n)\right]}{1-\mu v \mathrm{u}_{+}(n) \mathrm{v}_{+}(n) \mathrm{u}_{-}(n) \mathrm{v}_{-}(n)} \\
r_{-}(n)=\frac{\mathrm{v}_{-}(n)\left[1+\mu v \mathrm{u}_{+}(n) \mathrm{v}_{+}(n)\right]-v \mathrm{v}_{+}(n)\left[1+\mathrm{u}_{-}(n) \mathrm{v}_{-}(n)\right]}{1-\mu v \mathrm{u}_{+}(n) \mathrm{v}_{+}(n) \mathrm{u}_{-}(n) \mathrm{v}_{-}(n)} \\
\mu(n)=\frac{(1-\mu v) \mathrm{u}_{-}(n) \mathrm{v}_{+}(n)+\mu\left[1-\mathrm{u}_{+}(n) \mathrm{v}_{+}(n) \mathrm{u}_{-}(n) \mathrm{v}_{-}(n)\right]}{1-\mu v \mathrm{u}_{+}(n) \mathrm{v}_{+}(n) \mathrm{u}_{-}(n) \mathrm{v}_{-}(n)} \\
\exp [+\rho(n)]=\frac{\left[1-\mu \mathrm{u}_{+}(n) \mathrm{v}_{-}(n)\right]\left[1-v \mathrm{u}_{-}(n) \mathrm{v}_{+}(n)\right]}{\left[1-\mu v \mathrm{u}_{+}(n) \mathrm{v}_{+}(n) \mathrm{u}_{-}(n) \mathrm{v}_{-}(n)\right]^{2}\left[1+\mathrm{u}_{+}(n) \mathrm{v}_{+}(n)\right]\left[1+\mathrm{u}_{-}(n) \mathrm{v}_{-}(n)\right]} \\
v(n)=\frac{(1-\mu v) \mathrm{u}_{+}(n) \mathrm{v}_{-}(n)+v\left[1-\mathrm{u}_{+}(n) \mathrm{v}_{+}(n) \mathrm{u}_{-}(n) \mathrm{v}_{-}(n)\right]}{1-\mu v \mathrm{u}_{+}(n) \mathrm{v}_{+}(n) \mathrm{u}_{-}(n) \mathrm{v}_{-}(n)}
\end{gathered}
$$

These seven formulas (10.6)-(10.12) have been verified by the direct substitutions into the main determining expressions (9.2)-(9.4) and (10.1)-(10.4) as well as into the auxiliary one (10.5).

The obtained transformation formulas (10.6)-(10.11) allows to exclude the concomitant fields $\mu(n)$ and $v(n)$ from the system dynamics. This positive fact however does not lead automatically to the canonical field variables. First of all at nonzero background values $\mu$ and $v$ of concomitant fields the two sets of intermediate field amplitudes $\mathrm{u}_{+}(n), \mathrm{v}_{+}(n)$ and $\mathrm{u}_{-}(n), \mathrm{v}_{-}(n)$ in the formula (10.12) for $\exp [+\rho(n)]$ are seen to be essentially intermixed. In addition the set of fundamental Poisson brackets related to the intermediate field amplitudes demonstrates essential entanglements between all dynamic variables.

To substantiate the latter statement we turn to the list of Poisson brackets (8.18)-(8.32) related to the primary field variables $q_{+}(n), r_{+}(n), q_{-}(n), r_{-}(n)$ and $\mu(n), v(n)$. As it has been sown in Section 9 the structure matrix responsible for these brackets (8.18)-(8.32) is degenerate only at the critical value $\mu \nu=1$ of background parameter $\mu \nu$. Thus outside the critical point (i.e. at $\mu \nu \neq 1$ ) we can safely apply all necessary formulas from the above list (8.18)-(8.32) when calculating the fundamental Poisson brackets related to the intermediate field variables $\mathrm{u}_{+}(n), \mathrm{v}_{+}(n), \mathrm{u}_{-}(n), \mathrm{v}_{-}(n)$. 
The established results are summarized by the relations

$$
\begin{gathered}
\left\{\mathrm{u}_{+}(m), \mathrm{v}_{+}(n)\right\}=\frac{\mathrm{i}}{1-\mu \nu}\left[1+\mathrm{u}_{+}(n) \mathrm{v}_{+}(n)\right]\left[1+\mu \nu \mathrm{u}_{+}(n) \mathrm{v}_{+}(n)\right] \delta_{n m} \\
\left\{\mathrm{u}_{+}(m), \mathrm{v}_{-}(n)\right\}=\frac{\mathrm{i} v}{1-\mu \nu} \frac{1-\mu \mathrm{u}_{+}(n) \mathrm{v}_{-}(n)}{1-v \mathrm{u}_{-}(n) \mathrm{v}_{+}(n)}\left[1+\mathrm{u}_{+}(n) \mathrm{v}_{+}(n)\right]\left[1+\mathrm{u}_{-}(n) \mathrm{v}_{-}(n)\right] \delta_{n m} \\
\left\{\mathrm{u}_{-}(m), \mathrm{v}_{-}(n)\right\}=\frac{\mathrm{i}}{1-\mu \nu}\left[1+\mathrm{u}_{-}(n) \mathrm{v}_{-}(n)\right]\left[1+\mu v \mathrm{u}_{-}(n) \mathrm{v}_{-}(n)\right] \delta_{n m} \\
\left\{\mathrm{u}_{-}(m), \mathrm{v}_{+}(n)\right\}=\frac{\mathrm{i} \mu}{1-\mu \nu} \frac{1-v \mathrm{u}_{-}(n) \mathrm{v}_{+}(n)}{1-\mu \mathrm{u}_{+}(n) \mathrm{v}_{-}(n)}\left[1+\mathrm{u}_{+}(n) \mathrm{v}_{+}(n)\right]\left[1+\mathrm{u}_{-}(n) \mathrm{v}_{-}(n)\right] \delta_{n m} \\
\left\{\mathrm{u}_{+}(m), \mathrm{u}_{+}(n)\right\}=\left\{\mathrm{u}_{+}(m), \mathrm{u}_{-}(n)\right\}=\left\{\mathrm{u}_{-}(m), \mathrm{u}_{-}(n)\right\}=0 \\
\left\{\mathrm{v}_{+}(m), \mathrm{v}_{+}(n)\right\}=\left\{\mathrm{v}_{+}(m), \mathrm{v}_{-}(n)\right\}=\left\{\mathrm{v}_{-}(m), \mathrm{v}_{-}(n)\right\}=0 .
\end{gathered}
$$

In the course of rather long and tedious calculations of these formulas (10.13)-(10.18) we were obligated to find the expressions for the intermediate field variables $\mathrm{u}_{+}(n), \mathrm{v}_{+}(n), \mathrm{u}_{-}(n), \mathrm{v}_{-}(n)$ in terms of the primary ones $q_{+}(n), r_{+}(n), q_{-}(n), r_{-}(n), \mu(n), v(n)$ using the original definitions (10.1)(10.4) and then to proceed step by step through the whole list of required fundamental Poisson brackets taking into account the expressions (10.6)-(10.11) for the old field variables in terms of the new ones.

The nonzero elements of structure matrix related to the intermediate field variables are given by formulas

$$
\begin{aligned}
& { }_{i} \mathbf{J}_{13}(n \mid m)=-{ }_{i} \mathbf{J}_{31}(m \mid n)=-\left\{\mathbf{u}_{-}(m), \mathbf{v}_{-}(n)\right\} \\
& { }_{i} \mathbf{J}_{14}(n \mid m)=-{ }_{i} \mathbf{J}_{41}(m \mid n)=-\left\{\mathbf{u}_{-}(m), \mathbf{v}_{+}(n)\right\} \\
& { }_{i} \mathbf{J}_{23}(n \mid m)=-{ }_{i} \mathbf{J}_{32}(m \mid n)=-\left\{\mathbf{u}_{+}(m), \mathbf{v}_{-}(n)\right\} \\
& { }_{i} \mathbf{J}_{24}(n \mid m)=-{ }_{i} \mathbf{J}_{42}(m \mid n)=-\left\{\mathbf{u}_{+}(m), \mathbf{v}_{+}(n)\right\} .
\end{aligned}
$$

The respective local structure determinant

$$
{ }_{i} D(n)=\left\{\left\{\mathrm{u}_{+}(n), \mathrm{v}_{+}(n)\right\}\left\{\mathrm{u}_{-}(n), \mathrm{v}_{-}(n)\right\}-\left\{\mathrm{u}_{+}(n), \mathrm{v}_{-}(n)\right\}\left\{\mathrm{u}_{-}(n), \mathrm{v}_{+}(n)\right\}\right\}^{2}
$$

acquires the form

$$
{ }_{i} D(n)=\frac{\left[1+\mathrm{u}_{+}(n) \mathrm{v}_{+}(n)\right]^{2}\left[1+\mathrm{u}_{-}(n) \mathrm{v}_{-}(n)\right]^{2}}{(1-\mu v)^{2}\left[1-\mu \nu \mathrm{u}_{+}(n) \mathrm{v}_{+}(n) \mathrm{u}_{-}(n) \mathrm{v}_{-}(n)\right]^{2}}
$$

that is seen to be divergent when the background parameter $\mu \nu$ tends to unity.

Recollecting that the local structure determinant ${ }_{p} D(n)(9.14)$ related to the primary field variables tends to zero when the background parameter $\mu \nu$ tends to unity it is reasonable to seek some 
compromise between the intermediate and primary field variables and to introduce one or another asymmetric set of field variables via an appropriate choice of two complementary subsets. The idea of asymmetry is supported also by the already proved fact that the number of independent field variables is reduced by half when the background parameter $\mu v$ takes its critical value equal to unity. Thus we may expect that the subsystem described by the intermediate field amplitudes should become unexcited in the critical point. In the next Section we shall start to develop the idea of symmetry breaking by introducing the two possible variants of primary-intermediate field variables explicitly.

\section{Two variants of primary-intermediate field variables [70]}

The symmetry between the two subsets of intermediate field variables $\mathrm{u}_{+}(n), \mathrm{v}_{+}(n)$ and $\mathrm{u}_{-}(n), \mathrm{v}_{-}(n)$ assumes that replacing one or another subset by its primary counterpart we can operate with either of two sets $q_{+}(n), r_{+}(n), \mathrm{u}_{-}(n), \mathrm{v}_{-}(n)$ or $q_{-}(n), r_{-}(n), \mathrm{u}_{+}(n), \mathrm{v}_{+}(n)$ of blended primary-intermediate field variables separately on an equal footing.

Let us begin with the first variant of primary-intermediate field variables $q_{+}(n), r_{+}(n), \mathrm{u}_{-}(n)$, $\mathrm{v}_{-}(n)$. In order to reformulate the system dynamics in terms of these variables we must obtain the respective representations for the minus-labeled basic fields $q_{-}(n), r_{-}(n)$ and the concomitant fields $\mu(n), v(n)$ as well as for the quantity $\exp [+\rho(n)]$. In so doing it is helpful to use the following relationships

$$
\begin{aligned}
& \frac{q_{-}(n)+\mu(n) q_{+}(n)}{1+q_{+}(n) r_{+}(n)}=\frac{(1-\mu v) \mathrm{u}_{-}(n)}{1+\mu v \mathrm{u}_{-}(n) \mathrm{v}_{-}(n)} \\
& \frac{r_{-}(n)+v(n) r_{+}(n)}{1+q_{+}(n) r_{+}(n)}=\frac{(1-\mu v) \mathrm{v}_{-}(n)}{1+\mu v \mathrm{u}_{-}(n) \mathrm{v}_{-}(n)}
\end{aligned}
$$

compatible with the transformation formulas (10.1)-(10.4) and (10.6)-(10.11) considered in Section 10. The appropriate calculations yield

$$
\begin{gathered}
q_{-}(n)=\frac{(1-\mu v) \mathrm{u}_{-}(n)}{1+\mu \nu \mathrm{u}_{-}(n) \mathrm{v}_{-}(n)}-\mu q_{+}(n) \frac{1+\mathrm{u}_{-}(n) \mathrm{v}_{-}(n)}{1+\mu \nu \mathrm{u}_{-}(n) \mathrm{v}_{-}(n)} \\
r_{-}(n)=\frac{(1-\mu v) \mathrm{v}_{-}(n)}{1+\mu v \mathrm{u}_{-}(n) \mathrm{v}_{-}(n)}-v r_{+}(n) \frac{1+\mathrm{u}_{-}(n) \mathrm{v}_{-}(n)}{1+\mu v \mathrm{u}_{-}(n) \mathrm{v}_{-}(n)} \\
\mu(n)=\frac{(1-\mu v) \mathrm{u}_{-}(n) r_{+}(n)}{1+\mu v \mathrm{u}_{-}(n) \mathrm{v}_{-}(n)}+\mu \frac{1+\mathrm{u}_{-}(n) \mathrm{v}_{-}(n)}{1+\mu v \mathrm{u}_{-}(n) \mathrm{v}_{-}(n)} \\
v(n)=\frac{(1-\mu v) q_{+}(n) \mathrm{v}_{-}(n)}{1+\mu v \mathrm{u}_{-}(n) \mathrm{v}_{-}(n)}+v \frac{1+\mathrm{u}_{-}(n) \mathrm{v}_{-}(n)}{1+\mu v \mathrm{u}_{-}(n) \mathrm{v}_{-}(n)} \\
\exp [+\rho(n)]=\left[1+q_{+}(n) r_{+}(n)\right] \frac{1+\mathrm{u}_{-}(n) \mathrm{v}_{-}(n)}{1+\mu v \mathrm{u}_{-}(n) \mathrm{v}_{-}(n)}
\end{gathered}
$$

While preferring the second variant of primary-intermediate field variables $q_{-}(n), r_{-}(n), \mathrm{u}_{+}(n)$, $\mathrm{v}_{+}(n)$ we must obtain the respective representations for the plus-labeled basic fields $q_{+}(n), r_{+}(n)$ and 
the concomitant fields $\mu(n), v(n)$ as well as for the quantity $\exp [+\rho(n)]$. In so doing it is helpful to use the following relationships

$$
\begin{aligned}
& \frac{q_{+}(n)+v(n) q_{-}(n)}{1+q_{-}(n) r_{-}(n)}=\frac{(1-\mu v) \mathrm{u}_{+}(n)}{1+\mu v \mathrm{u}_{+}(n) \mathrm{v}_{+}(n)} \\
& \frac{r_{+}(n)+\mu(n) r_{-}(n)}{1+q_{-}(n) r_{-}(n)}=\frac{(1-\mu v) \mathrm{v}_{+}(n)}{1+\mu v \mathrm{u}_{+}(n) \mathrm{v}_{+}(n)}
\end{aligned}
$$

compatible with the transformation formulas (10.1)-(10.4) and (10.6)-(10.11) considered in Section 10. The appropriate calculations yield

$$
\begin{gathered}
q_{+}(n)=\frac{(1-\mu v) \mathrm{u}_{+}(n)}{1+\mu \nu \mathrm{u}_{+}(n) \mathrm{v}_{+}(n)}-v q_{-}(n) \frac{1+\mathrm{u}_{+}(n) \mathrm{v}_{+}(n)}{1+\mu v \mathrm{u}_{+}(n) \mathrm{v}_{+}(n)} \\
r_{+}(n)=\frac{(1-\mu v) \mathrm{v}_{+}(n)}{1+\mu v \mathrm{u}_{+}(n) \mathrm{v}_{+}(n)}-\mu r_{-}(n) \frac{1+\mathrm{u}_{+}(n) \mathrm{v}_{+}(n)}{1+\mu v \mathrm{u}_{+}(n) \mathrm{v}_{+}(n)} \\
\mu(n)=\frac{(1-\mu v) q_{-}(n) \mathrm{v}_{+}(n)}{1+\mu v \mathrm{u}_{+}(n) \mathrm{v}_{+}(n)}+\mu \frac{1+\mathrm{u}_{+}(n) \mathrm{v}_{+}(n)}{1+\mu v \mathrm{u}_{+}(n) \mathrm{v}_{+}(n)} \\
v(n)=\frac{(1-\mu v) \mathrm{u}_{+}(n) r_{-}(n)}{1+\mu v \mathrm{u}_{+}(n) \mathrm{v}_{+}(n)}+v \frac{1+\mathrm{u}_{+}(n) \mathrm{v}_{+}(n)}{1+\mu v \mathrm{u}_{+}(n) \mathrm{v}_{+}(n)} \\
\exp [+\rho(n)]=\left[1+q_{-}(n) r_{-}(n)\right] \frac{1+\mathrm{u}_{+}(n) \mathrm{v}_{+}(n)}{1+\mu \nu \mathrm{u}_{+}(n) \mathrm{v}_{+}(n)}
\end{gathered}
$$

Looking at formulas (11.3)-(11.7) and (11.10)-(11.14) establishing respectively the first and the second variant of primary-intermediate field variables $q_{+}(n), r_{+}(n), \mathrm{u}_{-}(n), \mathrm{v}_{-}(n)$ and $q_{-}(n)$, $r_{-}(n), \mathrm{u}_{+}(n), \mathrm{v}_{+}(n)$ we clearly see that the alternative expressions (11.7) and (11.14) for the quantity $\exp [+\rho(n)]$ both are factorized. This observation gives us the strong recommendation how to subdivide the whole dynamic system into two rigorously determined subsystems. Moreover each of formulas (11.7) and (11.14) for the quantity $\exp [+\rho(n)]$ strictly detects the character of excitations in both subsystems. Thus at the under-critical values $\mu \nu<1$ of background parameter $\mu v$ both subsystems are of bright-excitation type and the quantity $\rho(n)$ should be treated as the total density of bright excitations in both subsystems. On the other hand in the over-critical region $\mu v>1$ the subsystem described by the primary field variables remains being of bright-excitation type while the subsystem related to the intermediate field variables converts into the subsystem of dark-excitation type. In the very critical point $\mu \nu=1$ the whole system shrinks into a single subsystem of brightexcitation type.

It is necessary to note that the terms "bright nonlinear excitations" and "dark nonlinear excitations" used in this paper should be understood by analogy with the terms "bright solitons" and "dark solitons" typical of the nonlinear optics [27]. 


\section{Fundamental Poisson brackets for each variant of primary-intermediate field variables [70]}

Now it is time to confirm that the rigorous separation into two subsystems claimed above is maintained also by the key features of fundamental Poisson brackets associated with each particular variant of primary-intermediate field variables. We omit here all calculations based on the formulas for the Poisson brackets between the primary fields (8.18)-(8.32) and on the formulas for the fundamental Poisson brackets between the intermediate fields (10.13)-(10.18) as well as on the auxiliary and transformation formulas listed in the previous Section. Therefore let us consider only the final results.

Thus the first variant of primary-intermediate field variables $q_{+}(n), r_{+}(n), \mathrm{u}_{-}(n), \mathrm{v}_{-}(n)$ is characterized by the following fundamental Poisson brackets

$$
\begin{gathered}
\left\{q_{+}(m), r_{+}(n)\right\}=\mathrm{i}\left[1+q_{+}(n) r_{+}(n)\right] \delta_{n m} \\
\left\{q_{+}(m), q_{+}(n)\right\}=0=\left\{r_{+}(m), r_{+}(n)\right\} \\
\left\{q_{+}(m), \mathrm{v}_{-}(n)\right\}=0=\left\{q_{+}(m), \mathrm{u}_{-}(n)\right\} \\
\left\{\mathrm{u}_{-}(m), r_{+}(n)\right\}=0=\left\{\mathrm{v}_{-}(m), r_{+}(n)\right\} \\
\left\{\mathrm{u}_{-}(m), \mathrm{u}_{-}(n)\right\}=0=\left\{\mathrm{v}_{-}(m), \mathrm{v}_{-}(n)\right\} \\
\left\{\mathrm{u}_{-}(m), \mathrm{v}_{-}(n)\right\}=\frac{\mathrm{i}}{1-\mu \nu}\left[1+\mathrm{u}_{-}(n) \mathrm{v}_{-}(n)\right]\left[1+\mu v \mathrm{u}_{-}(n) \mathrm{v}_{-}(n)\right] \delta_{n m} .
\end{gathered}
$$

Similarly the fundamental Poisson brackets for the second variant of primary-intermediate field variables $q_{-}(n), r_{-}(n), \mathrm{u}_{+}(n), \mathrm{v}_{+}(n)$ are given by the expressions

$$
\begin{gathered}
\left\{q_{-}(m), r_{-}(n)\right\}=\mathrm{i}\left[1+q_{-}(n) r_{-}(n)\right] \delta_{n m} \\
\left\{q_{-}(m), q_{-}(n)\right\}=0=\left\{r_{-}(m), r_{-}(n)\right\} \\
\left\{q_{-}(m), \mathrm{v}_{+}(n)\right\}=0=\left\{q_{-}(m), \mathrm{u}_{+}(n)\right\} \\
\left\{\mathrm{u}_{+}(m), r_{-}(n)\right\}=0=\left\{\mathrm{v}_{+}(m), r_{-}(n)\right\} \\
\left\{\mathrm{u}_{+}(m), \mathrm{u}_{+}(n)\right\}=0=\left\{\mathrm{v}_{+}(m), \mathrm{v}_{+}(n)\right\} \\
\left\{\mathrm{u}_{+}(m), \mathrm{v}_{+}(n)\right\}=\frac{\mathrm{i}}{1-\mu \nu}\left[1+\mathrm{u}_{+}(n) \mathrm{v}_{+}(n)\right]\left[1+\mu v \mathrm{u}_{+}(n) \mathrm{v}_{+}(n)\right] \delta_{n m} .
\end{gathered}
$$

Each of the two above collections (12.1)-(12.6) and (12.7)-(12.12) of fundamental Poisson brackets strictly demonstrates the complete separation between the subsystems of distinct origin.

The accumulated results concerning the separation of field variables and the experience in canonization of other semi-discrete nonlinear systems $[31,54,55,58]$ are sufficient to formulate the nonlinear transformations canonizing the integrable nonlinear system under study (1.1)-(1.6). 


\section{Two variants of canonical field variables $[70,71]$}

The problem of system canonization implies that we have to find out such a set of unified field variables ${ }_{c} \mathrm{y}_{1}(n), \quad c \mathrm{y}_{2}(n),{ }_{c} \mathrm{y}_{3}(n),{ }_{c} \mathrm{y}_{4}(n)$ that the only nonzero elements of structure matrix appearing in the Hamiltonian equations (8.1) to be given by the relations ${ }_{c} \mathrm{~J}_{13}(n \mid m)=-\mathbf{i} \delta_{n m},{ }_{c} \mathbf{J}_{24}(n \mid m)=-\mathbf{i} \delta_{n m}$, ${ }_{c} \mathrm{~J}_{31}(n \mid m)=+\mathrm{i} \delta_{n m},{ }_{c} \mathrm{~J}_{42}(n \mid m)=+\mathrm{i} \delta_{n m}$. Taking into account the fact of system exact splittability into the primary and intermediate subsystems it is reasonable to handle the final step of canonization procedure as the proper nonlinear scaling transformations of primary-intermediate field variables.

Considering that the quantities $\ln \left[1+q_{+}(n) r_{+}(n)\right]$ and $\ln \left[1+\mathrm{u}_{-}(n) \mathrm{v}_{-}(n)\right]-\ln \left[1+\mu v \mathrm{u}_{-}(n) \mathrm{v}_{-}(n)\right]$ taken in under-critical region $\mu \nu<1$ are associated with the densities of excitations respectively in plus-labeled primary subsystem and minus-labeled intermediate subsystem let us introduce the new (physically corrected) field amplitudes $Q_{+}(n), R_{+}(n)$ and $U_{-}(n), V_{-}(n)$ by means of following transformation formulas

$$
\begin{aligned}
& Q_{+}(n)=q_{+}(n) \sqrt{\frac{\ln \left[1+q_{+}(n) r_{+}(n)\right]}{q_{+}(n) r_{+}(n)}} \\
& R_{+}(n)=r_{+}(n) \sqrt{\frac{\ln \left[1+q_{+}(n) r_{+}(n)\right]}{q_{+}(n) r_{+}(n)}}
\end{aligned}
$$

and

$$
\begin{gathered}
U_{-}(n)=\frac{\mathrm{u}_{-}(n)}{\sqrt{\mathrm{u}_{-}(n) \mathrm{v}_{-}(n)}} \sqrt{\ln \frac{1+\mathrm{u}_{-}(n) \mathrm{v}_{-}(n)}{1+\mu \nu \mathrm{u}_{-}(n) \mathrm{v}_{-}(n)}} \\
V_{-}(n)=\frac{\mathrm{v}_{-}(n)}{\sqrt{\mathrm{u}_{-}(n) \mathrm{v}_{-}(n)}} \sqrt{\ln \frac{1+\mathrm{u}_{-}(n) \mathrm{v}_{-}(n)}{1+\mu \nu \mathrm{u}_{-}(n) \mathrm{v}_{-}(n)}} .
\end{gathered}
$$

Here the range of values for the quantity $\left.Q_{+}(n)\right) R_{+}(n)$ is seen to be restricted by the inequality $Q_{+}(n) R_{+}(n) \geq 0$ at any admissible value of background parameter $\mu v$. In contrast the range of values for the quantity $U_{-}(n) V_{-}(n)$ is restricted by the chain of inequalities $\ln (1 / \mu v) \geq U_{-}(n) V_{-}(n) \geq 0$ if $\mu \nu<1$ and by the chain of inequalities $0 \geq U_{-}(n) V_{-}(n) \geq \ln (1 / \mu \nu)$ if $\mu \nu>1$. For the practical purposes the right-hand-side parts of last two expressions (13.3) and (13.4) can be rewritten directly in terms of primary field variables and we obtain

$$
\begin{aligned}
& U_{-}(n)=\frac{q_{-}(n)+\mu(n) q_{+}(n)}{\sqrt{\left[q_{-}(n)+\mu(n) q_{+}(n)\right]\left[r_{-}(n)+v(n) r_{+}(n)\right]}} \sqrt{\ln \frac{1+\mu(n) v(n)+q_{+}(n) r_{+}(n)+q_{-}(n) r_{-}(n)}{(1+\mu v)\left[1+q_{+}(n) r_{+}(n)\right]}} \\
& V_{-}(n)=\frac{r_{-}(n)+v(n) r_{+}(n)}{\sqrt{\left[q_{-}(n)+\mu(n) q_{+}(n)\right]\left[r_{-}(n)+v(n) r_{+}(n)\right]}} \sqrt{\ln \frac{1+\mu(n) v(n)+q_{+}(n) r_{+}(n)+q_{-}(n) r_{-}(n)}{(1+\mu v)\left[1+q_{+}(n) r_{+}(n)\right]}} .
\end{aligned}
$$

The fundamental Poisson brackets related to the field variables $Q_{+}(n), R_{+}(n)$ and $U_{-}(n), V_{-}(n)$ were calculated to be the canonical ones

$$
\left\{Q_{+}(m), R_{+}(n)\right\}=\mathrm{i} \delta_{n m}
$$




$$
\begin{gathered}
\left\{Q_{+}(m), Q_{+}(n)\right\}=0=\left\{R_{+}(m), R_{+}(n)\right\} \\
\left\{Q_{+}(m), V_{-}(n)\right\}=0=\left\{Q_{+}(m), U_{-}(n)\right\} \\
\left\{U_{-}(m), R_{+}(n)\right\}=0=\left\{V_{-}(m), R_{+}(n)\right\} \\
\left\{U_{-}(m), U_{-}(n)\right\}=0=\left\{V_{-}(m), V_{-}(n)\right\} \\
\left\{U_{-}(m), V_{-}(n)\right\}=\mathrm{i} \delta_{n m} .
\end{gathered}
$$

Due to the properties $Q_{+}(n) R_{+}(n) \geq 0, \ln (1 / \mu \nu) \geq U_{-}(n) V_{-}(n) \geq 0$ valid at $\mu \nu<1$ and the properties $Q_{+}(n) R_{+}(n) \geq 0,0 \geq U_{-}(n) V_{-}(n) \geq \ln (1 / \mu v)$ valid at $\mu v>1$ the canonical subsystem described by the field amplitudes $Q_{+}(n)$ and $R_{+}(n)$ can be referred to as the strong one while the canonical subsystem described by the field amplitudes $U_{-}(n)$ and $V_{-}(n)$ can be referred to as the weak one. The strong subsystem corresponds to the bright nonlinear excitations at all admissible values $\mu \nu \geq 0$ of background parameter $\mu \nu$. In contrast the weak subsystem undergoes the crossover from bright-excitation type to dark-excitation type when the background parameter $\mu v$ passes through the critical point $\mu \nu=1$ from its under-critical $\mu \nu<1$ to its over-critical $\mu \nu>1$ values. In the very critical point $\mu \nu=1$ the subsystem of weak excitations persists to be absolutely unexcited. The mutual symmetry between the strong and weak subsystems is seen to be essentially broken at all nonzero values of background parameter and only at $\mu \nu=0$ we come to the completely symmetric situation tantamount to that already being considered earlier [58,64].

The above variant of canonization is originated from the fields variables $q_{+}(n), r_{+}(n)$ and $\mathrm{u}_{-}(n)$, $\mathrm{v}_{-}(n)$, where the intermediate subsystem is labeled by subindex - . For this reason we call it as the minus-asymmetric canonization.

On the other hand the variant of canonization originated from the fields variables $q_{-}(n), r_{-}(n)$ and $\mathrm{u}_{+}(n), \mathrm{v}_{+}(n)$ will be referred to as the the plus-asymmetric one.

The transformation formulas responsible for the plus-asymmetric canonization read as follows

$$
\begin{aligned}
& Q_{-}(n)=q_{-}(n) \sqrt{\frac{\ln \left[1+q_{-}(n) r_{-}(n)\right]}{q_{-}(n) r_{-}(n)}} \\
& R_{-}(n)=r_{-}(n) \sqrt{\frac{\ln \left[1+q_{-}(n) r_{-}(n)\right]}{q_{-}(n) r_{-}(n)}}
\end{aligned}
$$

and

$$
\begin{gathered}
U_{+}(n)=\frac{\mathrm{u}_{+}(n)}{\sqrt{\mathrm{u}_{+}(n) \mathrm{v}_{+}(n)}} \sqrt{\ln \frac{1+\mathrm{u}_{+}(n) \mathrm{v}_{+}(n)}{1+\mu \nu \mathrm{u}_{+}(n) \mathrm{v}_{+}(n)}} \\
V_{+}(n)=\frac{\mathrm{v}_{+}(n)}{\sqrt{\mathrm{u}_{+}(n) \mathrm{v}_{+}(n)}} \sqrt{\ln \frac{1+\mathrm{u}_{+}(n) \mathrm{v}_{+}(n)}{1+\mu \nu \mathrm{u}_{+}(n) \mathrm{v}_{+}(n)}} \\
\text { Co-published by Atlantis Press and Taylor \& Francis } \\
\text { Copyright: the authors } \\
290
\end{gathered}
$$


with the practical versions of last two expressions (13.15) and (13.16) given by formulas

$$
\begin{aligned}
& U_{+}(n)=\frac{q_{+}(n)+v(n) q_{-}(n)}{\sqrt{\left[q_{+}(n)+v(n) q_{-}(n)\right]\left[r_{+}(n)+\mu(n) r_{-}(n)\right]}} \sqrt{\ln \frac{1+\mu(n) v(n)+q_{+}(n) r_{+}(n)+q_{-}(n) r_{-}(n)}{(1+\mu v)\left[1+q_{-}(n) r_{-}(n)\right]}} \\
& V_{+}(n)=\frac{r_{+}(n)+\mu(n) r_{-}(n)}{\sqrt{\left[q_{+}(n)+v(n) q_{-}(n)\right]\left[r_{+}(n)+\mu(n) r_{-}(n)\right]}} \sqrt{\ln \frac{1+\mu(n) v(n)+q_{+}(n) r_{+}(n)+q_{-}(n) r_{-}(n)}{(1+\mu v)\left[1+q_{-}(n) r_{-}(n)\right]}} .
\end{aligned}
$$

The range of values for the quantity $\left.Q_{-}(n)\right) R_{-}(n)$ is restricted by the inequality $Q_{-}(n) R_{-}(n) \geq 0$ at any admissible value of background parameter $\mu v$ while the range of values for the quantity $U_{+}(n) V_{+}(n)$ is restricted by the chain of inequalities $\ln (1 / \mu \nu) \geq U_{+}(n) V_{+}(n) \geq 0$ if $\mu \nu<1$ and by the chain of inequalities $0 \geq U_{+}(n) V_{+}(n) \geq \ln (1 / \mu \nu)$ if $\mu \nu>1$.

As to the fundamental Poisson brackets

$$
\begin{gathered}
\left\{Q_{-}(m), R_{-}(n)\right\}=\mathrm{i} \delta_{n m} \\
\left\{Q_{-}(m), Q_{-}(n)\right\}=0=\left\{R_{-}(m), R_{-}(n)\right\} \\
\left\{Q_{-}(m), V_{+}(n)\right\}=0=\left\{Q_{-}(m), U_{+}(n)\right\} \\
\left\{U_{+}(m), R_{-}(n)\right\}=0=\left\{V_{+}(m), R_{-}(n)\right\} \\
\left\{U_{+}(m), U_{+}(n)\right\}=0=\left\{V_{+}(m), V_{+}(n)\right\} \\
\left\{U_{+}(m), V_{+}(n)\right\}=\mathrm{i} \delta_{n m}
\end{gathered}
$$

related to the field variables $Q_{-}(n), R_{-}(n)$ and $U_{+}(n), V_{+}(n)$ they are seen to be the canonical ones.

In view of the evident mutatis mutandis correspondence with the minus-asymmetric case we do not repeat here all arguments concerning the subdivision of plus-asymmetrically canonized system into the respective strong and weak subsystems and do not explain the details of crossover effect once again.

\section{System Hamiltonian formulations in terms of symmetry-broken canonical field variables [69-71]}

In order to reformulate the integrable nonlinear system under study (1.1)-(1.6) to its canonical form we have to know the the expressions for the primary field variables $q_{+}(n), r_{+}(n), q_{-}(n), r_{-}(n), \mu(n)$, $v(n)$ given in terms of canonical ones $Q_{+}(n), R_{+}(n), U_{-}(n), V_{-}(n)$ or $Q_{-}(n), R_{-}(n), U_{+}(n), V_{+}(n)$.

We begin our consideration with the case of minus-asymmetric canonical fields $Q_{+}(n), R_{+}(n)$ and $U_{-}(n), V_{-}(n)$. Thus inverting the transformation formulas (13.1)-(13.4) we can readily write the primary-intermediate field variables $q_{+}(n), r_{+}(n)$ and $\mathrm{u}_{-}(n), \mathrm{v}_{-}(n)$ in terms of minus-asymmetric canonical ones $Q_{+}(n), R_{+}(n)$ and $U_{-}(n), V_{-}(n)$ respectively. The obtained results should be inserted 
into the transformation formulas (11.3)-(11.6) for the truncated collection of primary fields $q_{-}(n)$, $r_{-}(n), \mu(n), v(n)$. As a consequence we come to the following transformation formulas

$$
\begin{aligned}
& q_{+}(n)=Q_{+}(n) \sqrt{\frac{\exp \left[+Q_{+}(n) R_{+}(n)\right]-1}{Q_{+}(n) R_{+}(n)}} \\
& r_{+}(n)=R_{+}(n) \sqrt{\frac{\exp \left[+Q_{+}(n) R_{+}(n)\right]-1}{Q_{+}(n) R_{+}(n)}}
\end{aligned}
$$

and

$$
\begin{aligned}
& q_{-}(n)=\frac{U_{-}(n)}{\sqrt{U_{-}(n) V_{-}(n)}} \sqrt{\left.\left\{1-\mu v \exp \left[+U_{-}(n) V_{-}(n)\right]\right\} \exp \left[+U_{-}(n) V_{-}(n)\right]-1\right\}}- \\
& -\mu Q_{+}(n) \exp \left[+U_{-}(n) V_{-}(n)\right] \sqrt{\frac{\exp \left[+Q_{+}(n) R_{+}(n)\right]-1}{Q_{+}(n) R_{+}(n)}} \\
& r_{-}(n)=\frac{V_{-}(n)}{\sqrt{U_{-}(n) V_{-}(n)}} \sqrt{\left\{1-\mu v \exp \left[+U_{-}(n) V_{-}(n)\right]\right\}\left\{\exp \left[+U_{-}(n) V_{-}(n)\right]-1\right\}}- \\
& -v R_{+}(n) \exp \left[+U_{-}(n) V_{-}(n)\right] \sqrt{\frac{\exp \left[+Q_{+}(n) R_{+}(n)\right]-1}{Q_{+}(n) R_{+}(n)}} \\
& \mu(n)=\mu \exp \left[+U_{-}(n) V_{-}(n)\right]+\frac{R_{+}(n) U_{-}(n)}{\sqrt{Q_{+}(n) R_{+}(n) U_{-}(n) V_{-}(n)}} \times \\
& \quad \times \sqrt{\left\{1-\mu v \exp \left[+U_{-}(n) V_{-}(n)\right]\right\}\left\{\exp \left[+U_{-}(n) V_{-}(n)\right]-1\right\}\left\{\exp \left[+Q_{+}(n) R_{+}(n)\right]-1\right\}} \\
& v(n)=v \exp \left[+U_{-}(n) V_{-}(n)\right]+\frac{Q_{+}(n) V_{-}(n)}{\sqrt{Q_{+}(n) R_{+}(n) U_{-}(n) V_{-}(n)}} \times \\
& \quad \times \sqrt{\left\{1-\mu \nu \exp \left[+U_{-}(n) V_{-}(n)\right]\right\}\left\{\exp \left[+U_{-}(n) V_{-}(n)\right]-1\right\}\left\{\exp \left[+Q_{+}(n) R_{+}(n)\right]-1\right\}} .
\end{aligned}
$$

The similar reasoning in the case of plus-asymmetric canonical fields $Q_{-}(n), R_{-}(n)$ and $U_{+}(n)$, $V_{+}(n)$ yields

$$
\begin{aligned}
& q_{-}(n)=Q_{-}(n) \sqrt{\frac{\exp \left[Q_{-}(n) R_{-}(n)\right]-1}{Q_{-}(n) R_{-}(n)}} \\
& r_{-}(n)=R_{-}(n) \sqrt{\frac{\exp \left[Q_{-}(n) R_{-}(n)\right]-1}{Q_{-}(n) R_{-}(n)}}
\end{aligned}
$$

and

$$
\begin{aligned}
q_{+}(n) & =\frac{U_{+}(n)}{\sqrt{U_{+}(n) V_{+}(n)}} \sqrt{\left\{1-\mu \nu \exp \left[+U_{+}(n) V_{+}(n)\right]\right\}\left\{\exp \left[+U_{+}(n) V_{+}(n)\right]-1\right\}}- \\
& -v Q_{-}(n) \exp \left[+U_{+}(n) V_{+}(n)\right] \sqrt{\frac{\exp \left[+Q_{-}(n) R_{-}(n)\right]-1}{Q_{-}(n) R_{-}(n)}}
\end{aligned}
$$




$$
\begin{aligned}
& r_{+}(n)=\frac{V_{+}(n)}{\sqrt{U_{+}(n) V_{+}(n)}} \sqrt{\left\{1-\mu v \exp \left[+U_{+}(n) V_{+}(n)\right]\right\}\left\{\exp \left[+U_{+}(n) V_{+}(n)\right]-1\right\}}- \\
& -\mu R_{-}(n) \exp \left[+U_{+}(n) V_{+}(n)\right] \sqrt{\frac{\exp \left[+Q_{-}(n) R_{-}(n)\right]-1}{Q_{-}(n) R_{-}(n)}} \\
& \mu(n)=\mu \exp \left[+U_{+}(n) V_{+}(n)\right]+\frac{Q_{-}(n) V_{+}(n)}{\sqrt{Q_{-}(n) R_{-}(n) U_{+}(n) V_{+}(n)}} \times \\
& \times \sqrt{\left\{1-\mu \nu \exp \left[+U_{+}(n) V_{+}(n)\right]\right\}\left\{\exp \left[+U_{+}(n) V_{+}(n)\right]-1\right\}\left\{\exp \left[+Q_{-}(n) R_{-}(n)\right]-1\right\}} \\
& v(n)=v \exp \left[+U_{+}(n) V_{+}(n)\right]+\frac{R_{-}(n) U_{+}(n)}{\sqrt{Q_{-}(n) R_{-}(n) U_{+}(n) V_{+}(n)}} \times \\
& \times \sqrt{\left\{1-\mu \nu \exp \left[+U_{+}(n) V_{+}(n)\right]\right\}\left\{\exp \left[+U_{+}(n) V_{+}(n)\right]-1\right\}\left\{\exp \left[+Q_{-}(n) R_{-}(n)\right]-1\right\}} .
\end{aligned}
$$

We determine the Hamiltonian functions ${ }_{c} H_{-}$and ${ }_{c} H_{+}$of minus-asymmetrically canonized system and plus-asymmetrically canonized system by substituting the respective transformation formulas (14.1)-(14.6) and (14.7)-(14.12) into the expression (8.6) for the Hamiltonian function ${ }_{p} H$ of primary system (1.1)-(1.6).

Then the dynamics of minus-asymmetrically canonized system will be governed by the Hamiltonian equations

$$
\begin{aligned}
& +\mathrm{i} \dot{Q}_{+}(n)=+\mathrm{i}\left\{{ }_{c} H_{-}, Q_{+}(n)\right\}=\frac{\partial_{c} H_{-}}{\partial R_{+}(n)} \\
& -\mathrm{i} \dot{R}_{+}(n)=-\mathrm{i}\left\{_{c} H_{-}, R_{+}(n)\right\}=\frac{\partial_{c} H_{-}}{\partial Q_{+}(n)} \\
& +\mathrm{i} \dot{U}_{-}(n)=+\mathrm{i}\left\{{ }_{c} H_{-}, U_{-}(n)\right\}=\frac{\partial_{c} H_{-}}{\partial V_{-}(n)} \\
& -\mathrm{i} \dot{V}_{-}(n)=-\mathrm{i}\left\{{ }_{c} H_{-}, V_{-}(n)\right\}=\frac{\partial_{c} H_{-}}{\partial U_{-}(n)}
\end{aligned}
$$

which are seen to have a standard canonical form by virtue of canonical form of the relevant fundamental Poisson brackets (13.7)-(13.12).

Similarly the dynamics of plus-asymmetrically canonized system will be governed by the Hamiltonian equations

$$
\begin{aligned}
& +\mathrm{i} \dot{Q}_{-}(n)=+\mathrm{i}\left\{{ }_{c} H_{+}, Q_{-}(n)\right\}=\frac{\partial_{c} H_{+}}{\partial R_{-}(n)} \\
& -\mathrm{i} \dot{R}_{-}(n)=-\mathrm{i}\left\{{ }_{c} H_{+}, R_{-}(n)\right\}=\frac{\partial_{c} H_{+}}{\partial Q_{-}(n)} \\
& +\mathrm{i} \dot{U}_{+}(n)=+\mathrm{i}\left\{_{c} H_{+}, U_{+}(n)\right\}=\frac{\partial_{c} H_{+}}{\partial V_{+}(n)}
\end{aligned}
$$




$$
-\mathrm{i} \dot{V}_{+}(n)=-\mathrm{i}\left\{{ }_{c} H_{+}, V_{+}(n)\right\}=\frac{\partial_{c} H_{+}}{\partial U_{+}(n)},
$$

where the canonical properties of the relevant fundamental Poisson brackets (13.19)-(13.24) have been taken into account.

\section{Strong and weak standardized components of one-soliton solution [70,71]}

In this Section we illustrate some general results concerning the asymmetric standardizations (13.1)-(13.6) and (13.13)-(13.18) of primary integrable nonlinear system (1.1)-(1.6) on an example of one-soliton solution. For this purpose we shall use the formulas for the one-soliton solution (5.32)-(5.37) of unstandardized system (1.1)-(1.6) given on an infinite ribbon of triangular lattice and characterized by the attractive-type nonlinearities. In so doing we shall omit the upper index + labeling the components of one-soliton solution for the brevity sake. The particular attention in our consideration will be paid to the definitions (5.38)-(5.41) and (5.42) of key one-soliton parameters $\gamma_{+}, x_{+}, \gamma_{-}, x_{-}$and $s$.

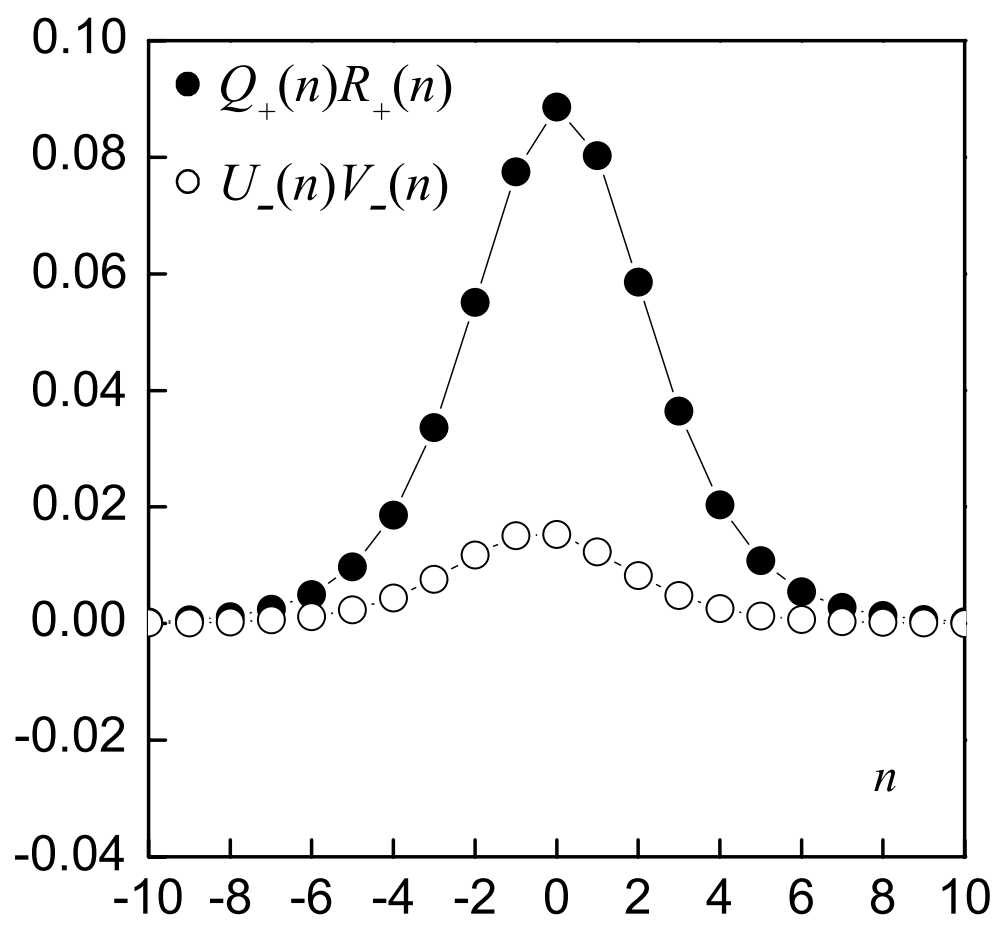

Fig. 6. The typical partitioning of strong $Q_{+}(n) R_{+}(n)$ (full circles) and weak $U_{-}(n) V_{-}(n)$ (empty circles) one-soliton components over the unit cell number $n$ for the case of minus-asymmetric standardization in the under-critical region $\mu \nu<1$ of background parameter $\mu \nu$. The calculations have been made at $\mu=0.7=v, \gamma=0.15, x=0, x=0$ according to formulas (15.2) and (15.3). Both soliton components are seen to be of bright-excitation type. 


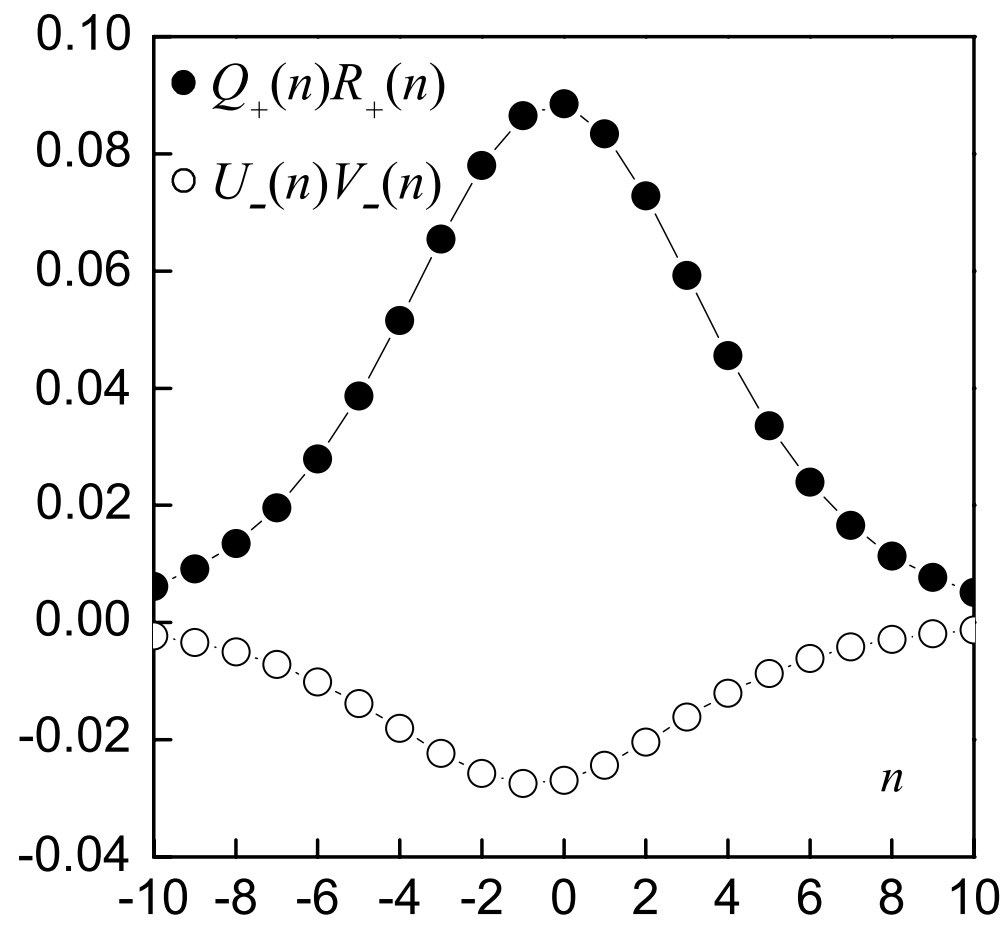

Fig. 7. The typical partitioning of strong $Q_{+}(n) R_{+}(n)$ (full circles) and weak $U_{-}(n) V_{-}(n)$ (empty circles) one-soliton components over the unit cell number $n$ for the case of minus-asymmetric standardization in the over-critical region $\mu \nu>1$ of background parameter $\mu \nu$. The calculations have been made at $\mu=1.9=v, \gamma=0.15, \varkappa=0, x=0$ according to formulas (15.2) and (15.3). The weak soliton component is seen to be of dark-excitation type.

Relying upon the definitions (5.38)-(5.41) of parameters $\gamma_{+}, \varkappa_{+}$and $\gamma_{-}, \varkappa_{-}$one can readily obtain the following expression

$$
\begin{aligned}
\sinh \left[2 \left(\gamma_{+}\right.\right. & \left.\left.+\gamma_{-}-\gamma\right)\right]=(1-\mu v) \frac{\sinh (2 \gamma)}{\cosh \left[2\left(\gamma_{+}+\gamma_{-}-\gamma\right)\right]} \times \\
& \times \frac{2 \sinh (2 \gamma) \sinh (2 \gamma)+[\exp (+2 \mathrm{i} \varkappa)+\mu][\exp (-2 \mathrm{i} \varkappa)+v]}{[\exp (+2 \gamma+2 \mathrm{i} \varkappa)+\mu][\exp (+2 \gamma-2 \mathrm{i} \varkappa)+v][\exp (-2 \gamma+2 \mathrm{i} \varkappa)+\mu][\exp (-2 \gamma-2 \mathrm{i} \varkappa)+v]}
\end{aligned}
$$

indicating that the sign of its left-hand-side term $\sinh \left[2\left(\gamma_{+}+\gamma_{-}-\gamma\right)\right]$ is completely determined by the sign of right-hand-side combination $(1-\mu \nu) \sinh (2 \gamma)$. In particular due to this property the concomitant one-soliton components (5.34) and (5.37) when calculated in the critical point $\mu \nu=1$ are reduced to their limiting constant values $\mu$ and $v$. The same property of the term $\sinh \left[2\left(\gamma_{+}+\gamma_{-}-\gamma\right)\right]$ will be shown to determine the main characteristics of standardized one-soliton components.

Indeed applying the formulas of minus-asymmetric standardization (13.1), (13.2) and (13.5), (13.6) to the quantities $Q_{+}(n) R_{+}(n)$ and $U_{-}(n) V_{-}(n)$ calculated by means of unstandardized multicomponent one-soliton solution (5.32)-(5.37) we obtain

$$
\begin{gathered}
Q_{+}(n) R_{+}(n)=\ln \left\{1+\frac{\sinh (2 \gamma) \sinh (2 \gamma)}{\cosh \left[2\left(\gamma_{+}+\gamma_{-}\right)(n-x-s)\right] \cosh \left[2\left(\gamma_{+}+\gamma_{-}\right)(n-x-s)\right]}\right\} \\
U_{-}(n) V_{-}(n)=\ln \left\{1+\frac{\sinh (2 \gamma) \sinh \left[2\left(\gamma_{+}+\gamma_{-}-\gamma\right)\right]}{\cosh \left[2\left(\gamma_{+}+\gamma_{-}\right)(n-x-3 s+1)\right] \cosh \left[2\left(\gamma_{+}+\gamma_{-}\right)(n-x+s)\right]}\right\} .
\end{gathered}
$$


Thus in accordance with general theory the quantity $Q_{+}(n) R_{+}(n)$ when being calculated on onesoliton solution acquires the real nonnegative values at all admissible values of background parameter $\mu v$ and hence it can be treated as the number of bright $Q_{+} R_{+}$excitations within $n$-th unit sell. This property qualifies the $Q_{+} R_{+}$soliton component as that belonging to the strong subsystem. In contrast the sign of the quantity $U_{-}(n) V_{-}(n)$ calculated on one-soliton solution is seen to be totally manifested by the sign of parameter $1-\mu \nu$ and hence the quantity $U_{-}(n) V_{-}(n)$ can be treated as the number of bright $U_{-} V_{-}$excitations within $n$-th unit sell only at $\mu v<1$. Moreover in the critical point $\mu \nu=1$ the $U_{-} V_{-}$component of minus-asymmetric soliton is vanished completely. At $\mu \nu>1$ the product $U_{-}(n) V_{-}(n)$ becomes negatively semi-definite and hence the $U_{-} V_{-}$component of minus-asymmetric soliton describes the dark excitations. Consequently the $U_{-} V_{-}$component of the minus-asymmetric soliton exhibits all properties predicted for the weak subsystem. Figures 6 and 7 calculated according to formulas (15.2) and (15.3) of minus-asymmetric standardization illustrate the principal distinction in interplay between two mutually asymmetric one-soliton components at under-critical $\mu \nu<1$ (Fig. 6) and over-critical $\mu \nu>1$ (Fig. 7) values of main background parameter $\mu \nu$.

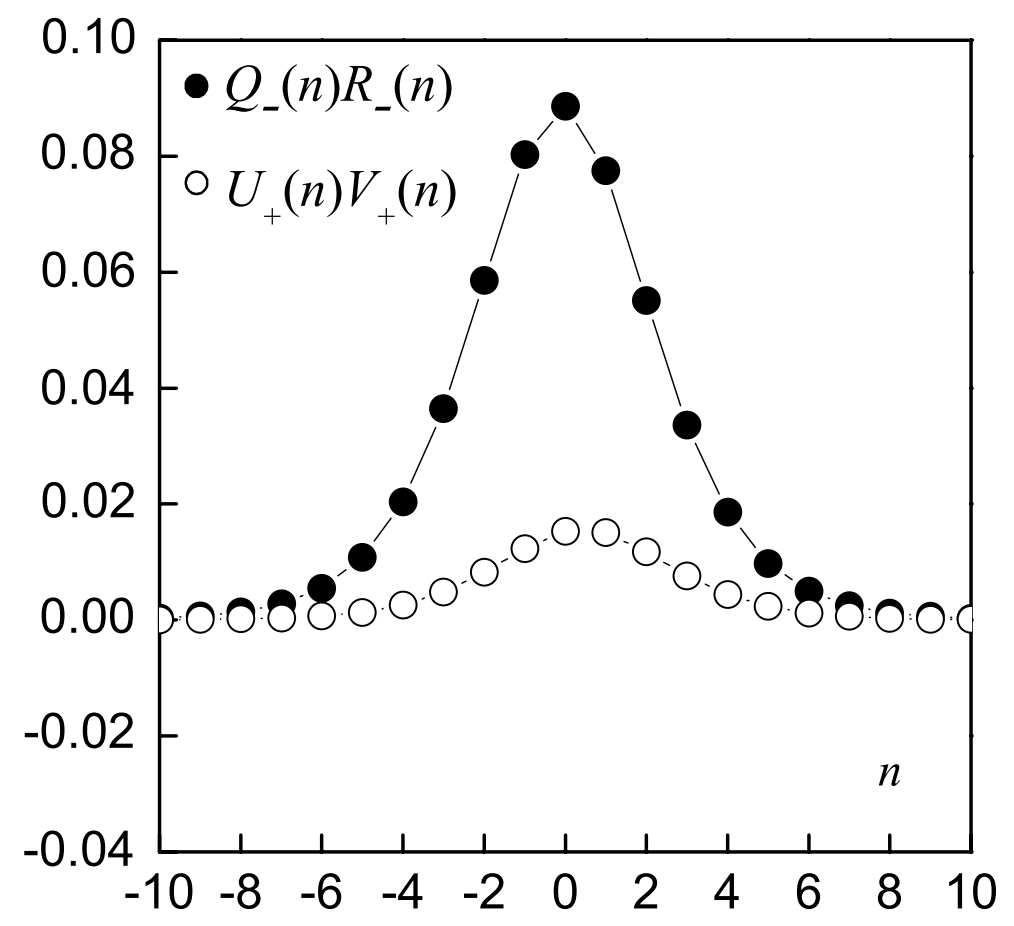

Fig. 8. The typical partitioning of strong $Q_{-}(n) R_{-}(n)$ (full circles) and weak $U_{+}(n) V_{+}(n)$ (empty circles) one-soliton components over the unit cell number $n$ for the case of plus-asymmetric standardization in the under-critical region $\mu \nu<1$ of background parameter $\mu \nu$. The calculations have been made at $\mu=0.7=v, \gamma=0.15, \varkappa=0, x=0$ according to formulas (15.4) and (15.5). Both soliton components are seen to be of bright-excitation type. 


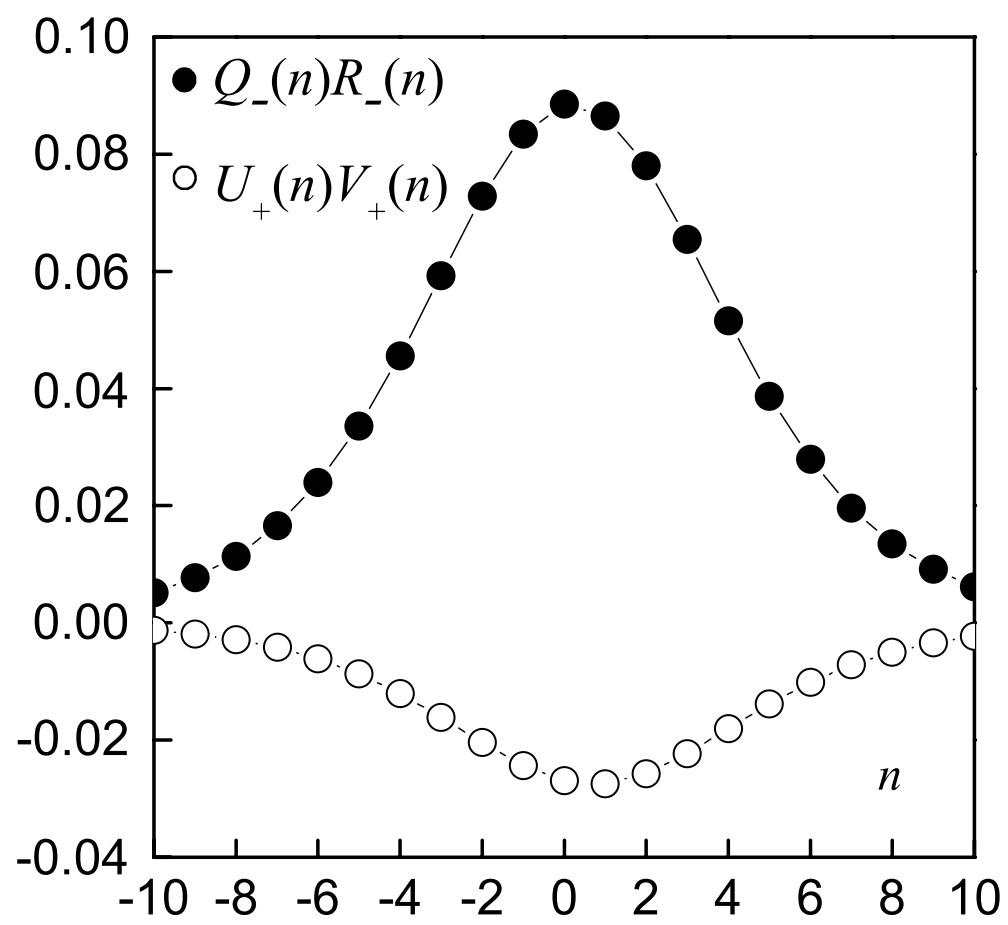

Fig. 9. The typical partitioning of strong $Q_{-}(n) R_{-}(n)$ (full circles) and weak $U_{+}(n) V_{+}(n)$ (empty circles) one-soliton components over the unit cell number $n$ for the case of plus-asymmetric standardization in the over-critical region $\mu \nu>1$ of background parameter $\mu \nu$. The calculations have been made at $\mu=1.9=v, \gamma=0.15, \varkappa=0, x=0$ according to formulas (15.4) and (15.5). The weak soliton component is seen to be of dark-excitation type.

On the other hand applying the formulas of plus-asymmetric standardization (13.13), (13.14) and (13.17), (13.18) to the quantities $Q_{-}(n) R_{-}(n)$ and $U_{+}(n) V_{+}(n)$ calculated by means of unstandardized multi-component one-soliton solution (5.32)-(5.37) we obtain

$$
\begin{gathered}
Q_{-}(n) R_{-}(n)=\ln \left\{1+\frac{\sinh (2 \gamma) \sinh (2 \gamma)}{\cosh \left[2\left(\gamma_{+}+\gamma_{-}\right)(n-x+s)\right] \cosh \left[2\left(\gamma_{+}+\gamma_{-}\right)(n-x+s)\right]}\right\} \\
U_{+}(n) V_{+}(n)=\ln \left\{1+\frac{\sinh (2 \gamma) \sinh \left[2\left(\gamma_{+}+\gamma_{-}-\gamma\right)\right]}{\cosh \left[2\left(\gamma_{+}+\gamma_{-}\right)(n-x+3 s-1)\right] \cosh \left[2\left(\gamma_{+}+\gamma_{-}\right)(n-x-s)\right]}\right\} .
\end{gathered}
$$

Thus in accordance with general theory the quantity $Q_{-}(n) R_{-}(n)$ when being calculated on onesoliton solution acquires the real nonnegative values at all admissible values of background parameter $\mu \nu$ and hence it can be treated as the number of bright $Q_{-} R_{-}$excitations within $n$-th unit sell. This property qualifies the $Q_{-} R_{-}$soliton component as that belonging to the strong subsystem. In contrast the the sign of the quantity $U_{+}(n) V_{+}(n)$ calculated on one-soliton solution is seen to be totally manifested by the sign of parameter $1-\mu v$ and hence the quantity $U_{+}(n) V_{+}(n)$ can be treated as the number of bright $U_{+} V_{+}$excitations within $n$-th unit sell only at $\mu \nu<1$. Moreover in the critical point $\mu \nu=1$ the $U_{+} V_{+}$component of plus-asymmetric soliton is vanished completely. At $\mu v>1$ the product $U_{+}(n) V_{+}(n)$ becomes negatively semi-definite and hence the $U_{+} V_{+}$component of plus-asymmetric soliton describes the dark excitations. Therefore the $U_{+} V_{+}$component of the plus-asymmetric soliton exhibits all properties predicted for the weak subsystem. Figures 8 and 
9 calculated according to formulas (15.4) and (15.5) of plus-asymmetric standardization illustrate the principal distinction in interplay between two mutually asymmetric one-soliton components at under-critical $\mu \nu<1$ (Fig. 8) and over-critical $\mu \nu>1$ (Fig. 9) values of main background parameter $\mu \nu$.

Here we underline that in the critical point $\mu \nu=1$ the parameter of coordinate splitting $s$ (5.42) turns to zero identically by virtue of formula (15.1) for the functional parameter $\sinh \left[2\left(\gamma_{+}+\gamma_{-}-\gamma\right)\right]$. Thus any contradiction between the minus-asymmetric soliton representation and plus-asymmetric soliton representation is absent. The very existence of two nonequivalent subsystems in either of two asymmetrically standardized systems makes it possible to describe the system criticality in the most natural way by eliminating all excitations in a weak subsystem in the critical point.

\section{Conclusion}

In this article we consider the most important properties of the semi-discrete integrable nonlinear Schrödinger system with background-controlled inter-site resonant coupling in view of a significant part that the semi-discrete integrable models of nonlinear Schrödinger type play in the description of different phenomena from various branches of physics. The list of respective references on physical applications can be found in our recent publications [66-68]. The core of reviewed results lies in eight original articles $[62,65-71]$ though the impact of works $[31,54,55]$ dealing with the standardization of famous Ablowitz-Ladik semi-discrete nonlinear system seems to be indispensable. As the matter of fact the author's activity in standardization of Ablowitz-Ladik system [54, 55] has been inspired by rather critical attitude of Academician Alexander S. Davydov towards the nonstandard field amplitudes as those lacking the direct physical sense [63]. The similar problem of standardization as we have already seen concerns also the semi-discrete integrable nonlinear Schrödinger system with background-controlled inter-site resonant coupling however on more sophisticated level [69-71] as compared with the standardization problems appearing in simple semi-discrete integrable nonlinear systems $[50,54,55,58]$ characterized by the splittable structure (symplectic) matrices. On the one hand the splittability of a structure matrix assumes that each of the two diagonal blocks of a structure matrix is a zero matrix, while each of the two off-diagonal blocks of a structure matrix is a diagonal matrix. On the other hand the spittabillity requires that the each element of a structure matrix being given by the field variables belonging to one separate subsystem. There is no universal recipe how to overcome both of above conditions simultaneously. As for the system characterized by the splittable structure matrix the problem of its canonization turns out to be more or less a trivial (though sometimes very cumbersome) task. Thus the main problem in canonization of the semi-discrete integrable nonlinear Schrödinger system with background-controlled inter-site resonant coupling was to find out such a nonlinear transformation to new field variables that the corresponding structure matrix will be splittable. To proceed with this program we were obliged to make a number of logical steps. First of all we have obtained several lowest densities from the infinite hierarchy and established the system Poisson and Hamiltonian structures in terms of primary field variables. Then relying upon the so-called natural constraints we have revealed the system criticality against the background parameter. Taking into account the fact of system criticality we have managed to introduce the set of intermediate field variables and then the two variants of primary-intermediate field variables. Each variant of primary-intermediate field variables is characterized by the the splittable structure matrix and hence the main obstacle for the system canonization 
have been surmounted. In the course of standardization we have discovered that each of standardized system consists of weak subsystem and strong subsystem. The symmetry between the weak subsystem and strong subsystem is essentially broken and can be restored only at zero value of background parameter. In the under-critical region of background parameter both canonical subsystems are the subsystems of bright nonlinear excitations, while in the over-critical region the weak subsystem converts into the subsystem of dark nonlinear excitations. In the very critical point the weak subsystem remains completely unexcited. The crossover in the types of nonlinear excitations has been confirmed by the standardized multi-component one-soliton solution both analytically and graphically with the formulas for the primary (unstandardized) soliton solution being taken into account. The primary soliton solution itself has been obtained in the framework of rather nontrivial Darboux dressing approach developed specially for this purpose.

One more important property of the semi-discrete integrable nonlinear Schrödinger system with background-controlled inter-site resonant coupling is linked with the a priori arbitrary time dependencies of transverse coupling parameters capable to incorporate the effect of external linear potential. As a consequence the primary integrable nonlinear system with appropriately adjusted parametrical driving becomes isomorphic to the system modeling the Bloch oscillations of charged nonlinear carriers in an electrically biased ribbon of triangular lattice. The justification of this statement can be found in our recent paper [68].

\section{Acknowledgments}

The work has been supported by the National Academy of Sciences of Ukraine within the program No 0115U005302. The author is grateful to Vyacheslav O. Vakhnenko for the preparation of Figures $1-9$.

\section{References}

[1] M.J. Ablowitz and J.F. Ladik, Nonlinear differential-difference equations, J. Math. Phys. 16 (1975) 598-603.

[2] M.J. Ablowitz and J.F. Ladik, Nonlinear differential-difference equations and Fourier analysis, J. Math. Phys. 17 (1976) 1011-1018.

[3] M.J. Ablowitz, Y. Ohta and A.D. Trubatch, On discretizations of the vector nonlinear Schrödinger equation, Phys. Lett. A 253 (1999) 287-304.

[4] M.J. Ablowitz, B. Prinari and A.D. Trubatch, Discrete and Continuous Nonlinear Schrödinger Systems (Cambridge University Press, New York, 2004).

[5] M.J. Ablowitz, G. Biondini and B. Prinari, Inverse scattering transform for the integrable discrete nonlinear Schröringer equation with nonvanishing boundary conditions, Inverse Problems 23 (2007) 17111758.

[6] V.E. Adler and V.V. Postnikov, On vector analogs of the modified Volterra lattice, J. Phys. A: Math. Theor. 41 (2008) 455203 (16 pages).

[7] G. Biondini and D. Kraus, Inverse scattering transform for the defocusing Manakov system with nonzero boundary condirions, SIAM J. Math. Anal. 47 (2015) 706-757.

[8] G. Biondini, D.K. Kraus and B. Prinari, The three-component defocusing nonlinear Schrödinger equation with nonzero boundary conditions, Commun. Math. Phys. 348 (2016) 475-533.

[9] L.S. Brizhik, B.M.A.G. Piette and W.J. Zakrzewski, Donor-acceptor electron transport mediated by solitons, Phys. Rev. E 90 (2014) 052915 (12 pages).

[10] R.K. Bullough, N.M. Bogoliubov, A.V. Rybin, G.G. Varzugin and J. Timonen, Solitons of q-deformed quantum lattices and the quantum soliton, J. Phys. A: Math. Gen. 34 (2001) 10463-10474.

[11] P.J. Caudrey, The inverse problem for a general $N \times N$ spectral equation, Physica $D 6$ (1982) 51-66. 
[12] P.J. Caudrey, Differential and discrete spectral problems and their inverses, Wave Phenomena: Modern Theory and Applications, North-Holland Mathematics Studies 97 (Elsevier, Amsterdam, 1984) 221232.

[13] A.R. Chowdhury and G. Mahato, A Darboux-Bäcklund transformation associated with a discrete nonlinear Schödinger equation, Lett. Math. Phys. 7 (1983) 313-317.

[14] D.N. Christodoulides and R.I. Joseph, Discrete self-focusing in nonlinear arrays of coupled waveguides, Opt. Lett. 13 (1988) 794-796.

[15] G. Darboux, Sur le problème de Pfaff, Bull. Sci. Math. Astron. 2 série 6 (1882) 14-36 and 49-68.

[16] A.S. Davydov, Teoriya Molekulyarnykh Eksitonov (Nauka, Moskva, 1968); A.S. Davydov, Theory of Molecular Excitons (Plenum Press, New York-London, 1971).

[17] A.S. Davydov, A.A. Eremko and A.I. Sergienko, Solitony v $\alpha$-spiral'nykh belkovykh molekulakh (Solitons in $\alpha$-helix protein molecules), Ukr. J. Phys. 23 (1978) 983-993.

[18] A.S. Davydov, Solitony v Molekulyarnykh Sistemakh (Naukova Dumka, Kyïv, 1984); A.S. Davydov, Solitons in Molecular Systems (Kluwer Academic, Dordrecht, 1991).

[19] E.V. Doktorov and S.B. Leble, A Dressing Method in Mathematical Physics (Springer, Dordrecht, 2007).

[20] B.A. Dubrovin, S.P. Novikov and A.F. Fomenko, Sovremennaya Geometriya. Metody i Prilozheniya (Nauka, Moskva, 1986);

B.A. Dubrovin, A.F. Fomenko and S.P. Novikov, Modern Geometry. Methods and Applications (Springer-Verlag, Berlin, 1984).

[21] M. Eliashvili, G.I. Japaridze, G. Tsitsishvili and G. Tukhashvili, Edge states in 2D lattices with hopping anisotropy and Chebyshev polynomials, J. Phys. Soc. Japan 83 (2014) 044706 (9 pages).

[22] I.L. Garanovich, S. Longhi, A.A. Sukhorukov and Yu.S. Kivshar, Light propagation and localization in modulated photonic lattices and waveguides, Phys. Rep. 518 (2012) 1-79.

[23] V.S. Gerdzhikov and M.I. Ivanov, Gamil'tonova struktura mnogokomponentnykh raznostnykh nelineynykh uravneniy Shcrödingera, Teor. Mat. Fiz. 52 (1982) 89-104;

V.S. Gerdzhikov and M.I. Ivanov, Hamiltonian structure of multicomponent nonliner Schrödinger equations in difference form, Theor. Math. Phys. 52 (1982) 676-685.

[24] C.H. Gu, H.S. Hu, and Z.X. Zhou, Darboux Transformations in Integrable Systems. Theory and their Applications to Geometry (Kluwer Academic Publishers, Boston-Dordrecht-London, 2005).

[25] L. Jiao, L. Zhang, X. Wang, G. Diankov and H. Dai, Narrow graphene nanoribbons from carbon nanotubes, Nature 458 (2009) 877-880.

[26] F. Kako and N. Mugibayashi, Complete integrability of general nonlinear differential-difference equations solvable by the inverse method. II, Progr. Theor. Phys. 61 (1979) 776-790.

[27] Yu.S. Kivshar and B. Luther-Davies, Dark optical solitons: Physics and applications, Phys. Rep. 298 (1998) 81-197.

[28] K. Konno, H. Sanuki and Y.H. Ichikawa, Conservation laws of nonlinear-evolution equations, Progr. Theor. Phys. 52 (1974) 886-889.

[29] V.V. Konotop, O.A. Chubykalo and L. Vázquez, Dynamics and interaction of solitons on an integrable inhomogeneous lattice, Phys. Rev. E 48 (1993) 563-568.

[30] D.V. Kosynkin, A.L. Higginbotham, A. Sinitskii, J.R. Lomeda, A. Dimiev, B.K. Price and J.M. Tour, Longitudinal unzipping of carbon nanotubes to form graphene nanoribbons, Nature 458 (2009) 872876.

[31] P.P. Kulish, Quantum difference nonlinear Schrödinger equation, Lett. Math. Phys. 5 (1981) 191-197.

[32] V. Kuznetsov and E. Sklyanin, Bäcklund transformation for the BC-type Toda lattice, SIGMA 3 (2007) 080 (17 pages).

[33] S.B. Leble and M.A. Sall', Preobrazovaniye Darbu dlya diskretnogo analoga uravneniy SilinaTikhonchuka, Doklady AN SSSR 284 (1985) 110-114;

S.B. Leble and M.A. Sall', The Darboux transformation for the discrete analog of the Silin-Tikhonchuk equations, Sov. Phys. Doklady 30 (1985) 760-762.

[34] S.B. Leble, Nonlinear Waves and Waveguides with Stratification (Springer-Verlag, Berlin-Heidelberg, 1991). 
[35] R.K.F. Lee, B.J. Cox and J.M. Hill, An exact polyhedral model for boron nanotubes, J. Phys. A: Math. Theor. 42 (2009) 065204 (23 pages).

[36] P. Marquié, J.M. Bilbault and M. Remoissenet, Nonlinear Schrödinger models and modulational instability in real electrical lattices, Physica D 87 (1995) 371-374.

[37] B.M. Maschke, A.J. Van Der Schaft and P.C. Breedveld, An intrinsic Hamiltonian formulation of network dynamics : Non-standard Poisson structures and gyrators, J. Franklin Inst. 329 (1992) 923-966.

[38] V.B. Matveev, Darboux transformation and the explicit solutions of differential-difference and difference-difference evolution equations. I, Lett. Math. Phys. 3 (1979) 217-222.

[39] V.B. Matveev and M.A. Salle, Differential-difference evolution equations. II (Darboux transformation for the Toda lattice), Lett. Math. Phys. 3 (1979) 425-429.

[40] V.B. Matveev and M.A. Salle, Darboux Transformations and Solitons (Springer-Verlag, Berlin - Heidelberg, 1991).

[41] A. Narita, X. Feng, Y. Hernandez, S.A. Jensen, M. Bonn, H. Yang, I.A. Verzhbitskiy, C. Casiraghi, M.R. Hansen, A.H.R. Koch, G. Fytas, O. Ivasenko, B. Li, K.S. Mali, T. Balandina, S. Mahesh, S. De Feyter and K. Müllen, Synthesis of structurally well-defined and liquid-phase-processable graphene nanoribbons, Nature Chemistry 6 (2014) 126-132.

[42] G. Neugebauer and R. Meinel, General $N$-soliton solution of the AKNS class on arbitrary background, Phys. Lett. A 100 (1984) 467-470.

[43] A.C. Newell, Solitons in Mathematics and Physics (SIAM Press, Philadelphia, 1985).

[44] R. Peierls, Zur theorie des diamagnetismus von leitungselektronen, Z. Phys. 80 (1933) 763-791.

[45] A. Rybin, J. Timonen, G. Varzugin and R.K. Bullough, q-deformed solitons and quantum solitons of the Maxwell-Bloch lattice, J. Phys. A: Math. Gen. 34 (2001) 157-164.

[46] R. Scharf and A.R. Bishop, Properties of the nonlinear Schrödinger equation on a lattice, Phys. Rev. A 61 (1991) 6535-6544.

[47] A.C. Scott, Dynamics of Davydov solitons, Phys. Rev. A 26 (1982) 578-595.

[48] V.P. Silin and V.T. Tikhonchuk, Parametricheskaya turbulentnost' i Cherenkovskoye nagrevaniye electronov v prostranstvenno neodnorodnoy plazme, Zh. Eksp. Teor. Fiz. 81 (1981) 2039-2051;

V.P. Silin and V.T. Tikhonchuk, Parametric turbulence and Cherenkov heating of electrons in a spatially inhomogeneous plasma, Sov. Phys. JETP 54 (1981) 1075-1082.

[49] L.A. Takhtadzhyan and L.D. Faddeyev, Gamil'tonov Podkhod v Teorii Solitonov (Nauka, Moskva, 1986);

L.D. Faddeev and L.A. Takhtajan, Hamiltonian Methods in the Theory of Solitons (Springer-Verlag, Berlin, 1987).

[50] Y. Tang, J. Cao, X. Liu and Y. Sun, Symplectic methods for the Ablowitz-Ladik discrete nonlinear Schrödinger equation, J. Phys. A: Math. Theor. 40 (2007) 2425-2437.

[51] T. Tsuchida, H. Ujino and M. Wadati, Integrable semi-discretization of the coupled modified KdV equations, J. Math. Phys. 39 (1998) 4785-4813.

[52] T. Tsuchida, H. Ujino and M. Wadati, Integrable semi-discretization of the coupled nonlinear Schrödinger equations, J. Phys. A: Math. Gen. 32 (1999) 2239-2262.

[53] G.-Z. Tu, A trace identity and its applications to the theory of discrete integrable systems, J. Phys. A: Math. Gen. 23 (1990) 3903-3922.

[54] O.O. Vakhnenko, Nova povnistyu integrovna dyskretyzatsiya neliniynoho rivnyannya Schrödingera (The new comlpletely integrable discretization of the nonlinear Schrödinger equation), Ukr. J. Phys. 40 (1995) 118-122.

[55] O.O. Vakhnenko and V.O. Vakhnenko, Physically corrected Ablowitz-Ladik model and its application to the Peierls-Nabarro problem, Phys. Lett. A 196 (1995) 307-312.

[56] O.O. Vakhnenko, Nonlinear beating excitations on ladder lattice, J. Phys. A: Math. Gen. 32 (1999) 5735-5748.

[57] O.O. Vakhnenko and M.J. Velgakis, Transverse and longitudinal dynamics of nonlinear intramolecular excitations on multileg ladder lattices, Phys. Rev. E 61 (2000) 7110-7120.

[58] O.O. Vakhnenko, Solitons on a zigzag-runged ladder lattice, Phys. Rev. E 64 (2001) 067601 (4 pages). 
[59] O.O. Vakhnenko, Solitons in parametrically driven discrete nonlinear Schrödinger systems with the exploding range of intersite interactions, J. Math. Phys. 43 (2002) 2587-2605.

[60] O.O. Vakhnenko, Three component nonlinear dynamical system generated by the new third-order discrete spectral operator, J. Phys. A: Math. Gen. 36 (2003) 5405-5430.

[61] O.O. Vakhnenko, A discrere nonlinear model of three coupled dynamical fields integrable by the Caudrey method, Ukr. J. Phys. 48 (2003) 653-666.

[62] O.O. Vakhnenko, Integrable nonlinear ladder system with background-controlled intersite resonant coupling, J. Phys. A: Math. Gen. 39 (2006) 11013-1127.

[63] O.O. Vakhnenko, Enigma of probability amplitudes in Hamiltonian formulation of integrable semidiscrete nonlinear Schrödinger systems, Phys. Rev. E 77 (2008) 026604 (9 pages).

[64] O.O. Vakhnenko, Inverse scattering transform for the nonlinear Schrödinger system on a zigzag-runged ladder lattice, J. Math. Phys. 51 (2010) 103518 (45 pages).

[65] O.O. Vakhnenko, Semidiscrete integrable nonlinear systems generated by the new fourth-order spectral operator. Local conservation laws, J. Nonlin. Math. Phys. 18 (2011) 401-414.

[66] O.O. Vakhnenko, Integrable nonlinear Schrödinger system on a triangular-lattice ribbon, J. Phys. Soc. Japan 84 (2015) 014003 (12 pages).

[67] O.O. Vakhnenko, Nonlinear integrable model of Frenkel-like excitations on a ribbon of triangular lattice, J. Math. Phys. 56 (2015) 033505 (21 pages).

[68] O.O. Vakhnenko, Coupling-governed metamorphoses of the integrable nonlinear Schrödinger system on a triangular-lattice ribbon, Phys. Lett. A 380 (2016) 2069-2074.

[69] O.O. Vakhnenko, Asymmetric canonicalization of the integrable nonlinear Schrödinger system on a triangular-lattice ribbon, Appl. Math. Lett. 64 (2017) 81-86.

[70] O.O. Vakhnenko, Symmetry-broken canonizations of the semi-discrete integrable nonlinear Schrödinger system with background-controlled intersite coupling, J. Math. Phys. 57 (2016) 113504 (16 pages).

[71] O.O. Vakhnenko, Distinctive features of the integrable nonlinear Schrödinger system on a ribbon of triangular lattice, Ukr. J. Phys. 62 (2017) xxx-xxx.

[72] V.E. Vekslerchik and V.V. Konotop, Discrete nonlinear Schrödinger equation under non-vanishing boundary conditions, Inverse Problems 8 (1992) 889-909.

[73] M. Wadati, H. Sanuki and K. Konno, Relationships among inverse method, Bäcklund transformation and an infinite number of conservation laws, Progr. Theor. Phys. 53 (1975) 419-436.

[74] X.-Y. Wen, New hierarchies of integrable lattice equations and associated properties: Darboux transformation, conservation laws and integrable coupling, Rep. Math. Phys. 67 (2011) 259-277.

[75] X.-X. Xu, Darboux transformation of a coupled lattice soliton equation, Phys. Lett. A 362 (2007) 205-211.

[76] V.E. Zakharov and E.A. Kuznetsov, Gamil'tonovskiy formalizm dlya nelineynykh voln, Usp. Fiz. Nauk 167 (1997) 1137-1167;

V.E. Zakharov and E.A. Kuznetsov, Hamiltonian formalism for nonlinear waves, Phys.-Uspekhi 40 (1997) 1087-1116.

[77] J.M. Ziman, Models of disorder. The Theoretical Physics of Homogeneously Disordered Systems (Cambridge University Press, Cambridge, 1979). 